\title{
Action and index spectra and periodic orbits in Hamiltonian dynamics
}

\author{
VIKTOR L GINZBURG \\ BAŞAK Z GÜREL
}

\begin{abstract}
The paper focuses on the connection between the existence of infinitely many periodic orbits for a Hamiltonian system and the behavior of its action or index spectrum under iterations. We use the action and index spectra to show that any Hamiltonian diffeomorphism of a closed, rational manifold with zero first Chern class has infinitely many periodic orbits and that, for a general rational manifold, the number of geometrically distinct periodic orbits is bounded from below by the ratio of the minimal Chern number and half of the dimension. These generalizations of the Conley conjecture follow from another result proved here asserting that a Hamiltonian diffeomorphism with a symplectically degenerate maximum on a closed rational manifold has infinitely many periodic orbits.

We also show that for a broad class of manifolds and/or Hamiltonian diffeomorphisms the minimal action-index gap remains bounded for some infinite sequence of iterations and, as a consequence, whenever a Hamiltonian diffeomorphism has finitely many periodic orbits, the actions and mean indices of these orbits must satisfy a certain relation. Furthermore, for Hamiltonian diffeomorphisms of $\mathbb{C P}^{n}$ with exactly $n+1$ periodic orbits a stronger result holds. Namely, for such a Hamiltonian diffeomorphism, the difference of the action and the mean index on a periodic orbit is independent of the orbit, provided that the symplectic structure on $\mathbb{C} \mathbb{P}^{n}$ is normalized to be in the same cohomology class as the first Chern class.
\end{abstract}

\section{Introduction and main results}

\subsection{Introduction}

The main theme of this paper is the interplay between two aspects of the dynamics of Hamiltonian systems: the existence of periodic orbits of arbitrarily large period (or just of infinitely many periodic orbits) and the behavior of the action or (mean) index spectrum under iterations. 
When the manifold is closed and symplectically aspherical, this interplay is fairly unambiguous and can, for instance, be described as follows. On the one hand, as is proved by the authors in [24], the minimal positive action-index gap remains bounded for a certain sequence of iterations, and therefore the action-index spectrum "grows" with iteration. On the other hand, every Hamiltonian system on such a manifold has infinitely many periodic orbits (see Section 1.2) and this fact can easily be inferred from the boundedness of the action-index gap [24]. In fact, all symplectic topological proofs of the existence of infinitely many periodic orbits rely on the analysis of the action or index spectrum; see, eg, Ginzburg [20], Hingston [30], Hofer and Zehnder [34], Salamon and Zehnder [50], Schwarz [52] and Viterbo [56].

However, once the manifold is not assumed to be symplectically aspherical, the question becomes considerably more involved. The first reason for this is that a Hamiltonian diffeomorphism of a closed manifold need not have infinitely many periodic orbits. The second reason is that, since in general the action and index depend on the choice of capping of an orbit, the task of extracting information about the number or growth of the number of orbits from the spectrum becomes much more difficult.

Here we address the following two questions:

- Under what conditions on the manifold and/or the action or index spectrum does a Hamiltonian diffeomorphism have infinitely many (or just many) periodic orbits?

- What are the special features of the action or index spectrum of a Hamiltonian diffeomorphism with only finitely many periodic orbits?

These two questions, although formally equivalent, represent two very different, virtually opposite perspectives focusing on mutually complementary classes of Hamiltonian diffeomorphisms.

The main results of the paper are stated and discussed in detail in Sections 1.2-1.5 and the organization of the paper is outlined in Section 1.6. We refer the reader to Section 2 for necessary definitions and further references.

\subsection{The Conley conjecture}

As we understand it today, the Conley conjecture asserts the existence of infinitely many periodic orbits for any Hamiltonian diffeomorphism $\varphi$ of a closed, symplectically aspherical manifold $M$ or, more precisely, the existence of periodic points of arbitrarily large period, provided that the fixed points are isolated. This conjecture was proved for the so-called weakly nondegenerate Hamiltonian diffeomorphisms by Salamon and 
Zehnder [50] and for all Hamiltonian diffeomorphisms of surfaces other than $S^{2}$ by Franks and Handel [18]. In its original form, as stated by Conley [7] for $M=\mathbb{T}^{2 n}$, the conjecture was established by Hingston [30] and, finally, the case of an arbitrary closed, symplectically aspherical manifold was settled by the first author [20]. (See also, eg, Frauenfelder and Schlenk [19], Gürel [29], Hofer and Zehnder [34], Schwarz [52] and Viterbo [56] for other related results.) The first theorem of this paper, proved in Section 3, is an extension of the Conley conjecture to rational symplectic manifolds with $\left.c_{1}(M)\right|_{\pi_{2}(M)}=0$.

Theorem 1.1 Let $\varphi$ be a Hamiltonian diffeomorphism of a closed, rational symplectic manifold $\left(M^{2 n}, \omega\right)$ with $\left.c_{1}(M)\right|_{\pi_{2}(M)}=0$. Then $\varphi$ has simple periodic orbits of arbitrarily large period whenever the fixed points of $\varphi$ are isolated.

The Conley conjecture obviously fails unless the symplectic manifold (or the diffeomorphism) meets additional requirements such as the condition $\left.c_{1}(M)\right|_{\pi_{2}(M)}=0$ in Theorem 1.1. For instance, the rotation of $S^{2}$ in an irrational angle has only two periodic orbits. (Both of these orbits are fixed points of the rotation.) More generally, we have:

Example 1.2 Let $M$ admit a Hamiltonian torus action with isolated fixed points; see, eg, Cannas da Silva [53], Guillemin, Ginzburg and Karshon [28] and McDuff and Salamon [41] for the definition and further details. Then a generic element of the torus generates a Hamiltonian diffeomorphism of $M$ with finitely many periodic orbits and these orbits are the fixed points of the action. Within this class of manifolds are, for instance, the majority of coadjoint orbits of compact Lie groups. As an explicit example of this type, consider the Hamiltonian $H=\alpha_{0}\left|z_{0}\right|^{2}+\ldots+\alpha_{n}\left|z_{n}\right|^{2}$ on $\mathbb{C} \mathbb{P}^{n}$. Then $H$ generates a circle action with isolated fixed points, provided that the eigenvalues $\alpha_{0}, \ldots, \alpha_{n}$ are rationally independent, ie, linearly independent over $\mathbb{Q}$. Furthermore, when $M$ is as above, the equivariant blow-up of $M$ at the fixed points inherits a Hamiltonian torus action and this action also has, in many instances, isolated fixed points.

Let $N$ denote the minimal Chern number of $M$; see Section 2.1. The following result, also established in Section 3, is of interest when $N$ is large.

Theorem 1.3 Let $M^{2 n}$ be a closed, rational, weakly monotone manifold with $2 N>$ $3 n$. Then any Hamiltonian diffeomorphism $\varphi$ of $M$ has at least $\lceil N / n\rceil$ geometrically distinct periodic orbits. 
For instance, if $\left.c_{1}(M)\right|_{\pi_{2}(M)}=0$, ie, $N=\infty$, the theorem asserts the existence of infinitely many geometrically distinct periodic points, which also follows from Theorem 1.1.

To put Theorem 1.3 in context, note that as has been hypothesized by Michael Chance and Dusa McDuff, the Conley conjecture may hold for closed manifolds with sufficiently many Gromov-Witten invariants equal to zero. For instance, one might expect the Conley conjecture to be true when $N>2 n$ (or $N \geq n$ and $M$ is negative monotone), and hence the quantum product coincides with the cup product; see Example 2.6. Theorems 1.1 and 1.3 can be viewed as a step toward proving this generalization of the Conley conjecture. Note that the manifolds from Example 1.2, for which the Conley conjecture fails, tend to have a large number of nonzero Gromov-Witten invariants (see McDuff [40]) and also have $N \leq n+1$ in all known examples. Among the manifolds satisfying the hypotheses of Theorem 1.1 are Calabi-Yau manifolds. There are also numerous examples of weakly monotone, rational manifolds with large $N<\infty$, though none of these manifolds are monotone; see Example 2.6.

\subsection{The action-index gap}

As was pointed out in Section 1.1, all symplectic geometrical approaches to proving the Conley conjecture type results, such as Theorem 1.1 and Theorem 1.3, rely on the analysis of the sets of actions or indices of periodic orbits (the so-called action and mean index spectra). From this perspective, the Conley conjecture is quite similar to the degenerate case of the Arnold conjecture (see Section 6) and differs significantly from the nondegenerate Arnold conjecture whose proof utilizes a direct count of periodic orbits via Floer homology. Furthermore, extending the proof of the Conley conjecture beyond the case of a symplectically aspherical manifold encounters the same difficulty as the proof of the degenerate Arnold conjecture - differentiating between geometrically distinct orbits and recappings of the same orbit.

The most naive approach to utilizing the action and mean index spectra in proving the Conley conjecture and related results amounts to showing that these spectra, modulo the rationality constant $\lambda_{0}$ or modulo $2 N$, change with iterations. Arguments of this type are discussed in more detail in Section 3. In this section, following our paper [24], we describe a different way of relating the properties of the action and mean index spectra to the dynamics of $\varphi$.

To state the main result, we need to introduce some notation. Let $H$ be a one-periodic in time Hamiltonian on $M$. Then, $H$ can also be viewed as a $k$-periodic Hamiltonian and in this case is denoted by $H^{(k)}$ and referred to as the $k$-th iteration of $H$. Likewise, the $k$-th iteration of a capped periodic orbit $\bar{x}$ is denoted by $\bar{x}^{k}$. The periodic orbits $\bar{x}$ 
and $\bar{y}$ of $H$ are said to be geometrically distinct when the periodic orbits through $x(0)$ and $y(0)$ of the Hamiltonian diffeomorphism $\varphi_{H}$ generated by $H$ are geometrically distinct. Let $\mathcal{A}_{H^{(k)}}(\bar{y})$ and $\Delta_{H^{(k)}}(\bar{y})$ stand for the action and, respectively, the mean index of $H$ on a $k$-periodic orbit $\bar{y}$. We refer the reader to Section 2 for a detailed discussion of these notions. The action gap between two $k$-periodic orbits $\bar{x}$ and $\bar{y}$ of $H$ is then the difference $\mathcal{A}_{H^{(k)}}(\bar{x})-\mathcal{A}_{H^{(k)}}(\bar{y})$ and the mean index gap is defined similarly as the difference $\Delta_{H^{(k)}}(\bar{x})-\Delta_{H^{(k)}}(\bar{y})$. Note that with these definitions, the action or mean index gap can be zero even when $\bar{x}$ and $\bar{y}$ are geometrically distinct. The action-index gap between $\bar{x}$ and $\bar{y}$ is simply the vector in $\mathbb{R}^{2}$ whose components are the action and the mean index gaps. Let also $\|H\|$ denote the Hofer norm of $H$; see Section 2. Finally, recall that an increasing (infinite) sequence of integers $v_{1}<v_{2}<\cdots$ is quasi-arithmetic if $v_{i+1}-v_{i}$ is bounded from above by a constant independent of $i$.

Theorem 1.4 (Bounded gap theorem) Let $H$ be a Hamiltonian on a closed symplectic manifold $\left(M^{2 n}, \omega\right)$ such that all periodic orbits of $\varphi_{H}$ are isolated. Assume that $\left(M^{2 n}, \omega\right)$ is weakly monotone and rational and one of the following conditions holds:

(i) $\|H\|<\lambda_{0}$, where $\lambda_{0}$ is the rationality constant of $M$.

(ii) $N \geq 2 n$.

Then there exists a capped one-periodic orbit $\bar{x}$ of $H$, a quasi-arithmetic sequence of iterations $v_{i}$ and a sequence of capped $v_{i}$-periodic orbits $\bar{y}_{i}$, geometrically distinct from $\bar{x}^{v_{i}}$, such that the sequence of action-index gaps

$$
\left(\mathcal{A}_{H^{\left(v_{i}\right)}}\left(\bar{x}^{v_{i}}\right)-\mathcal{A}_{H^{\left(v_{i}\right)}}\left(\bar{y}_{i}\right), \Delta_{H^{\left(v_{i}\right)}}\left(\bar{x}^{v_{i}}\right)-\Delta_{H^{\left(v_{i}\right)}}\left(\bar{y}_{i}\right)\right)
$$

is bounded.

Corollary 1.5 Let $M$ and $H$ be as in Theorem 1.4. Then there exists a quasiarithmetic sequence of iterations $v_{i}$ and sequences of geometrically distinct $v_{i}$-periodic orbits $\bar{z}_{i}$ and $\bar{z}_{i}^{\prime}$ such that the sequence of action-index gaps between $\bar{z}_{i}$ and $\bar{z}_{i}^{\prime}$ is bounded.

Remark 1.6 The rationality assumption on $M$ can be omitted in (ii) when $H$ is weakly nondegenerate. Furthermore, regarding the role of the condition that all periodic orbits of $H$ are isolated, note that the theorem holds trivially without any assumptions on $M$ or $H$ once this is not the case, ie, $H$ has infinitely many $k$-periodic orbits for some $k$. Observe also that requirement (i) is automatically satisfied when $\lambda_{0}=\infty$, eg, when $M$ is symplectically aspherical. 
Theorem 1.4 is proved in Section 4. When $M$ is symplectically aspherical, the theorem (in a slightly stronger form) was originally established by the authors [24] where it was also observed that in this form the theorem implies the Conley conjecture; cf Hofer and Zehnder [34], Schwarz [52] and Viterbo [56]. This is no longer the case when $M$ is not symplectically aspherical; see Section 4.4. However, Theorem 1.4 and Theorem 1.7 stated below do have an application within the realm of the Conley conjecture, discussed in Section 1.4.

Theorem 1.4 concerns the situation when the quantum aspects of the symplectic topology of $M$ can be neglected. For instance, this is manifested by the fact that, as has been mentioned above, the quantum product coincides with the cup product when $N>2 n$ or by the results from Albers [1], Kerman [36] and Schwarz [51] when $\|H\|<\lambda_{0}$.

In the other extreme case - when certain Gromov-Witten invariants of $M$ are nonzero a much stronger version of Theorem 1.4 holds. Namely, in this case any Hamiltonian $H$ with isolated one-periodic orbits has a nonzero action-index gap bounded by a constant independent of the Hamiltonian. Denote by $\Lambda$ the Novikov ring of $M$, equipped with the valuation $I_{\omega}(A):=-\langle\omega, A\rangle, A \in \pi_{2}(M)$, and by $*$ the product in the quantum homology $\mathrm{HQ}_{*}(M)$; see Section 2.2.1 for the definitions.

Theorem 1.7 (A priori bounded gap theorem) Let $\left(M^{2 n}, \omega\right)$ be a closed, weakly monotone symplectic manifold. Assume that there exists $u \in H_{*<2 n}(M)$ and $w \in$ $H_{*<2 n}(M)$ and $\alpha \in \Lambda$ such that

$$
[M]=(\alpha u) * w .
$$

Let $H$ be a Hamiltonian on $M$ with isolated one-periodic orbits. Assume, in addition, that one of the following conditions holds:

(a) $M$ is rational and $I_{\omega}(\alpha)=\lambda_{0}$.

(b) $2 n-\operatorname{deg} u<2 N$ and $H$ is nondegenerate.

Then $H$ has two geometrically distinct capped one-periodic orbits $\bar{x}$ and $\bar{y}$ such that

$$
\begin{gathered}
0<\mathcal{A}_{H}(\bar{x})-\mathcal{A}_{H}(\bar{y})<I_{\omega}(\alpha), \\
\left\|\Delta_{H}(\bar{x})-n\right\| \leq n \quad \text { and } \quad n \leq\left\|\Delta_{H}(\bar{y})-\operatorname{deg} u\right\| \leq 2 n .
\end{gathered}
$$

Corollary 1.8 Let $M$ and $H$ be as in Theorem 1.7. Then, for every $k$, the Hamiltonian $H$ has two geometrically distinct $k$-periodic orbits $\bar{z}$ and $\bar{z}^{\prime}$ such that the action-index gap between $\bar{z}$ and $\bar{z}^{\prime}$ is nonzero and bounded by a constant independent of $H$ and $k$. 
Proof The orbits $\bar{z}$ and $\bar{z}^{\prime}$ of $H^{(k)}$ are, of course, the orbits $\bar{x}$ and $\bar{y}$ from Theorem 1.7 applied to $H^{(k)}$ treated as a one-periodic Hamiltonian. The fact that $\bar{z}$ and $\bar{z}^{\prime}$ are indeed geometrically distinct readily follows from (1-3) and (1-4); see the proof of Theorem 1.7.

Remark 1.9 The homological condition (1-2) imposes a strong restriction on the symplectic topology of $M$, even without the additional requirement of (a) or (b), and implies, for instance, that $M$ is strongly uniruled in the sense of McDuff [40].

Example 1.10 Let us now list some of the manifolds satisfying the hypotheses of Theorem 1.7; see, eg, McDuff and Salamon [42] and references therein for relevant calculations of the quantum homology.

- The complex projective spaces $\mathbb{C} \mathbb{P}^{n}$ and complex Grassmannians satisfy both (a) and (b); see also Example 2.7 for $\mathrm{HQ}_{*}\left(\mathbb{C P}^{n}\right)$.

- Assume that $M$ satisfies (a) or (b) and $P$ is symplectically aspherical. Then $M \times P$ satisfies (a) or, respectively, (b).

- The product $M \times W$ of two rational manifolds satisfies (a) whenever $M$ does and $\lambda_{0}(W)=m \lambda_{0}(M)$, where $m$ is a positive integer or $\infty$. For instance, (a) holds for the products $\mathbb{C P}^{n} \times \mathbb{C P}^{m_{1}} \times \cdots \mathbb{C P}^{m_{r}}$ with $m_{1}+1, \ldots, m_{r}+1$ divisible by $n+1$ and equally normalized symplectic structures.

- The monotone product $\mathbb{C P}^{n} \times W$, where $\operatorname{gcd}(n+1, N(W)) \geq 2$ and $W$ is monotone, satisfies (b). For instance, the monotone product $\mathbb{C} \mathbb{P}^{n_{1}} \times \cdots \times \mathbb{C P}^{n_{r}}$ meets requirement (b) if $\operatorname{gcd}\left(n_{1}+1, \ldots, n_{r}+1\right) \geq 2$.

- A not-necessarily monotone product $\mathbb{C P}^{1} \times \mathbb{C P}^{1}$ satisfies (b); see Ostrover [46].

Theorem 1.7 is proved in Section 6. Although the statement of the theorem is certainly new, the proof follows a familiar path. On the conceptual level, the argument goes back to the original work of Hofer and Viterbo [33] (see also Liu and Tian [38] and $\mathrm{Lu}$ [39]), where the main underlying principle that the presence of a large space of holomorphic spheres forces the existence of periodic orbits with certain action bounds is established. Our proof of the theorem relies on the technique of action selectors, developed by Hofer and Zehnder [34], Oh [44], Schwarz [52] and Viterbo [56], and on the Hamiltonian version of the Ljusternik-Schnirelman theory (rather than on an explicit use of holomorphic spheres) and bears a resemblance to many an argument found in, eg, Floer [14], Lê and Ono [37] and Schwarz [51] and in Entov and Polterovich [10; 9]. For $M=\mathbb{C P}^{n}$, the theorem (with different action and index bounds) also follows from [10, Section 3]. 


\subsection{Augmented action}

Throughout this section, we assume that $M$ is monotone or negative monotone with monotonicity constant $\lambda<\infty$. Let, as above, $H$ be a one-periodic in time Hamiltonian on $M$ and let $x$ be a one-periodic orbit of $H$. Set

$$
\tilde{\mathcal{A}}_{H}(x)=\mathcal{A}_{H}(\bar{x})-\lambda \Delta_{H}(\bar{x}),
$$

where $\bar{x}$ is the orbit $x$ equipped with an arbitrary capping. It is clear that $\tilde{\mathcal{A}}_{H}(x)$, referred to as the augmented action in what follows, is independent of the capping. (When $\bar{x}$ is a $k$-periodic orbit of a $k$-periodic Hamiltonian $H$, the augmented action is defined in a similar fashion.) This definition is inspired by considerations in Salamon [49, Section 1.6] and Entov and Polterovich [9, Section 1.4], where the Conley-Zehnder index is utilized in place of the mean index. For us, the main advantage of using the mean index is that $\widetilde{\mathcal{A}}_{H}(x)$ is defined even when $x$ is degenerate and that the augmented action is homogeneous, ie,

$$
\tilde{\mathcal{A}}_{H^{(k)}}\left(x^{k}\right)=k \tilde{\mathcal{A}}_{H}(x),
$$

since $\mathcal{A}_{H^{(k)}}\left(\bar{x}^{k}\right)=k \mathcal{A}_{H}(\bar{x})$ and $\Delta_{H^{(k)}}\left(\bar{x}^{k}\right)=k \Delta_{H}(\bar{x})$. Furthermore, when $x$ is a $k$-periodic orbit of a one-periodic Hamiltonian $H$, it is convenient to define the normalized augmented action as $\tilde{\mathcal{A}}_{H}(x)=\widetilde{\mathcal{A}}_{H^{(k)}}(x) / k ;$ cf Section 3.1. Then, by (1-5), iterating an orbit does not change the normalized augmented action: $\widetilde{\mathcal{A}}_{H}\left(x^{k}\right)=\widetilde{\mathcal{A}}_{H}(x)$. Moreover, geometrically identical orbits have equal normalized augmented action.

Corollary 1.11 Let $M$ be monotone or negative monotone with $\lambda<\infty$ and let $M$ and $H$ satisfy the hypotheses of Theorem 1.4 or Theorem 1.7. Assume also that $H$ has finitely many periodic orbits. Then there exist two geometrically distinct periodic orbits $x$ and $y$ such that

$$
\tilde{\mathcal{A}}_{H}(x)=\tilde{\mathcal{A}}_{H}(y) \text {. }
$$

In other words, $H$ has infinitely many periodic orbits, provided that any two geometrically distinct orbits have different normalized augmented actions. The corollary easily follows from Theorem 1.4 and Theorem 1.7. Indeed, arguing by contradiction, assume that $H$ has finitely many periodic orbits and the difference $\widetilde{\mathcal{A}}_{H}(x)-\widetilde{\mathcal{A}}_{H}(y)$ is never zero. Then $\tilde{\mathcal{A}}_{H^{(k)}}\left(x^{k}\right)-\widetilde{\mathcal{A}}_{H^{(k)}}\left(y^{k}\right) \rightarrow \infty$ as $k \rightarrow \infty$ for any pair of geometrically distinct periodic orbits. As a consequence, the minimal action-index gap between geometrically distinct $k$-periodic orbits with arbitrary cappings goes to infinity, which contradicts Corollary 1.5 or 1.8. One essentially immediate consequence of Corollary 1.11 is the generic existence of infinitely many periodic orbits under the hypotheses of the corollary; see Ginzburg and Gürel [23]. 
When $M=\mathbb{C} \mathbb{P}^{n}$, we have the following much more precise result, which is proved in Section 6, along with Theorem 1.7, as a consequence of the Hamiltonian LjusternikSchnirelman theory.

Theorem 1.12 Let $H$ be a Hamiltonian on $\mathbb{C P}^{n}$ with exactly $n+1$ geometrically distinct periodic orbits $x_{0}, \ldots, x_{n}$. Then

$$
\tilde{\mathcal{A}}_{H}\left(x_{0}\right)=\cdots=\tilde{\mathcal{A}}_{H}\left(x_{n}\right) \text {. }
$$

Regarding the assumptions of this theorem, recall that every Hamiltonian on $\mathbb{C} \mathbb{P}^{n}$ has at least $n+1$ one-periodic orbits by the Arnold conjecture for $\mathbb{C} \mathbb{P}^{n}$ proved by Fortune [16] and Fortune and Weinstein [17]; see Section 6 for further discussion. Thus, in the setting of Theorem 1.12, every periodic orbit $x_{i}$ is one-periodic. On the other hand, hypothetically, every Hamiltonian on $\mathbb{C P}^{n}$ with more than $n+1$ oneperiodic orbits necessarily has infinitely many geometrically distinct periodic orbits. This is a variant of the Conley conjecture specific to $\mathbb{C} \mathbb{P}^{n}$. (The case of $\mathbb{C P}^{1}$ is established in Franks and Handel [18].) Thus, if we assume this version of the Conley conjecture, every Hamiltonian on $\mathbb{C} \mathbb{P}^{n}$ with finitely many periodic orbits must satisfy the requirements of Theorem 1.12.

Example 1.13 Let $M$ and $H$ be as in Example 1.2 and let $M$ be monotone. Then $\widetilde{\mathcal{A}}_{H}\left(x_{i}\right)=\widetilde{\mathcal{A}}_{H}\left(x_{j}\right)$ for any two one-periodic orbits of $H$ on $M$. (This fact is not hard to prove using equivariant cohomology and localization theorems; see, eg, Guillemin, Ginzburg and Karshon [28, Appendix C].) For instance, consider the Hamiltonian $H=c+\sum \alpha_{j}\left|z_{j}\right|^{2}$ on $\mathbb{C P}^{n}$ where the eigenvalues $\alpha_{j}$ are rationally independent and $c$ is chosen so that $H$ is normalized: $\int H \omega^{n}=0$. Then, as a direct calculation shows $\tilde{\mathcal{A}}_{H}\left(x_{j}\right)=0$ for all $n+1$ fixed points $x_{j}$.

This example suggests that an analogue of Theorem 1.12 may hold for any Hamiltonian $H$ with finitely many periodic orbits on a monotone manifold or, at least, many (not just two as in Corollary 1.11) of augmented actions $\widetilde{\mathcal{A}}_{H}\left(x_{i}\right)$ should be equal. Another question that naturally arises is what $\widetilde{\mathcal{A}}_{H}\left(x_{i}\right)$ is equal to in the setting of Theorem 1.12. Is $\tilde{\mathcal{A}}_{H}\left(x_{i}\right)=0$, when $H$ is normalized, as it is for normalized quadratic Hamiltonians on $\mathbb{C} \mathbb{P}^{n}$ ?

Remark 1.14 The augmented action carries nearly as much information as the pair $\left(\Delta_{H}, \mathcal{A}_{H}\right)$ when $N<\infty$ and $\lambda<\infty$. To visualize the relation between the two invariants, fix a periodic orbit $\bar{x}$. Consider the sequence of points $\left(\Delta_{H}(\bar{x} \# A), \mathcal{A}_{H}(\bar{x} \# A)\right)$, where $A \in \pi_{2}(M)$, on the $\left(\Delta_{H}, \mathcal{A}_{H}\right)$-plane. This sequence lies on the line with slope $\lambda$, intersecting the $\mathcal{A}_{H}$-axis at $\tilde{\mathcal{A}}_{H}(x)$, and the adjacent points differ by the 
vector $\left(2 N, \lambda_{0}\right)$. Then, the action-index gaps are vectors connecting points from sequences corresponding to geometrically distinct periodic orbits of the same period. Corollary 1.11 asserts that after passing to a sufficiently high iteration of $H$ at least two sequences lie on the same line, when $H$ has finitely many periodic orbits. Furthermore, in the setting of Theorem 1.12, all $n+1$ sequences lie on the same line.

\subsection{Symplectically degenerate maxima}

All of the theorems stated above, with the exception of Theorem 1.7 and Theorem 1.12, rely on the automatic existence of periodic orbits in the presence of symplectically degenerate maxima. This phenomenon was essentially discovered by Hingston [30] and then further investigated and used by the first author in [20] and both authors in [24].

Definition 1.15 An isolated capped periodic orbit $\bar{x}$ of a Hamiltonian $H$ is said to be a symplectically degenerate maximum of $H$ if $\Delta_{H}(\bar{x})=0$ and $\operatorname{HF}_{2 n}(H, \bar{x}) \neq 0$.

Here, $\mathrm{HF}_{*}(H, \bar{x})$ stands for the local Floer homology of $H$ at $\bar{x}$, graded throughout the paper so that a $C^{2}$-small nondegenerate autonomous maximum has degree $2 n$. We refer the reader to [24] for a detailed study of symplectically degenerate maxima and to Section 2.5 for a discussion of the local Floer homology. At this point we only note that in Definition 1.15 the condition that $\Delta_{H}(\bar{x})=0$ can be replaced by the requirement that $x$ be totally degenerate and that $H$ and $\bar{x}$ are assumed to have the same period.

Example 1.16 Let $\bar{x}$ be an isolated totally degenerate maximum, equipped with trivial capping, of an autonomous Hamiltonian. Then $\bar{x}$ is a symplectically degenerate maximum.

The following key result is proved in Section 5 in the present form and in [20] in the case where $M$ is symplectically aspherical.

Theorem 1.17 Assume that $\left(M^{2 n}, \omega\right)$ is weakly monotone and rational, and let $\bar{x}$ be a symplectically degenerate maximum of $H$. Set $c=\mathcal{A}_{H}(\bar{x})$. Then for every sufficiently small $\epsilon>0$ there exists $k_{\epsilon}$ such that

$$
\mathrm{HF}_{2 n+1}^{\left(k c+\delta_{k}, k c+\epsilon\right)}\left(H^{(k)}\right) \neq 0 \text { for all } k>k_{\epsilon} \text { and some } \delta_{k} \text { with } 0<\delta_{k}<\epsilon .
$$

Using Theorem 1.17 , it is not hard to show that $H$ has infinitely many geometrically distinct periodic orbits whenever it has a symplectically degenerate maximum. More precisely, in Section 3, we will prove the following: 
Theorem 1.18 Let $H$ be a one-periodic Hamiltonian on a closed, weakly monotone and rational manifold $M$.

(i) Assume that some iteration $H^{\left(k_{0}\right)}$ has finitely many $k_{0}$-periodic orbits and also has a symplectically degenerate maximum. Then $H$ has infinitely many geometrically distinct periodic orbits.

(ii) If, in addition, $k_{0}=1$ and $\left.\omega\right|_{\pi_{2}(M)}=0$ or $\left.c_{1}(M)\right|_{\pi_{2}(M)}=0$, the Hamiltonian $H$ has simple periodic orbits of arbitrarily large period.

Remark 1.19 Note that the assumption that $M$ is rational plays a purely technical role in the proof of Theorem 1.17 and, perhaps, can be eliminated entirely. This requirement, however, is also used in other ways in the proof of Theorem 1.3 and of Theorem 1.18, except the case where $\left.c_{1}(M)\right|_{\pi_{2}(M)}=0$. In particular, the rationality requirement enters the proof of Theorem 1.1 only via Theorem 1.17. We will further discuss these results in Section 3.

\subsection{Organization of the paper}

The role of the next section, Section 2, is purely technical: here we set conventions and notation and recall relevant results concerning filtered and local Floer homology, quantum homology, homotopy maps, the mean index and loops of Hamiltonian diffeomorphisms. This section is intended for quick reference rather than for "linear reading". The main results of the paper are proved in the following four sections, which are essentially independent of each other. In Section 3, we establish Theorems $1.1,1.3$ and 1.18 , along with some auxiliary results on the action and index spectra. Theorem 1.4 is proved in Section 4, following closely [24]. In Section 5, we prove Theorem 1.17, which the previous results rely on in the case where the Hamiltonian has a symplectically degenerate maximum. This proof utilizes a direct sum decomposition of the filtered Floer homology for small action intervals, which reduces the problem to the case of a symplectically aspherical manifold (or even $\mathbb{R}^{2 n}$ ), treated in [26; $24]$ and is of independent interest. Finally, in Section 6, we discuss the technique of action selectors and the Hamiltonian Ljusternik-Schnirelman theory and, after a brief digression into the degenerate case of the Arnold conjecture, prove Theorem 1.7 and Theorem 1.12.

Acknowledgments The authors are grateful to Doris Hein and Dusa McDuff for useful discussions.

The work is partially supported by the NSF and by the faculty research funds of the University of California, Santa Cruz. 


\section{Preliminaries}

The goal of this section is to set notation and conventions and to give a brief review of Floer and quantum homology and several other notions used in the paper. Most of this material is quite standard and the only new results discussed here concern extensions of loops of Hamiltonian diffeomorphisms; see Section 2.3.

\subsection{Conventions and basic definitions}

The objective of this subsection is to set terminology and conventions and recall basic definitions.

2.1.1 Symplectic manifolds and Hamiltonian flows Let $(M, \omega)$ be a symplectic manifold. Recall that $M$ is said to be monotone (negative monotone) if $\left.[\omega]\right|_{\pi_{2}(M)}=$ $\left.\lambda c_{1}(M)\right|_{\pi_{2}(M)}$ for some nonnegative (respectively, negative) constant $\lambda$ and $M$ is rational if $\left\langle[\omega], \pi_{2}(M)\right\rangle=\lambda_{0} \mathbb{Z}$, ie, the integrals of $\omega$ over spheres in $M$ form a discrete subgroup of $\mathbb{R}$. (When $\left\langle[\omega], \pi_{2}(M)\right\rangle=0$, we set $\lambda_{0}=\infty$.) The constants $\lambda$ and $\lambda_{0} \geq 0$ will be referred to as the monotonicity and rationality constants. The positive generator $N$ of the discrete subgroup $\left\langle c_{1}(M), \pi_{2}(M)\right\rangle \subset \mathbb{R}$ is called the minimal Chern number of $M$. When this subgroup is zero, we set $N=\infty$. The manifold $M$ is called symplectically aspherical if $\left.c_{1}(M)\right|_{\pi_{2}(M)}=0=\left.[\omega]\right|_{\pi_{2}(M)}$. A symplectically aspherical manifold is monotone and a monotone or negative monotone manifold is rational.

To ensure that the standard construction of Floer homology applies, all symplectic manifolds $(M, \omega)$ are required, throughout the paper, to be weakly monotone, ie, monotone or $N \geq n-2$ (including the case $\left.c_{1}(M)\right|_{\pi_{2}(M)}=0$ ). Utilizing the machinery of virtual cycles, one can eliminate this requirement in Theorems 1.1, 1.3, 1.4, 1.17 and 1.18 .

Furthermore, all Hamiltonians $H$ on $M$ considered in this paper are assumed to be $k$-periodic in time, ie, $H: S_{k}^{1} \times M \rightarrow \mathbb{R}$, where $S_{k}^{1}=\mathbb{R} / k \mathbb{Z}$, and the period $k$ is always a positive integer. When the period is not specified, it is equal to one, which is the default period in this paper. We set $H_{t}=H(t, \cdot)$ for $t \in S^{1}=\mathbb{R} / \mathbb{Z}$. The Hamiltonian vector field $X_{H}$ of $H$ is defined by $i_{X_{H}} \omega=-d H$. The (time-dependent) flow of $X_{H}$ will be denoted by $\varphi_{H}^{t}$ and its time-one map by $\varphi_{H}$. Such time-one maps are referred to as Hamiltonian diffeomorphisms. A Hamiltonian $H$ is said to be normalized if $\int_{M} H_{t} \omega^{n}=0$ for all $t \in S^{1}$. Below, in contrast with, say, [52], the Hamiltonians are not by default assumed to be normalized.

A one-periodic Hamiltonian $H$ can always be treated as $k$-periodic. In this case, we will use the notation $H^{(k)}$ and, abusing terminology, call $H^{(k)}$ the $k$-th iteration 
of $H$. Incorporating the period $k$ into the notation is often redundant and awkward, but it does eliminate any ambiguity and is convenient when a Hamiltonian or an orbit can be viewed as $k$-periodic for different periods $k$.

The Hofer norm of a $k$-periodic Hamiltonian $H$ is defined by

$$
\|H\|=\int_{0}^{k}\left(\max _{M} H_{t}-\min _{M} H_{t}\right) d t .
$$

Let $K$ and $H$ be two one-periodic Hamiltonians. The composition $K \# H$ is the Hamiltonian

$$
(K \# H)_{t}=K_{t}+H_{t} \circ\left(\varphi_{K}^{t}\right)^{-1}
$$

generating the flow $\varphi_{K}^{t} \circ \varphi_{H}^{t}$. In general, $K \# H$ is not one-periodic. However, this is the case if, for example, $H_{0} \equiv 0 \equiv H_{1}$. The latter condition can be met by reparametrizing the Hamiltonian as a function of time without changing the time-one map. Thus, in what follows, we will usually treat $K \# H$ as a one-periodic Hamiltonian. Another instance when the composition $K \# H$ of two one-periodic Hamiltonians is automatically oneperiodic is when the flow $\varphi_{K}^{t}$ is a loop of Hamiltonian diffeomorphisms, ie, $\varphi_{K}^{1}=\mathrm{id}$. We set $H^{\# k}=H \# \cdots \# H$ ( $k$ times). The flow $\varphi_{H^{\# k}}^{t}=\left(\varphi_{H}^{t}\right)^{k}, t \in[0,1]$, is homotopic with fixed end-points to the flow $\varphi_{H}^{t}, t \in[0, k]$. Note also that $\left\|H^{(k)}\right\|=k\|H\|=\left\|H^{\# k}\right\|$.

2.1.2 Capped periodic orbits Let $x: S_{k}^{1} \rightarrow W$ be a contractible loop. A capping of $x$ is a map $u: D^{2} \rightarrow M$ such that $\left.u\right|_{S_{k}^{1}} ^{1}=x$. Two cappings $u$ and $v$ of $x$ are considered to be equivalent if the integrals of $\omega$ and $c_{1}(M)$ over the sphere obtained by attaching $u$ to $v$ are both equal to zero. For instance, when $M$ is symplectically aspherical, all cappings of $x$ are equivalent to each other. A capped closed curve $\bar{x}$ is, by definition, a closed curve $x$ equipped with an equivalence class of capping. In what follows, the presence of capping is always indicated by a bar. We denote by $\mathcal{P}(H)$ the collection of all one-periodic orbits of $H$ and by $\overline{\mathcal{P}}(H)$ the collection of its capped one-periodic orbits.

The action of a one-periodic Hamiltonian $H$ on a capped closed curve $\bar{x}=(x, u)$ is defined by

$$
\mathcal{A}_{H}(\bar{x})=-\int_{u} \omega+\int_{S^{1}} H_{t}(x(t)) d t .
$$

The space of capped closed curves is a covering space of the space of contractible loops and the critical points of $\mathcal{A}_{H}$ on the former space are exactly one-periodic orbits of $X_{H}$. The action spectrum $\mathcal{S}(H)$ of $H$ is the set of critical values of $\mathcal{A}_{H}$. This is a zero measure set. When $M$ is rational, $\mathcal{S}(H)$ is a closed, and hence nowhere dense, set. Otherwise, $\mathcal{S}(H)$ is everywhere dense. Furthermore, when $M$ is rational, the action $\mathcal{A}_{H}(\bar{x})$, viewed modulo $\lambda_{0}$, is independent of the capping. We denote by 
$\mathcal{S}_{\lambda_{0}}(H) \subset S_{\lambda_{0}}^{1}=\mathbb{R} / \lambda_{0} \mathbb{Z}$ the action spectrum modulo $\lambda_{0}$. These definitions extend to $k$-periodic orbits and Hamiltonians in an obvious way.

In this paper, we are only concerned with contractible periodic orbits and a periodic orbit is always assumed to be contractible, even if this is not explicitly stated.

A (capped) periodic orbit $\bar{x}$ of $H$ is nondegenerate if the linearized return map $d \varphi_{H}: T_{x(0)} W \rightarrow T_{x(0)} W$ has no eigenvalues equal to one. Following [50], we call $x$ weakly nondegenerate if at least one of the eigenvalues is different from one. Otherwise, the orbit is said to be strongly degenerate. Clearly, capping has no effect on degeneracy or nondegeneracy of $\bar{x}$. A Hamiltonian is nondegenerate if all its one-periodic orbits are nondegenerate.

Let $\bar{x}$ be a nondegenerate (capped) periodic orbit. The Conley-Zehnder index $\mu_{\mathrm{CZ}}(\bar{x}) \in$ $\mathbb{Z}$ is defined, up to a sign, as in $[49 ; 50]$. More specifically, in this paper, the ConleyZehnder index is the negative of that in [49]. In other words, we normalize $\mu_{\mathrm{Cz}}$ so that $\mu_{\mathrm{CZ}}(\bar{x})=n$ when $x$ is a nondegenerate maximum (with trivial capping) of an autonomous Hamiltonian with small Hessian. Sometimes, we will also use the notation $\mu_{\mathrm{CZ}}(H, \bar{x})$.

As is well-known, the fixed points of $\varphi_{H}:=\varphi_{H}^{1}$ are in one-to-one correspondence with (not-necessarily contractible) one-periodic orbits of $H$. Likewise, the $k$-periodic points of $\varphi_{H}$, ie, the fixed points of $\varphi_{H}^{k}$, are in one-to-one correspondence with (notnecessarily contractible) $k$-periodic orbits of $H$. The $k$-th iteration of a one-periodic orbit $x$ of $H$ is the orbit $x(t)$, where $t$ now ranges in [0,k]. It is easy to see that the iteration $\bar{x}^{k}$ of a capped orbit $\bar{x}$ carries a natural capping. The action functional is homogeneous with respect to iteration:

$$
\mathcal{A}_{H^{(k)}}\left(\bar{x}^{k}\right)=k \mathcal{A}_{H}(\bar{x}) .
$$

Two periodic orbits $x$ and $y$ with possibly different periods are said to be geometrically distinct if the sets of points $\varphi_{H}^{i}(x(0))=x(i)$ and $\varphi_{H}^{j}(y(0))=y(j)$ are distinct, ie, if the corresponding periodic orbits of $\varphi_{H}$ are geometrically distinct. Otherwise, we call the orbits geometrically identical or equivalent. These notions extend to capped orbits via forgetting the cappings. We will denote the set of geometrically distinct periodic orbits of $H$ (or, more precisely, the set of geometrical equivalence classes) by $\mathcal{P}^{\infty}(H)$. The set of such orbits of period less than or equal to $k$ will be denoted by $\mathcal{P}_{k}(H)$. It is worth emphasizing that two $k$-periodic orbits $x$ and $y$ of $H$, with $k>1$, can be distinct (ie, $x \neq y$ ) but geometrically equivalent. The capped orbits obtained by capping an orbit $x$ in all possible ways are geometrically identical to each other and an orbit $x$ and its iteration $x^{k}$ are geometrically identical. Two one-periodic orbits $x$ and $y$ are geometrically distinct if and only if $x(0) \neq y(0)$. Let $\bar{x}$ and $\bar{y}$ be $k$-and, 
respectively, $l$-periodic orbits which are geometrically identical and $M$ is rational. Then

$$
\frac{\mathcal{A}_{H^{(k)}}(\bar{x})}{k} \equiv \frac{\mathcal{A}_{H^{(l)}}(\bar{y})}{l} \bmod \lambda_{0} \mathbb{Q} .
$$

The converse, of course, is not true.

There is a natural action and index preserving one-to-one correspondence between capped $k$-periodic orbits of $H$ and capped one-periodic orbits of $H^{\# k}$. Thus, when convenient, we will treat $\bar{x}^{k}$ as a one-periodic orbit of $H^{\# k}$.

\subsection{Floer homology and quantum homology}

In this section, we review the construction and basic properties of Floer homology, mainly to further specify notation and conventions.

Recall that when $M$ is closed and weakly monotone, the filtered Floer homology of $H: S^{1} \times M \rightarrow \mathbb{R}$ for the interval $(a, b)$, denoted throughout the paper by $\mathrm{HF}_{*}^{(a, b)}(H)$, is defined. We refer the reader to Floer's papers $[11 ; 12 ; 13 ; 15]$ or to, eg, [32; 42; 49] for further references and introductory accounts of the construction of (Hamiltonian) Floer homology. The terminology, conventions and most of the notation used here are similar to those in $[22 ; 20 ; 29]$. Note however that now we grade the Floer complex and homology by $\mu_{\mathrm{CZ}}+n$. For instance, a nondegenerate maximum of an autonomous Hamiltonian with small eigenvalues has degree $2 n$. Thus, we have a grading preserving isomorphism between the total Floer homology $\operatorname{HF}_{*}(H)$ and the quantum homology $\mathrm{HQ}_{*}(M)$.

Fix a ground field $\mathbb{F}$, eg, $\mathbb{Z}_{2}$ or $\mathbb{C}$ or $\mathbb{Q}$. Throughout the paper, $H_{*}(M)$ denotes the homology $H_{*}(M ; \mathbb{F})$ with coefficients in $\mathbb{F}$ and the Floer and quantum homology groups are also taken over the field $\mathbb{F}$.

2.2.1 Floer homology Let $H$ be a nondegenerate Hamiltonian on $M$. Denote by $\mathrm{CF}_{k}^{(-\infty, b)}(H)$, where $b \in(-\infty, \infty]$ is not in $\mathcal{S}(H)$, the vector space of formal sums

$$
\sum_{\bar{x} \in \overline{\mathcal{P}}(H)} \alpha_{\bar{x}} \bar{x} .
$$

Here $\alpha_{\bar{x}} \in \mathbb{F}$ and $\mu_{\mathrm{CZ}}(\bar{x})=k-n$ and $\mathcal{A}(\bar{x})<b$. Furthermore, we require, for every $a \in \mathbb{R}$, the number of terms in this sum with $\alpha_{\bar{x}} \neq 0$ and $\mathcal{A}(\bar{x})>a$ to be finite. The graded $\mathbb{F}$-vector space $\mathrm{CF}_{*}^{(-\infty, b)}(H)$ is endowed with the Floer differential counting the antigradient trajectories of the action functional in the standard way once a (timedependent) almost complex structure compatible with $\omega$ is fixed and the regularity 
requirements are satisfied; see, eg, Hofer and Salamon [32], McDuff and Salamon [42] and Salamon [49]. Thus, we obtain a filtration of the total Floer complex $\mathrm{CF}_{*}(H):=$ $\mathrm{CF}_{*}^{(-\infty, \infty)}(H)$. Furthermore, we set $\mathrm{CF}_{*}^{(a, b)}(H):=\mathrm{CF}_{*}^{(-\infty, b)}(H) / \mathrm{CF}_{*}^{(-\infty, a)}(H)$, where $-\infty \leq a<b \leq \infty$ are not in $\mathcal{S}(H)$. The resulting homology, the filtered Floer homology of $H$, is denoted by $\mathrm{HF}_{*}^{(a, b)}(H)$ and by $\mathrm{HF}_{*}(H)$ when $(a, b)=(-\infty, \infty)$. This construction extends to all, not necessarily nondegenerate, Hamiltonians by continuity. Here, for the sake of simplicity, we assume that $M$ is rational. Let $H$ be an arbitrary (one-periodic in time) Hamiltonian on $M$ and let the end points $a$ and $b$ of the action interval be outside $\mathcal{S}(H)$. By definition, we set

$$
\mathrm{HF}_{*}^{(a, b)}(H)=\mathrm{HF}_{*}^{(a, b)}(\tilde{H}),
$$

where $\widetilde{H}$ is a nondegenerate, small perturbation of $H$. It is well known that the right hand side in (2-3) is independent of $\widetilde{H}$ as long as the latter is sufficiently close to $H$. (It is essential that $a$ and $b$ are not in $\mathcal{S}(H)$ and $M$ is rational.) Working with filtered Floer homology, we will always assume that the end points of the action interval are not in the action spectrum and, if $H$ is not nondegenerate, the ambient manifold $M$ is rational.

When $a<b<c$, we have $\mathrm{CF}_{*}^{(b, c)}(H)=\mathrm{CF}_{*}^{(a, c)}(H) / \mathrm{CF}_{*}^{(a, b)}(H)$, and thus obtain the long exact sequence

$$
\cdots \rightarrow \mathrm{HF}_{*}^{(a, b)}(H) \rightarrow \mathrm{HF}_{*}^{(a, c)}(H) \rightarrow \mathrm{HF}_{*}^{(b, c)}(H) \rightarrow \cdots .
$$

The total Floer complex and homology are modules over the Novikov ring $\Lambda$. In this paper, the latter is defined as follows. Let $\omega(A)$ and $\left\langle c_{1}(M), A\right\rangle$ denote the integrals of $\omega$ and, respectively, $c_{1}(M)$ over a cycle $A$. Set

$$
I_{\omega}(A)=-\omega(A) \quad \text { and } \quad I_{c_{1}}(A)=-2\left\langle c_{1}(M), A\right\rangle,
$$

where $A \in \pi_{2}(M)$ and

$$
\Gamma=\frac{\pi_{2}(M)}{\operatorname{ker} I_{\omega} \cap \operatorname{ker} I_{c_{1}}} .
$$

Thus, $\Gamma$ is the quotient of $\pi_{2}(M)$ by the equivalence relation where the two spheres $A$ and $A^{\prime}$ are considered to be equivalent if $\omega(A)=\omega\left(A^{\prime}\right)$ and $\left\langle c_{1}(M), A\right\rangle=$ $\left\langle c_{1}(M), A^{\prime}\right\rangle$. The homomorphisms $I_{\omega}$ and $I_{c_{1}}$ defined originally on $\pi_{2}(M)$ descend to $\Gamma$. (With this convention $I_{\omega}(A)$ and $I_{c_{1}}(A)$ have the same sign when $M$ is monotone and opposite signs when $M$ is negative monotone.) The group $\Gamma$ acts on $\mathrm{CF}_{*}(H)$ and on $\mathrm{HF}_{*}(H)$ via recapping: an element $A \in \Gamma$ acts on a capped oneperiodic orbit $\bar{x}$ of $H$ by attaching the sphere $A$ to the original capping. We denote the resulting capped orbit by $\bar{x} \#$. Then,

$$
\operatorname{deg}(\bar{x} \# A)=\operatorname{deg}(\bar{x})+I_{c_{1}}(A) \quad \text { and } \quad \mathcal{A}_{H}(\bar{x} \# A)=\mathcal{A}_{H}(\bar{x})+I_{\omega}(A) .
$$


The Novikov ring $\Lambda$ is a certain completion of the group ring of $\Gamma$. Namely, $\Lambda$ is comprised of formal linear combinations $\sum \alpha_{A} e^{A}$, where $\alpha_{A} \in \mathbb{F}$ and $A \in \Gamma$, such that for every $a \in \mathbb{R}$ the sum contains only finitely many terms with $I_{\omega}(A)>a$ and, of course, $\alpha_{A} \neq 0$. (The appearance of $e^{A}$ rather than just $A$ is due to the fact that we use addition to denote the product in $\Gamma$ and multiplication to denote the product in $\Lambda$.) The Novikov ring $\Lambda$ is graded by setting $\operatorname{deg}\left(e^{A}\right)=I_{c_{1}}(A)$ for $A \in \Gamma$. The action of $\Gamma$ turns $\mathrm{CF}_{*}(H)$ and $\mathrm{HF}_{*}(H)$ into $\Lambda$-modules. Note that in general this action is defined only on the total Floer complex and homology and does not extend to their filtered counterparts.

Example 2.1 Assume that $M$ is monotone or negative monotone or rational with $N=\infty\left(\right.$ ie, $\left.\left.c_{1}(M)\right|_{\pi_{2}(M)}=0\right)$ and $\lambda_{0}<\infty\left(\right.$ ie, $\left.\left.\omega\right|_{\pi_{2}(M)} \neq 0\right)$. Then $\Gamma \cong \mathbb{Z}$ with the generator $A$ such that $I_{\omega}(A)=\lambda_{0}>0$ (ie, $\left.\omega(A)=-\lambda_{0}\right)$ and $I_{c_{1}}(A)= \pm 2 N$ when $N<\infty$. (The positive sign for monotone manifolds.) When $N=\infty$, we have $I_{c_{1}}(A)=0$. Furthermore, $\Lambda$ is the ring of Laurent series $\mathbb{F}[q] \llbracket q^{-1} \rrbracket$ with $q=e^{A}$, where $\operatorname{deg}(q)= \pm 2 N$ and $I_{\omega}(q)=\lambda_{0}$. When $M$ is symplectically aspherical, $\Gamma=0$ and $\Lambda \cong \mathbb{F}$.

Concluding this discussion of Floer homology, two remarks are due.

Remark 2.2 The complex $\mathrm{CF}_{*}^{(a, b)}(H)$ can be thought of as the vector space formed by the formal sums (2-2), where, in addition to other requirements, $a<\mathcal{A}(\bar{x})<b$. (Such a sum is necessarily finite if $a>-\infty$.) This point of view, taken in, eg, [22; 26; 29], enables one to relax the compactness requirement on $M$ in the construction of the filtered Floer homology. Namely, let $M$ be weakly monotone, rational, and geometrically bounded (see, eg, Audin and Lafontaine [2] and Cieliebak, Ginzburg and Kerman [6]). Assume furthermore that $H$ is compactly supported and $(a, b)$ contains no point of the subgroup $\left\langle\omega, \pi_{2}(M)\right\rangle$. (The latter condition forces $M$ to be rational and reduces to $0 \notin(a, b)$ when $M$ is symplectically aspherical.) Then the above construction of the Floer homology goes through word-for-word with the new definition of $\mathrm{CF}_{*}^{(a, b)}(H)$ once we notice that in (2-3) it suffices to only require the capped periodic orbits of $\widetilde{H}$ with action in $(a, b)$ to be nondegenerate and that this requirement holds for a generic perturbation $\widetilde{H}$. This observation is used in the proof of Theorem 1.17 in Section 5.

Remark 2.3 The condition that $M$ is rational in the definition of filtered Floer homology of general Hamiltonians is purely technical and can easily be eliminated although (2-3) can no longer be used. The reason is that once the rationality requirement is dropped, the right hand side of (2-3) depends in general on the choice of $\widetilde{H}$ no 
matter how close the latter is to $H$. Hence, instead of using (2-3), one can define $\mathrm{HF}_{*}^{(a, b)}(H)$ as the limit of $\mathrm{HF}_{*}^{(a, b)}(\tilde{H})$ over a certain class of perturbations $\tilde{H}$; cf [ 54 ; 55]. For instance, we may require that $\widetilde{H} \leq H$ and $a$ and $b$ are outside $\mathcal{S}(\widetilde{H})$. Then the condition that $a$ and $b$ are not in $\mathcal{S}(H)$ is irrelevant and $\operatorname{HF}_{*}^{(a, b)}(H)$ becomes very sensitive to variations of $a$ and $b$ and of $H$; cf Section 2.2.2. Throughout the paper, we mainly restrict our attention to the rational case, unless $H$ is assumed to be nondegenerate, for the rationality requirement enters the proofs in other, more essential, ways.

2.2.2 Homotopy maps A homotopy of Hamiltonians on $M$ is a family of (periodic in time) Hamiltonians $H^{s}$ smoothly parametrized by $s \in \mathbb{R}$ and a family of almost complex structures $J^{s}$, compatible with $\omega$, such that $H^{s}$ and $J^{s}$ are independent of $s$ for large positive and negative values of $s$. Throughout this paper, we suppress the family $J^{S}$ in the notation and think of a homotopy of Hamiltonians on $M$ as a family of (periodic in time) Hamiltonians $H^{S}$ smoothly parametrized by [0, 1]. (Such a family can be easily turned into one smoothly parametrized by $\mathbb{R}$.) It is well known that, when $H_{0}$ and $H_{1}$ are nondegenerate and certain (generic) regularity requirements are met, a homotopy gives rise to a chain map of Floer complexes

$$
\Psi: \mathrm{CF}_{*}\left(H^{0}\right) \rightarrow \mathrm{CF}_{*}\left(H^{1}\right)
$$

that induces an isomorphism on the level of total Floer homology; see, eg, McDuff and Salamon [42] and references therein. The map $\Psi$ does not preserve the action filtration. However, a standard estimate shows that $\Psi$ increases the action by no more than

$$
E:=\int_{-\infty}^{\infty} \int_{S^{1}} \max _{M} \partial_{s} H_{t}^{s} d t d s
$$

As a consequence, for every $c \geq E$, the homotopy induces a map, which we will also denote by $\Psi$ or $\Psi_{H^{0}, H^{1}}$, of the filtered Floer homology, shifting the action filtration by $c$ :

$$
\Psi_{H^{0}, H^{1}}: \mathrm{HF}_{*}^{(a, b)}\left(H^{0}\right) \rightarrow \mathrm{HF}_{*}^{(a+c, b+c)}\left(H^{1}\right) .
$$

This map need not be an isomorphism. For instance, $\Psi=0$ if $c>b-a$.

Example 2.4 Two particular cases of this construction are of interest for us. The first case is that of a monotone decreasing homotopy, ie, a homotopy with $\partial_{s} H_{t}^{s} \leq 0$. In this case, we can take $c=0$, and thus we obtain a map preserving the action filtration. The second case is that of a linear homotopy $H^{s}=s H^{1}+(1-s) H^{0}$. Here we can take $c=E=\int \max \left(H^{1}-H^{0}\right) d t$. In what follows, whenever a homotopy from $H^{0}$ to $H^{1} \geq H^{0}$ is considered, this is always a monotone linear homotopy, unless specified otherwise. 
A (nonmonotone) homotopy $H^{s}$ from $H^{0}$ to $H^{1}$ with $a$ and $b$ outside $\mathcal{S}\left(H^{s}\right)$ for all $s$ gives rise to an isomorphism between the groups $\mathrm{HF}_{*}^{(a, b)}\left(H^{s}\right)$, and hence, in particular,

$$
\mathrm{HF}_{*}^{(a, b)}\left(H^{0}\right) \cong \mathrm{HF}_{*}^{(a, b)}\left(H^{1}\right) ;
$$

see Viterbo [57] and also Biran, Polterovich and Salamon [4] and Ginzburg [22]. This isomorphism is not induced by the homotopy in the same sense as $\Psi_{H^{0}, H^{1}}$, but is constructed by breaking the homotopy $H^{s}$ into a composition of nearly constant homotopies for which (2-4) is essentially immediate. The isomorphism (2-4) is welldefined, ie, it is completely determined by the homotopy and independent of the choices made in its construction. Furthermore, the isomorphism commutes with the maps from the long exact sequence, provided that all three points $a<b<c$ are outside $\mathcal{S}\left(H^{s}\right)$ for all $s$. When $H^{s}$ is a decreasing homotopy, the isomorphism (2-4) coincides with $\Psi_{H^{0}, H^{1}}$.

Example 2.5 A homotopy $H^{s}$ is said to be isospectral if $\mathcal{S}\left(H^{s}\right)$ is independent of $s$. In this case, the isomorphism (2-4) is defined for any $a<b$ outside $\mathcal{S}\left(H^{s}\right)$.

For instance, let $\eta_{s}^{t}$, where $t \in S^{1}$ and $s \in[0,1]$, be a family of loops of Hamiltonian diffeomorphisms based at id, ie, $\eta_{s}^{0}=\mathrm{id}$ for all $s$. In other words, $\eta_{s}^{t}$ is a based homotopy from the loop $\eta_{0}^{t}$ to the loop $\eta_{1}^{t}$. Let $G_{t}^{s}$ be a family of one-periodic Hamiltonians generating these loops and let $H$ be a fixed one-periodic Hamiltonian. Then $H^{s}:=G^{s} \# H$ is an isospectral homotopy, provided that $G^{s}$ are suitably normalized. (Namely, $\mathcal{A}\left(G^{s}\right)=0$ for all $s$; see Section 2.3.)

When $K \geq H^{s}$ for all $s$, the isomorphism (2-4) intertwines monotone homotopy homomorphisms from $K$ to $H^{0}$ and to $H^{1}$, ie, the diagram

$$
\begin{aligned}
& \mathrm{HF}_{*}^{(a, b)}(K)_{\Psi_{K, H^{0}}}
\end{aligned}
$$

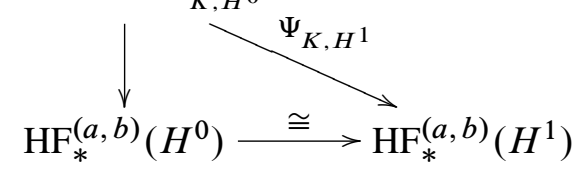

is commutative. (See, eg, Ginzburg [20] for more details.) The assumption that $K \geq H^{s}$ for all $s$ is essential here: the maps need not commute if we only require that $K \geq H^{0}$ and $K \geq H^{1}$.

2.2.3 Quantum homology The total Floer homology $\operatorname{HF}_{*}(H)$, equipped with the pair-of-pants product, is an algebra over the Novikov ring $\Lambda$. This algebra is isomorphic 
to the (small) quantum homology $\mathrm{HQ}_{*}(M)$; see, eg, McDuff and Salamon [42] and Piunikhin, Salamon and Schwarz [47]. On the level of $\Lambda$-modules, we have

$$
\mathrm{HQ}_{*}(M)=H_{*}(M) \otimes_{\mathbb{F}} \Lambda
$$

with the tensor product grading. Thus, $\operatorname{deg}\left(x \otimes e^{A}\right)=\operatorname{deg}(x)+I_{c_{1}}(A)$, where $x \in$ $H_{*}(M)$ and $A \in \Gamma$. The isomorphism between $\operatorname{HF}_{*}(H)$ and $\mathrm{HQ}_{*}(M)$ is defined via the PSS-homomorphism [47; 42]. Alternatively, it can be obtained from a homotopy of $H$ to an autonomous $C^{2}$-small Hamiltonian (under slightly more restrictive conditions than weak monotonicity [32]) or with a somewhat different definition of the total Floer homology (as the limit of $\operatorname{HF}_{*}^{(a, b)}(H)$ as $a \rightarrow-\infty$ and $b \rightarrow \infty$ [45]).

The quantum product $x * y$ of two elements $H_{*}(M)$ is defined as

$$
x * y=\sum_{A \in \Gamma}(x * y)_{A} e^{A},
$$

where the class $(x * y)_{A} \in H_{*}(M)$ is determined by the condition that

$$
(x * y)_{A} \circ z=\mathrm{GW}_{A, 3}^{M}(x, y, z)
$$

for all $z \in H_{*}(M)$. Here $\circ$ stands for the intersection index and $\mathrm{GW}_{A, 3}^{M}$ is the corresponding Gromov-Witten invariant [42]. Somewhat informally, the class $(x * y)_{A}$ can be described as follows. Let $J$ be a generic almost complex structure compatible with $\omega$. Fix generic cycles $X$ and $Y$ in $M$ representing the classes $x$ and $y$, respectively. For the sake of simplicity, we assume that $X$ and $Y$ are embedded. Let $\mathcal{M}$ be (the compactification of) the moduli space of $J$-holomorphic curves $u: \mathbb{C P} \mathbb{P}^{1} \rightarrow M$ in the class $A$ such that $u(0) \in X$ and $u(1) \in Y$. Then the homology class $(x * y)_{A}$ is represented by the cycle (or rather a pseudo-cycle) $\mathrm{ev}_{\infty}: \mathcal{M} \rightarrow M$ sending $u$ to $u(\infty)$; see, eg, McDuff and Salamon [42] for more details.

Note that $(x * y)_{0}=x \cap y$, where $\cap$ stands for the intersection product and $x$ and $y$ are ordinary homology classes. Furthermore,

$$
\begin{aligned}
\operatorname{deg}(x * y) & =\operatorname{deg}(x)+\operatorname{deg}(y)-2 n, \\
\operatorname{deg}(x * y)_{A} & =\operatorname{deg}(x)+\operatorname{deg}(y)-2 n-I_{c_{1}}(A) .
\end{aligned}
$$

Also observe that $I_{\omega}(A)=-\omega(A)<0$ whenever $A \neq 0$ can be represented by a holomorphic curve. Thus, in (2-7), it suffices to limit the summation to the negative cone $I_{\omega}(A) \leq 0$. In particular, in the setting of Example 2.1, we can write

$$
x * y=x \cap y+\sum_{k>0}(x * y)_{k} q^{-k} .
$$


Here, $\operatorname{deg}(x * y)_{k}=\operatorname{deg}(x)+\operatorname{deg}(y)-2 n \pm 2 N k$ when $N<\infty$, with the positive or negative sign depending on whether $M$ is positive or negative monotone. This sum is finite. When $N=\infty$ (ie, $\left.c_{1}(M)\right|_{\pi_{2}(M)}=0$ ), we have $\operatorname{deg}(x * y)_{k}=\operatorname{deg}(x)+$ $\operatorname{deg}(y)-2 n$.

The product $*$ extends to a $\Lambda$-linear, associative, graded-commutative product on $\mathrm{HQ}_{*}(M)$. The fundamental class $[M]$ is the unit in the algebra $\mathrm{HQ}_{*}(M)$. Thus, $q x=$ $(q[M]) * x$, where $q \in \Lambda$ and $x \in H_{*}(M)$, and $\operatorname{deg}(q x)=\operatorname{deg}(q)+\operatorname{deg}(x)$. By the very definition of $\mathrm{HQ}_{*}(M)$, the ordinary homology $H_{*}(M)$ is canonically embedded in $\mathrm{HQ}_{*}(M)$. The group of symplectomorphisms acts on the algebra $\mathrm{HQ}_{*}(M)$ via its action on $H_{*}(M)$ and, clearly, symplectomorphisms isotopic to id (in this group) act trivially.

Example 2.6 Assume that $N>2 n$. Then, as immediately follows from (2-8), the quantum product coincides with the intersection product: $x * y=x \cap y$. There are numerous examples of closed, negative monotone manifolds with $N>2 n$. (Among such manifolds is, for instance, the hypersurface $z_{0}^{k}+\cdots+z_{n}^{k}=0$ in $\mathbb{C} \mathbb{P}^{n}$ with $N=k-(n+1)>2 n$.) However, to the best of the authors knowledge, no closed, monotone manifolds with $N>n+1$ are known. In a similar vein, as is easy to see, $x * y=x \cap y$ when $M$ is negative monotone and $N \geq n$; cf Lê and Ono [37].

Example 2.7 Let $M=\mathbb{C P}^{n}$. Then $N=n+1$ and, in the notation of Example 2.1, $\mathrm{HQ}_{*}\left(\mathbb{C} \mathbb{P}^{n}\right)$ is the quotient of $\mathbb{F}[u] \otimes \Lambda$, where $u$ is the generator of $\left.H_{2 n-2}(\mathbb{C P})^{n}\right)$, by the ideal generated by the relation $u^{n+1}=q^{-1}[M]$ [42]. Thus, $u^{k}=u \cap \cdots \cap u$ ( $k$ times) when $0 \leq k \leq n$ and $[\mathrm{pt}] * u=q^{-1}[M]$. For further examples of calculations of quantum homology and relevant references we refer the reader to, eg, McDuff and Salamon [42].

Finally, let us extend the map $I_{\omega}$ from $\Gamma$ to $\mathrm{HQ}_{*}(M)$ and $\Lambda$ by setting

$$
I_{\omega}(x)=\max \left\{I_{\omega}(A) \mid x_{A} \neq 0\right\}
$$

for $x=\sum x_{A} e^{A} \in \mathrm{HQ}_{*}(M)$ and $I_{\omega}(0)=-\infty$. The extension to $\Lambda$ is defined by a similar formula or can be obtained by restricting $I_{\omega}$ from $\mathrm{HQ}_{*}(M)$ to $\Lambda \cong[M] \Lambda$. The map $I_{\omega}$ is a valuation:

$$
I_{\omega}(x+y) \leq \max \left\{I_{\omega}(x), I_{\omega}(y)\right\}
$$

and $I_{\omega}(\alpha x)=I_{\omega}(x)$ if $\alpha \in \mathbb{F}$ is nonzero.

Remark 2.8 Note in conclusion that the definition of the Novikov ring $\Lambda$ adapted in this paper is by no means standard in the context of quantum homology, although it is 
among the most natural choices as far as Floer homology is concerned. McDuff and Salamon [42] offer a detailed discussion of a variety of choices of the Novikov ring.

\subsection{Contractible loops of Hamiltonian diffeomorphisms}

Our goal in this section is to recall a few, mainly well-known, facts about contractible loops of Hamiltonian diffeomorphisms and, more specifically, about the action of such loops on the Floer complex of a Hamiltonian.

In what follows, a loop $\eta^{t}, t \in S^{1}$, is always assumed to be based at the identity and equipped with a (homotopy type of) contraction $\eta_{s}$ of $\eta_{1}=\eta$ to $\eta_{0} \equiv \mathrm{id}$, ie, viewed as an element of the universal covering of the identity component in the space of loops based at id. Furthermore, when the action of a Hamiltonian on closed curves is essential, a loop $\eta$ will also be equipped with a loop of Hamiltonians $G_{t}^{s}$ generating $\eta_{s}^{t}$. Since $G_{t}^{0}$ generates the identity Hamiltonian diffeomorphism, we may assume without loss of generality that $G_{t}^{0} \equiv 0$.

\subsubsection{Action of loops on capped closed curves and on Floer homology With the} above conventions in mind, we observe that every orbit $y=\eta^{t}(p)$ of a loop $\eta$ automatically comes with a capping arising from the contraction of $\eta$ to id. Trivializing $T M$ along such a capping, we have the Maslov index of the loop $\eta$ (ie, the Maslov index of the loop of linear symplectic transformations $\left.d \eta^{t}\right|_{\eta^{t}(p)}: T_{p} M \rightarrow T_{p} M$ ) well-defined. This index is obviously equal to zero. Furthermore, once the loop of Hamiltonians $G_{t}^{s}$ generating $\eta_{s}^{t}$ is fixed, the action $\mathcal{A}(G):=\mathcal{A}_{G}(\bar{y})$ of $G$ on the capped orbit $\bar{y}$ is independent of the initial point $p$. (This action depends on the choice of the Hamiltonians $G_{t}^{s}$. More specifically, $\mathcal{A}(G)$ is determined by the Hamiltonians $G_{t}=G_{t}^{1}$ and by $\eta$ regarded as an element of the universal covering.)

Let $\bar{x}$ be an arbitrary capped closed curve in $M$. We can view the capping of $x$ as a map $[0,1] \times S^{1} \rightarrow M$ sending $[0,1] \times\{0\}$ and $\{0\} \times S^{1}$ to $p=x(0)$ and equal to $x$ on $\{1\} \times S^{1}$, ie, a family of closed curves $x_{s}, s \in[0,1]$, connecting $x_{0} \equiv p$ with $x_{1}=x$ and such that $x_{s}(0)=p$ for all $s$. Then $\left.t \mapsto \eta^{t}(x(t))\right)$ is again a closed curve with capping $(t, s) \mapsto \eta_{s}^{t}\left(x_{s}(t)\right)$, which we denote by $\Phi_{\eta}(\bar{x})$ or $\Phi_{G}(\bar{x})$. We say that the loop $\eta$ sends the capped curve $\bar{x}$ to $\Phi_{\eta}(\bar{x})$.

To apply this observation to Floer homology, consider a one-periodic Hamiltonian $H_{t}$. The time-dependent flow $\eta^{t} \circ \varphi_{H}^{t}$ is generated by the Hamiltonian $G \# H$ and its timeone map coincides with $\varphi_{H}$ in the universal covering of the group of Hamiltonian diffeomorphisms. Hence, the map $\Phi_{G}$ gives rise to a one-to-one correspondence between capped one-periodic orbits of $H$ and those of $G \# H$. Moreover, this map induces an isomorphism of Floer complexes. More precisely, let $J_{t}$ be a time-dependent 
almost complex structure on $M$. Then $\Phi_{G}$ induces an isomorphism between the Floer complex of $(H, J)$ and the Floer complex of $G \# H$ with the almost complex structure $\widetilde{J}_{t}:=d \eta^{t} \circ J_{t} \circ\left(d \eta^{t}\right)^{-1}$. This isomorphism preserves the grading (since the Maslov index of $\eta$ is zero) and shifts the action filtration by $\mathcal{A}(G)$. Hence, in particular, we obtain an isomorphism

$$
\mathrm{HF}_{*}^{(a, b)}(H) \cong \mathrm{HF}_{*}^{(a+\mathcal{A}(G), b+\mathcal{A}(G))}(G \# H) .
$$

On the level of total Floer homology, where the action filtration is essentially ignored, this isomorphism coincides with the homotopy isomorphism $\Psi_{H, G \# H}$. (This is equivalent to the fact that the Seidel representation is well-defined, ie, trivial for contractible loops [42].)

2.3.2 Extension of loops The next two geometrical results discussed in this section concern the existence and extension of loops with specific properties and are used in the proof of Theorem 1.17. Here, we mainly follow [20], taking into account cappings of periodic orbits.

The first of these results asserts that every capped closed curve is an orbit of a contractible loop of Hamiltonian diffeomorphisms. More precisely, we have:

Proposition 2.9 Let $\bar{x}$ be a capped closed curve in $M$. Then there exists a contractible loop of Hamiltonian diffeomorphisms $\eta$ fixing $p=x(0)$ such that $\Phi_{\eta}(\bar{x})$ is a constant curve $p$ with trivial capping.

Proof As above, we can view the capping of $x$ as a smooth family of closed curves $x_{s}: S^{1} \rightarrow M, s \in[0,1]$, connecting the constant loop $x_{0} \equiv p$ to $x_{1}=x$. It is easy to show that there exists a smooth family of Hamiltonians $G_{t}^{s}$ such that for every $t$, the curve $s \mapsto x_{s}(t)$ is an integral curve of $G_{t}^{s}$ with respect to $s$, ie, $x_{s}=\varphi_{G}^{s}(p)$. Let $\eta^{t}=\varphi_{G}^{1}$ be the time-one flow of this family, parametrized by $t \in S^{1}$. Then $x=\eta^{t}(p)$. The family of Hamiltonians $G_{t}^{s}$ can be chosen so that $G_{s, 0} \equiv 0 \equiv G_{s, 1}$. Then $\eta^{t}$ is a loop of Hamiltonian diffeomorphisms with $\eta^{0}=\mathrm{id}=\eta^{1}$. As readily follows from the construction, the loop $\eta$ is contractible.

The second result gives a necessary and sufficient condition for the existence of an extension of a loop of local Hamiltonian diffeomorphisms to a global loop.

Consider a loop $\eta^{t}$ of (the germs of) Hamiltonian diffeomorphisms at $p \in M$ generated by $G$. In other words, the maps $\eta^{t}$ and the Hamiltonian $G$ are defined on a small neighborhood of $p$ and $\eta^{t}(p)=p$ for all $t \in S^{1}$. Then the action $\mathcal{A}(G)$ and the Maslov index $\mu(\eta)$ are introduced exactly as above with the orbit $\bar{x}$ taken sufficiently close 
to $p$. (In fact, we can set $\bar{x} \equiv p$ with trivial capping. Hence, $\mathcal{A}(G)=\int_{0}^{1} G_{t}(p) d t$ and $\mu(\eta)$ is just the Maslov index of the loop $d \eta_{p}^{t}$ in $\operatorname{Sp}\left(T_{p} W\right)$.) Note that in this case $\mu(\eta)$ need not be zero.

Proposition 2.10 Let $\eta^{t}, t \in S^{1}$, be a loop of germs of Hamiltonian diffeomorphisms at $p \in M$. The following conditions are equivalent:

(i) The loop $\eta$ extends to a loop of global Hamiltonian diffeomorphisms of $M$, contractible in the class of loops fixing $p$.

(ii) The loop $\eta$ is contractible in the group of germs of Hamiltonian diffeomorphisms at $p$.

(iii) $\mu(\eta)=0$.

Proof The implications (i) $\Rightarrow$ (ii) $\Rightarrow$ (iii) are obvious. To prove that (iii) $\Rightarrow$ (ii), we identify a neighborhood of $p$ in $M$ with a neighborhood of the origin in $\mathbb{R}^{2 n}$. Then, as is easy to see, the loop $\eta^{t}$ is homotopy equivalent to its linearization $d \eta_{p}^{t}$, a loop of (germs of) linear maps. By the definition of the Maslov index, $d \eta^{t}$ is contractible in $\operatorname{Sp}\left(\mathbb{R}^{2 n}\right)$ if and only if $\mu(\eta):=\mu\left(d \eta_{p}\right)=0$.

To complete the proof of the proposition, it remains to show that (ii) $\Rightarrow$ (i). To this end, let us first analyze the case where $\eta^{t}$ is $C^{1}$-close to the identity (and hence contractible). Fixing a small neighborhood $U$ of $p$, we identify a neighborhood of the diagonal in $U \times U$ with a neighborhood of the zero section in $T^{*} U$. Then the graphs of $\eta^{t}$ in $U \times U$ turn into Lagrangian sections of $T^{*} U$. These sections are the graphs of exact forms $d f_{t}$ on $U$, where all $f_{t}$ are $C^{2}$-small and $f_{0} \equiv 0 \equiv f_{1}$. Then we extend (the germs of) the functions $f_{t}$ to $C^{2}$-small functions $\tilde{f}_{t}$ on $M$ such that $\widetilde{f}_{0} \equiv 0 \equiv \widetilde{f}_{1}$. The graphs of $d \widetilde{f}_{t}$ in $T^{*} M$ form a loop of exact Lagrangian submanifolds which are $C^{1}$-close to the zero section. Thus, this loop can be viewed as a loop of Hamiltonian diffeomorphisms of $M$. It is clear that the resulting loop is contractible in the class of loops fixing $p$.

To deal with the general case, consider a contraction of $\eta$ to id, ie, a family $\eta_{s}$, $s \in[0,1]$, of local loops with $\eta_{0} \equiv$ id and $\eta_{1}^{t}=\eta^{t}$. Let $0=s_{0}<s_{1}<\cdots<s_{k}=1$ be a partition of the interval $[0,1]$ such that the loops $\eta_{s_{i}}$ and $\eta_{s_{i+1}}$ are $C^{1}$-close for all $i=0, \ldots, k-1$. In particular, the loop $\eta_{s_{1}}$ is $C^{1}$-close to $\eta_{0}=\mathrm{id}$, and thus extends to a contractible loop $\tilde{\eta}_{s_{1}}$ on $M$. Arguing inductively, assume that a contractible extension $\tilde{\eta}_{s_{i}}$ of $\eta_{s_{i}}$ has been constructed. Consider the loop $\eta^{t}=\eta_{s_{i+1}}^{t}\left(\eta_{s_{i}}^{t}\right)^{-1}$ defined near $p$. This loop is $C^{1}$-close to the identity, for $\eta_{s_{i+1}}$ and $\eta_{s_{i}}$ are $C^{1}$-close. Hence, $\eta$ extends to a contractible loop $\tilde{\eta}$ on $M$. Then $\widetilde{\eta}_{s_{i+1}}^{t}:=\widetilde{\eta}^{t} \widetilde{\eta}_{s_{i}}^{t}$ is the required extension of $\eta_{s_{i+1}}$, contractible in the class of loops fixing $p$. 
Remark 2.11 In the proof of Theorem 1.17, we will also need the following variant of Proposition 2.10. Assume that $\eta$ is the germ of a loop near $p$ and the linearization of $\eta$ at $p$ is equal to the identity, ie, $d \eta_{p}^{t}=I$ for all $t$. (Hence, $\mu(\eta)=0$.) Then $\eta$ extends to a loop $\tilde{\eta}$ of global Hamiltonian diffeomorphisms of $M$ such that $\tilde{\eta}$ is contractible in the class of loops fixing $p$ and having identity linearization at $p$. This fact can be verified similarly to the proof of the implication (ii) $\Rightarrow$ (i).

\subsection{The mean index}

Let $\bar{x}$ be a capped one-periodic orbit of a Hamiltonian $H$ on $M$. (It suffices to have $H$ defined only on a neighborhood of $x$.) The mean index $\Delta_{H}(\bar{x}) \in \mathbb{R}$ measures the sum of rotations of the eigenvalues of $d\left(\varphi_{H}^{t}\right)_{x(t)}$ lying on the unit circle. Here $d\left(\varphi_{H}^{t}\right)_{x(t)}$ is interpreted as a path in the group of linear symplectomorphisms by using the trivialization of $T M$ along $x$, associated with the capping. (Similarly to our notation for the action functional, we write $\Delta_{H^{(k)}}(\bar{x})$ when $\bar{x}$ is $k$-periodic.) Referring the reader to [50] for a precise definition of $\Delta_{H}(\bar{x})$ and the proofs of its properties, we just recall here the following facts that are used in this paper.

(MI1) The iteration formula: $\Delta_{H^{(k)}}\left(\bar{x}^{k}\right)=k \Delta_{H}(\bar{x})$.

(MI2) Continuity: Let $\widetilde{H}$ be a $C^{2}$-small perturbation of $H$ and let $\bar{y}$ be a capped one-periodic orbit of $\tilde{H}$ close to $\bar{x}$. Then $\left|\Delta_{H}(\bar{y})-\Delta_{\tilde{H}}(\bar{x})\right|$ is small.

(MI3) The mean index formula: Assume that $x$ is nondegenerate. Then, as $k \rightarrow \infty$ through admissible iterations (see Section 4.2), $\mu_{\mathrm{CZ}}\left(H^{(k)}, \bar{x}^{k}\right) / k \rightarrow \Delta_{H}(\bar{x})$.

(MI4) Relation to the Conley-Zehnder index: Let $\bar{x}$ split into nondegenerate orbits $\bar{x}_{1}, \ldots, \bar{x}_{m}$ under a $C^{2}$-small, nondegenerate perturbation $\widetilde{H}$ of $H$. Then $\left|\mu_{\mathrm{CZ}}\left(\tilde{H}, \bar{x}_{i}\right)-\Delta_{H}(\bar{x})\right| \leq n$ for all $i=1, \ldots, m$. Moreover, these inequalities are strict when $x$ is weakly nondegenerate; see Salamon and Zehnder [50, page 1357]. In particular, if $x$ is nondegenerate, $\left|\mu_{\mathrm{CZ}}(H, \bar{x})-\Delta_{H}(\bar{x})\right|<n$.

(MI5) Additivity: Let $\bar{x}_{1}$ and $\bar{x}_{2}$ be one-periodic orbits of Hamiltonians $H_{1}$ and $H_{2}$ on manifolds $M_{1}$ and, respectively, $M_{2}$. Then $\Delta_{H_{1}+H_{2}}\left(\left(\bar{x}_{1}, \bar{x}_{2}\right)\right)=$ $\Delta_{H_{1}}\left(\bar{x}_{1}\right)+\Delta_{H_{2}}\left(\bar{x}_{2}\right)$, where $H_{1}+H_{2}$ is the naturally defined Hamiltonian on $M_{1} \times M_{2}$.

(MI6) Action of global loops: Assume that $G$ generates a contractible loop of Hamiltonian diffeomorphisms of $M$. Then $\Delta_{G \# H}\left(\Phi_{G}(\bar{x})\right)=\Delta_{H}(\bar{x})$.

(MI7) Action of local loops: Assume that $\bar{x}$ is a constant one-periodic orbit $x(t) \equiv p$ equipped with trivial capping and that $G$ generates a loop of Hamiltonian diffeomorphisms fixing $p$ and defined on a neighborhood of $p$. Then $\Delta_{G \# H}\left(\Phi_{G}(\bar{x})\right)=\Delta_{H}(\bar{x})+2 \mu$, where $\mu$ is the Maslov index of the loop $d\left(\varphi_{G}^{t}\right)_{p}$. 
(MI8) Index of strongly degenerate orbits: Assume that $x$ is strongly degenerate. Then $\Delta_{H}(\bar{x}) \in 2 \mathbb{Z}$. Moreover, when $\bar{x} \equiv p$ is a constant orbit as in (MI7) and $H$ is defined on a neighborhood of $p$ and generates a loop of Hamiltonian diffeomorphisms, we have $\Delta_{H}(p)=2 \mu$, where $\mu$ is the Maslov index of the loop $\varphi_{H}^{t}$.

The mean index spectrum $\mathcal{I}(H)$ of $H$ is the set $\left\{\Delta_{H}(\bar{x}) \mid \bar{x} \in \overline{\mathcal{P}}(H)\right\}$. This set is closed, but, in contrast with the action spectrum, need not have zero measure or even be nowhere dense. The mean index spectrum modulo $2 N$ is denoted by $\mathcal{I}_{2 N}(H)$. Thus, $\mathcal{I}_{2 N}(H)=\left\{\Delta_{H}(x) \in S_{2 N}^{1} \mid x \in \mathcal{P}(H)\right\}$. Similarly to (2-1), we have

$$
\frac{\Delta_{H^{(k)}}(\bar{x})}{k} \equiv \frac{\Delta_{H^{(l)}}(\bar{y})}{l} \bmod \mathbb{Q}
$$

if the $k$-periodic orbit $\bar{x}$ is geometrically identical to the $l$-periodic orbit $\bar{y}$.

\subsection{Local Floer homology}

In this section, we briefly recall the definition and basic properties of local Floer homology following mainly [20; 24], although this notion goes back to the original work of Floer ( eg $[15 ; 14]$ ) and has been revisited a number of times since then; see, eg, Poźniak [48, Section 3.3.4].

Let $\bar{x}$ be a capped isolated one-periodic orbit of a Hamiltonian $H: S^{1} \times M \rightarrow \mathbb{R}$. Pick a sufficiently small tubular neighborhood $U$ of $\gamma$ and consider a nondegenerate $C^{2}$-small perturbation $\tilde{H}$ of $H$ supported in $U$. Every (antigradient) Floer trajectory $u$ connecting two one-periodic orbits of $\widetilde{H}$ lying in $U$ is also contained in $U$, provided that $\|\tilde{H}-H\|_{C^{2}}$ and $\operatorname{supp}(\tilde{H}-H)$ are small enough. Thus, by the compactness and gluing theorems, every broken antigradient trajectory connecting two such orbits also lies entirely in $U$. Similarly to the definition of local Morse homology, the vector space (over $\mathbb{F}$ ) generated by one-periodic orbits of $\tilde{H}$ in $U$ is a complex with (Floer) differential defined in the standard way. The continuation argument $[42 ; 50]$ shows that the homology of this complex is independent of the choice of $\widetilde{H}$ and of the almost complex structure. We refer to the resulting homology group $\operatorname{HF}_{*}(H, \bar{x})$ as the local Floer homology of $H$ at $\bar{x}$.

Example 2.12 Assume that $\bar{x}$ is nondegenerate and $\mu_{\mathrm{CZ}}(\bar{x})=k$. Then $\operatorname{HF}_{l}(H, \bar{x})=\mathbb{F}$ when $l=k$ and $\operatorname{HF}_{l}(H, \bar{x})=0$ otherwise.

In the rest of this section, we list the basic properties of local Floer homology that are essential for what follows. 
(LF1) Let $H^{s}, s \in[0,1]$, be a family of Hamiltonians such that $x$ is a uniformly isolated one-periodic orbit for all $H^{s}$, ie, $x$ is the only periodic orbit of $H_{S}$, for all $s$, in some open set independent of $s$. Then $\operatorname{HF}_{*}\left(H^{s}, \bar{x}\right)$ is constant throughout the family: $\operatorname{HF}_{*}\left(H^{0}, \bar{x}\right)=\operatorname{HF}_{*}\left(H^{1}, \bar{x}\right)$.

Local Floer homology spaces are building blocks for filtered Floer homology. Namely, essentially by definition, we have the following:

(LF2) Assume that all one-periodic orbits $x$ of $H$ are isolated and $\operatorname{HF}_{k}(H, \bar{x})=0$ for some $k$ and all $\bar{x}$. Then $\operatorname{HF}_{k}(H)=0$. Moreover, let $M$ be rational and closed and let $c \in \mathbb{R}$ be such that all capped one-periodic orbits $\bar{x}_{i}$ of $H$ with action $c$ are isolated. (As a consequence, there are only finitely many orbits with action close to $c$.) Then, if $\epsilon>0$ is small enough,

$$
\mathrm{HF}_{*}^{(c-\epsilon, c+\epsilon)}(H)=\bigoplus_{i} \operatorname{HF}_{*}\left(H, \bar{x}_{i}\right)
$$

Just as ordinary Floer homology, the local Floer homology is completely determined by the time-one map generated by $H$ viewed as an element of the universal covering of the group of Hamiltonian diffeomorphisms:

(LF3) Let $\varphi_{G}^{t}$ be a contractible loop of Hamiltonian diffeomorphisms of $M$. Then

$$
\operatorname{HF}_{*}\left(G \# H, \Phi_{G}(\bar{x})\right)=\operatorname{HF}_{*}(H, \bar{x})
$$

for every isolated one-periodic orbit $x$ of $H$.

Furthermore, the Künneth formula holds for local Floer homology:

(LF4) Let $\bar{x}_{1}$ and $\bar{x}_{2}$ be capped one-periodic orbits of Hamiltonians $H_{1}$ and $H_{2}$ on symplectic manifolds $M_{1}$ and $M_{2}$. Then $\mathrm{HF}_{*}\left(H_{1}+H_{2},\left(\bar{x}_{1}, \bar{x}_{2}\right)\right)=$ $\mathrm{HF}_{*}\left(H_{1}, \bar{x}_{1}\right) \otimes \mathrm{HF}_{*}\left(H_{2}, \bar{x}_{2}\right)$, where $H_{1}+H_{2}$ is the naturally defined Hamiltonian on $M_{1} \times M_{2}$.

By definition, the support of $\operatorname{HF}_{*}(H, \bar{x})$ is the collection of integers $k$ such that $\mathrm{HF}_{k}(H, \bar{x}) \neq 0$. Clearly, the group $\operatorname{HF}_{*}(H, \bar{x})$ is finitely generated and hence supported in a finite range of degrees. The next observation, providing more precise information on the support of $\mathrm{HF}_{*}(H, \bar{x})$, is an immediate consequence of (MI4).

(LF5) The group $\mathrm{HF}_{*}(H, \bar{x})$ is supported in the range $\left[\Delta_{H}(\bar{x}), \Delta_{H}(\gamma)+2 n\right]$. Moreover, when $x$ is weakly nondegenerate, the support is contained in the open interval $\left(\Delta_{H}(\bar{x}), \Delta_{H}(\bar{x})+2 n\right)$. 
As is easy to see from the definition of local Floer homology, $H$ need not be a function on the entire manifold $M$ - it is sufficient to consider Hamiltonians defined only on a neighborhood of $x$. For the sake of simplicity, we focus on the particular case where $x(t) \equiv p$ is a constant orbit equipped with trivial capping. Thus, $d H_{t}(p)=0$ for all $t \in S^{1}$. Then (LF1), (LF4) and (LF5) still hold, and (LF3) takes the following form:

(LF6) Let $\varphi_{G}^{t}$ be a loop of Hamiltonian diffeomorphisms defined on a neighborhood of $p$ and fixing $p$. Then

$$
\mathrm{HF}_{*}(G \# H, p)=\mathrm{HF}_{*+2 \mu}(H, p),
$$

where $\mu$ is the Maslov index of the loop $t \mapsto d\left(\varphi_{G}^{t}\right)_{p} \in \operatorname{Sp}\left(T_{p} M\right)$.

\section{The Conley conjecture type results}

Our goal in this section is to prove three Conley conjecture type results - Theorems $1.1,1.3$ and 1.18 - and to illustrate our point that the arguments, in fact, concern the action or index spectra of the Hamiltonian in question. The proofs rely on Theorem 1.17, which is established in Section 5.

\subsection{The normalized action and mean index spectra}

The most direct way to obtain information about the number of periodic orbits via the action or mean index spectrum $\mathcal{S}_{\lambda_{0}}(H)$ and $\mathcal{I}_{2 N}(H)$, discussed in Sections 2.1.2 and 2.4, is by simply observing that the number of (not necessarily simple) geometrically distinct $k$-periodic orbits of $H$ is bounded from below by $\left|\mathcal{S}_{\lambda_{0}}\left(H^{(k)}\right)\right|$ and $\left|\mathcal{I}_{2 N}\left(H^{(k)}\right)\right|$. (Here we allow the cardinality $|\cdot|$ to assume infinite value, but we do not distinguish between different kinds of infinity.) Clearly, $H$ has infinitely many geometrically distinct periodic orbits whenever

(3-1) $\quad \limsup \left|\mathcal{S}_{\lambda_{0}}\left(H^{(k)}\right)\right|=\infty \quad$ or $\quad \limsup \left|\mathcal{I}_{2 N}\left(H^{(k)}\right)\right|=\infty$ as $k \rightarrow \infty$.

The spectra of the iterations $H^{(l)}$ and $H^{(k l)}$ are related via natural maps

$$
\mathcal{S}_{\lambda_{0}}\left(H^{(l)}\right) \rightarrow k \mathcal{S}_{\lambda_{0}}\left(H^{(l)}\right) \subset \mathcal{S}_{\lambda_{0}}\left(H^{(k l)}\right) .
$$

When $\left|\mathcal{P}^{\infty}(H)\right|<\infty$, the inclusions $k \mathcal{S}_{\lambda_{0}}\left(H^{(l)}\right) \subset \mathcal{S}_{\lambda_{0}}\left(H^{(k l)}\right)$ "stabilize", that is, $\mathcal{S}_{\lambda_{0}}\left(H^{(k d)}\right)=k \mathcal{S}_{\lambda_{0}}\left(H^{(d)}\right)$ for all $k$, where $d$ is the least common multiple of simple periods of $H$. Thus, we obtain the following criterion: Assume that $H$ has finitely many periodic orbits. Then, for every $k_{0}>0$ there exists $l>0$, divisible by $k_{0}$, such that $k \mathcal{S}_{\lambda_{0}}\left(H^{(l)}\right)=\mathcal{S}_{\lambda_{0}}\left(H^{(k l)}\right)$ for every $k$. Clearly, the same considerations apply when the action spectrum is replaced by the mean index spectrum $\mathcal{I}_{2 N}\left(H^{(k)}\right)$. 
Alternatively, to account for the role of recapping and iterations, it is sometimes convenient to interchange taking the lim sup and cardinality in (3-1) and put together the spectra of all iterations $H^{(k)}$ as follows.

Let $a \in(0, \infty]$. Consider the set of infinite sequences $a_{l}, a_{2 l}=2 a_{l}, a_{3 l}=3 a_{l}, \ldots$ in $S_{a}^{1}=\mathbb{R} / a \mathbb{Z}$, for all integers $l>0$. Let us call two sequences $\left\{a_{i l}\right\}$ and $\left\{a_{i l^{\prime}}^{\prime}\right\}$ equivalent if they coincide on their common domain of indices or, equivalently, $a_{d}=a_{d}^{\prime}$, where $d$ is the least common multiple of $l$ and $l^{\prime}$. The set of such sequences, up to this equivalence relation, is in one-to-one correspondence with $\mathbb{R} / a \mathbb{Q}$ via the map sending $\left\{a_{i l}\right\}$ to $a_{i l} /(i l)$. Here, when $a=\infty$, the quotient $\mathbb{R} / a \mathbb{Q}$ is taken to be $\mathbb{R}$.

Let now $H$ be a one-periodic Hamiltonian on a rational manifold $M$. Then every capped $k$-periodic orbit $\bar{x}$ of $H$ gives rise to a sequence $a_{i k}=\mathcal{A}_{H^{(i k)}}\left(\bar{x}^{i}\right)$. The resulting element of $\mathbb{R} / \lambda_{0} \mathbb{Q}$, which we will call the normalized action of $H$ on $\bar{x}$, is independent of capping and, by (2-1), two geometrically identical periodic orbits give rise to the same element. The set $\mathcal{S}_{\lambda_{0}}^{\infty}(H) \subset \mathbb{R} / \lambda_{0} \mathbb{Q}$ of all normalized actions of $H$ will be called the normalized action spectrum of $H$. The normalized mean index spectrum of $H$, denoted by $\mathcal{I}^{\infty}(H) \subset \mathbb{R} / 2 N \mathbb{Q}=\mathbb{R} / \mathbb{Q}$, is defined in a similar fashion. Finally note that the assumption that $H$ is one-periodic is not essential and the definitions extend to $k$-periodic Hamiltonians in an obvious way. It is clear from (2-1) and (2-9) that

and

$$
\begin{aligned}
& \left|\mathcal{P}^{\infty}(H)\right| \geq \limsup \left|\mathcal{S}_{\lambda_{0}}\left(H^{(k)}\right)\right| \geq\left|\mathcal{S}_{\lambda_{0}}^{\infty}(H)\right| \\
& \left|\mathcal{P}^{\infty}(H)\right| \geq \limsup \left|\mathcal{I}_{2 N}\left(H^{(k)}\right)\right| \geq\left|\mathcal{I}^{\infty}(H)\right| .
\end{aligned}
$$

Although these lower bounds are admittedly indiscriminating tools for keeping track of periodic orbits, they do naturally arise in the proofs of Theorem 1.3 and Theorem 1.18. Note also the inclusions $\mathcal{S}_{\lambda_{0}}^{\infty}\left(H^{(k)}\right) \subset \mathcal{S}_{\lambda_{0}}^{\infty}(H)$ and $\mathcal{I}^{\infty}\left(H^{(k)}\right) \subset \mathcal{I}^{\infty}(H)$ which hold for any integer $k>0$.

\subsection{Proof of Theorem 1.18}

The first assertion (i) is a consequence of the following result:

Proposition 3.1 Let $M$ and $H$ be as in Theorem 1.18(i) and all periodic orbits of $H$ are isolated. Then for every $l>0$ divisible by $k_{0}$ there exists $k>0$ such that $k \mathcal{S}_{\lambda_{0}}\left(H^{(l)}\right) \neq \mathcal{S}_{\lambda_{0}}\left(H^{(k l)}\right)$, ie, the action spectrum of $H^{(k)}$ never stabilizes.

Proof of Proposition 3.1 Let us focus on the case where $\lambda_{0}<\infty$. (The case of $\lambda_{0}=$ $\infty$, ie, $\left.\omega\right|_{\pi_{2}(M)}=0$ is treated in a similar way. Moreover, then $\lim \sup \left|\mathcal{S}\left(H^{(k)}\right)\right|=\infty$.) 
Furthermore, let us normalize $H$ so that the action on the symplectically degenerate maximum is equal to zero.

Arguing by contradiction, assume that there exists $l>0$, divisible by $k_{0}$, such that $k \mathcal{S}_{\lambda_{0}}\left(H^{(l)}\right)=\mathcal{S}_{\lambda_{0}}\left(H^{(k l)}\right)$ for every $k$. The Hamiltonian $F=H^{(l)}$ still has a symplectically degenerate maximum (with action value equal to zero), for an iteration of a symplectically degenerate maximum is again a symplectically degenerate maximum [24]. Thus, Theorem 1.17 applies to $F$. Finally, for the sake of simplicity, let us assume that $F$ is one-periodic, for the period can always be altered via a reparametrization.

The action spectrum $\mathcal{S}_{\lambda_{0}}(F)$ is finite since the periodic orbits of $H$, and hence of $F$, are isolated. Let

$$
\mathcal{S}_{\lambda_{0}}(F)=\left\{a_{1}, \ldots, a_{r}\right\} \cup \lambda_{0}\left\{p_{1} / q_{1}, \ldots, p_{s} / q_{s}\right\},
$$

where the first $r$ points $a_{i}$ are irrational modulo $\lambda_{0}$, the last $s$ points $p_{j} / q_{j}$ are rational and we require $p_{j}$ and $q_{j}$ to be mutually prime. Let $d$ be the least common multiple of $q_{1}, \ldots, q_{s}$. Then, due to our assumptions,

$$
\mathcal{S}_{\lambda_{0}}\left(F^{(k)}\right)=k \mathcal{S}_{\lambda_{0}}(F) \subset\left\{k a_{1}, \ldots, k a_{r}\right\} \cup \frac{\lambda_{0}}{d} \mathbb{Z}
$$

modulo $\lambda_{0}$. By Theorem 1.17 , for every sufficiently small $\epsilon>0$, there exists $k_{\epsilon}>0$ such that for every $k>k_{\epsilon}$, at least one of the points from $\mathcal{S}_{\lambda_{0}}\left(F^{(k)}\right)$ is in the arc $(0, \epsilon) \subset S^{1}$. When $\epsilon<1 / d$, this is one of the points $k a_{i}$. Denote by $p_{i}(m, \epsilon)$ the probability that the point $k a_{i}$ with $k_{\epsilon}<k \leq m$ is in the arc $(0, \epsilon)$. In other words, $p_{i}(m, \epsilon)$ is the number of integers $k \in\left(k_{\epsilon}, m\right]$, divided by $m-k_{\epsilon}$, such that $k a_{i} \in(0, \epsilon)$. Since the rotation of $S^{1}$ in $a_{i}$ is ergodic, $p_{i}(m, \epsilon) \rightarrow \epsilon$ as $m \rightarrow \infty$. Summing up over all $i=1, \ldots, r$, we conclude that the number of integers $k \in\left(k_{\epsilon}, m\right]$ with $k a_{i} \in(0, \epsilon)$ for at least one $i=1, \ldots, r$ does not exceed $\left(m-k_{\epsilon}\right) r \epsilon+o\left(m-k_{\epsilon}\right)$. Therefore, when $\epsilon<1 / r$ and $m$ is large enough, there exists an integer $k \in\left(k_{\epsilon}, m\right]$ such that $k a_{i} \notin(0, \epsilon)$ for all $i$. This contradicts Theorem 1.17.

Remark 3.2 We conjecture that $\lim \sup \left|\mathcal{S}_{\lambda_{0}}\left(H^{(k)}\right)\right|=\infty$ as $k \rightarrow \infty$, under the hypotheses of Proposition 3.1. It is easy to see that this is true when $\lambda_{0}=\infty$ and the argument above falls just short of showing that this is true in general.

Returning to the proof of Theorem 1.18, let us first assume that $\left.c_{1}(M)\right|_{\pi_{2}(M)}=0$. In this case, the mean index of a periodic orbit is independent of capping and we suppress the capping in the notation. By Theorem 1.17 and (LF2), for every sufficiently large $k$, the Hamiltonian $H$ has a $k$-periodic orbit $y$ with $\Delta_{H^{(k)}}(y) \in[1,2 n+1]$. When $k$ is prime, $y$ is either simple or the $k$-th iteration of a one-periodic orbit $z$ of $H$. The latter is impossible when $k$ is large, since for every one-periodic orbit $z$ either 
$\Delta_{H^{(k)}}\left(z^{k}\right) \equiv 0$ or $\left|\Delta_{H^{(k)}}\left(z^{k}\right)\right| \rightarrow \infty$ as $k \rightarrow \infty$, and there are only finitely many one-periodic orbits. (Observe also that here, in contrast with the general case, the assumption that $M$ is rational is used only to ensure that $M$ satisfies the requirements of Theorem 1.17, where it plays a purely technical role.)

Finally, the remaining case where $\left.\omega\right|_{\pi_{2}(M)}=0$ (ie, $\lambda_{0}=\infty$ ) is treated in a similar fashion, but with action used in place of the mean index. This is a standard argument $[20 ; 30]$.

\subsection{Proof of Theorem 1.1}

Here, as in $[20 ; 24 ; 30]$, we consider two cases: the case where $H$ has a symplectically degenerate maximum and the case where all one-periodic orbits of $H$ have nonzero mean index. The assertion in the former case follows from Theorem 1.18. In the latter case, since the mean index of an orbit is independent of capping, the support of local Floer homology of any periodic orbit shifts away from the interval $[0,2 n]$ as the order of iteration grows. Then the theorem follows again by the standard argument $[20 ; 30 ; 50]$.

\subsection{Proof of Theorem 1.3}

The theorem is an immediate consequence of the following result:

Proposition 3.3 Let $M$ be a closed, rational, weakly monotone manifold with $2 N>$ $3 n$ and let $H$ be a Hamiltonian on $M$ with finitely many geometrically distinct periodic orbits. Then $\left|\mathcal{I}^{\infty}(H)\right| \geq\lceil N / n\rceil$.

Proof of Proposition 3.3 Since $H$ has finitely many periodic orbits, the Hamiltonian $F=H^{\left(k_{0}\right)}$, for a suitably chosen iteration $k_{0}>0$, has the following properties:

- $\mathcal{I}_{2 N}\left(F^{(k)}\right) \subset k \mathcal{I}_{2 N}(F)$ for all $k>0$.

- $\mathcal{I}_{2 N}(F) \cap(\mathbb{Q} / \mathbb{Z})$ contains at most one point $\{0\}$, ie, whenever the mean index of a periodic orbit of $F$ is rational, this index is integer and divisible by $2 N$.

- $\left(\mathcal{I}_{2 N}(F)-\mathcal{I}_{2 N}(F)\right) \cap(\mathbb{Q} / \mathbb{Z})$ contains at most one point $\{0\}$, ie, whenever the difference of the mean indices of two periodic orbits of $F$ is rational, this difference is an integer divisible by $2 N$.

It suffices to show that $\left|\mathcal{I}^{\infty}(F)\right| \geq\lceil N / n\rceil$. For the sake of simplicity, let us assume again that $F$ is one-periodic (rather than $k_{0}$-periodic) - this can always be achieved via a change of time. 
We break the collection of capped one-periodic orbits of $F$ into two groups: those with integer mean indices (divisible by $2 N$ ) and the remaining capped orbits - those with irrational mean indices. These groups do not change when $F$ is replaced by any of its iterations $F^{(k)}$ due to the first condition in the description of $F$. By (LF2), for every $k$, there exists a $k$-periodic orbit $\bar{x}$ such that $\operatorname{HF}_{2 n}\left(F^{(k)}, \bar{x}\right) \neq 0$. We claim that $\bar{x}$ is necessarily in the second group, ie, $\Delta_{F^{(k)}}(\bar{x}) \notin 2 N \mathbb{Z}$. Indeed, otherwise, we would have $\Delta_{F^{(k)}}(\bar{x})=2 N l$ for some $l \in \mathbb{Z}$. By (LF5), the local Floer homology of $\bar{x}$ is supported in the interval $[2 N l, 2 N l+2 n]$. When $l \neq 0$, this interval does not contain $2 n$, and hence $\mathrm{HF}_{2 n}\left(F^{(k)}, \bar{x}\right)=0$. If $l=0$, the orbit $\bar{x}$ is a symplectically degenerate maximum of $F^{(k)}=H^{\left(k k_{0}\right)}$, and thus $H$ has infinitely many geometrically distinct periodic orbits by Theorem 1.18(i), which contradicts our basic assumption.

Let $\mathcal{I}_{2 N}(F) \backslash\{0\}=\left\{\Delta_{1}, \ldots, \Delta_{r}\right\}$. By our choice of $k_{0}$ (the last two conditions), $\left|\mathcal{I}^{\infty}(F)\right| \geq r$. Since $\Delta_{i}$ is irrational, the point $k \Delta_{i}$ enters the $\operatorname{arc}[n, 3 n] \subset S_{2 N}^{1}=$ $\mathbb{R} / 2 N \mathbb{Z}$ of length $2 n$ with probability $n / N$. To be more precise, for every $k>0$ the number of $j$ such that $1 \leq j \leq k$ and $j \Delta_{i} \in[n, 3 n]$ is equal to $k n / N$, up to a constant independent of $k$. As is observed above, for every $k>0$, at least one of the points $k \Delta_{1}, \ldots, k \Delta_{r}$ is within the $\operatorname{arc}[n, 3 n]$. Hence, $r \geq\lceil N / n\rceil$.

Remark 3.4 A minor modification of this argument shows that whenever $H$ has finitely many periodic orbits and $N \geq n+1$, the mean indices of the periodic orbits of $H$ must satisfy a resonance relation modulo $2 N$. The circle of questions concerning these resonance relations is explored in more detail in [27].

\section{The bounded gap theorem}

In this section, we establish the bounded gap theorem (Theorem 1.4), following closely the line of reasoning from $[20 ; 24]$ where a similar result is proved for symplectically aspherical manifolds. The proof of Theorem 1.4 relies on Theorem 1.17, established in Section 5, and on two properties of Floer homology: the stability of filtered Floer homology and the persistence of local Floer homology under iterations, which we discuss first.

\subsection{Stability of Floer homology}

The stability of filtered Floer homology asserts that the part of filtered Floer homology that is stable under perturbations of the action interval is also stable with respect to variations of the Hamiltonians; $\mathrm{cf}[3 ; 5 ; 24]$. 
To be more precise, consider Hamiltonians $K$ and $F$ on a closed weakly monotone symplectic manifold $M$. Set

$$
E^{+}=\int_{0}^{1} \max _{M} F_{t} d t \text { and } E^{-}=-\int_{0}^{1} \min _{M} F_{t} d t
$$

so that $\|F\|=E^{+}+E^{-}$is the Hofer energy of $F$. Furthermore, let

$$
E_{0}^{+}=\max \left\{E^{+}, 0\right\} \quad \text { and } \quad E_{0}^{-}=\max \left\{E^{-}, 0\right\} \quad \text { and } \quad E_{0}(F)=E_{0}^{+}+E_{0}^{-} .
$$

Note that $E_{0}^{ \pm}=E^{ \pm}$and $E_{0}=\|F\|$ when $F$ is normalized or, more generally, $\min _{M} F_{t} \leq 0 \leq \max _{M} F_{t}$.

Let $a<b$ be such that the end points of the intervals $(a, b)$ and $\left(a+E_{0}, b+E_{0}\right)$ are outside $\mathcal{S}(K)$ while the end points of $\left(a+E_{0}^{+}, b+E_{0}^{+}\right)$are outside $\mathcal{S}(K \# F)$. These requirements are met by almost all $a$ and $b$.

Furthermore, consider the following commutative diagram:

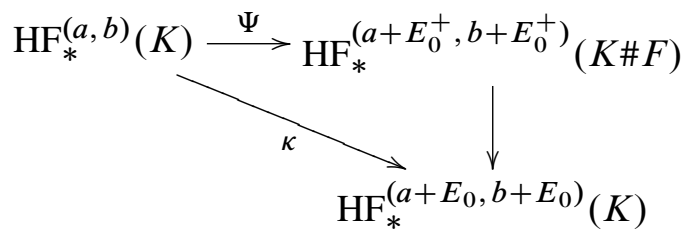

where $\kappa$ is the natural "quotient-inclusion" map, the homomorphism $\Psi$ is induced by the linear homotopy from $K$ to $K \# F$, and the vertical arrow is induced by the linear homotopy from $K \# F$ to $K$. We obviously have:

Lemma 4.1 (Stability of filtered Floer homology [24]) Assume that $\kappa(x) \neq 0$, where $x \in \mathrm{HF}_{*}^{(a, b)}(K)$. Then $\Psi(x) \neq 0$. In particular, $\mathrm{HF}_{*}^{\left(a+E_{0}^{+}, b+E_{0}^{+}\right)}(K \# F) \neq 0$, whenever $\kappa \neq 0$.

Remark 4.2 It is worth mentioning that the assertion of Lemma 4.1 is meaningful only when the length of the interval $(a, b)$ exceeds the size of the perturbation: if the intervals $(a, b)$ and $\left(a+E_{0}, b+E_{0}\right)$ do not overlap, the quotient-inclusion map $\kappa$ is necessarily zero.

\subsection{Persistence of local Floer homology}

The second ingredient needed in the proof of Theorem 1.4 is the persistence of local Floer homology under iterations. 
Let $M$ be an arbitrary symplectic manifold and let $x$ be an isolated one-periodic orbit of a Hamiltonian $H: S^{1} \times M \rightarrow \mathbb{R}$. Recall that a positive integer $k$ is an admissible iteration of $\varphi=\varphi_{H}$ (with respect to $x$ ) if $\lambda^{k} \neq 1$ for all eigenvalues $\lambda \neq 1$ of $d \varphi_{p}: T_{p} M \rightarrow T_{p} M$, where $p=x(0)$. In other words, $k$ is admissible if and only if $d \varphi_{p}^{k}$ and $d \varphi_{p}$ have the same generalized eigenvectors with eigenvalue one. For instance, when no eigenvalue $\lambda \neq 1$ is a root of unity (eg, all eigenvalues are equal to 1), all $k>0$ are admissible. It is easy to see that admissible iterations form a quasi-arithmetic sequence.

Theorem 4.3 (Persistence of local Floer homology [24]) Assume that $x$ is an isolated one-periodic orbit of $H$. Then the $k$-periodic orbit $x^{k}$ is also isolated, whenever $k$ is admissible, and the local Floer homology groups of $H$ and $H^{(k)}$ coincide up to a shift of degree:

$$
\mathrm{HF}_{*}\left(H^{(k)}, \bar{x}^{k}\right)=\mathrm{HF}_{*+s_{k}}(H, \bar{x}) \text { for some } s_{k} .
$$

Furthermore, $\lim _{k \rightarrow \infty} s_{k} / k=\Delta_{H}(\bar{x})$, provided that $\operatorname{HF}_{*}(H, \bar{x}) \neq 0$ and hence the shifts $s_{k}$ are uniquely determined by (4-1). Moreover, when $\Delta_{H}(\bar{x})=0$ and $\mathrm{HF}_{2 n}(H, \bar{x}) \neq 0$, the orbit $x$ is strongly degenerate, all $s_{k}$ are zero, and $\bar{x}$ is a symplectically degenerate maximum.

This theorem is proved in [24] under the additional assumption that $M$ is symplectically aspherical. However, as was already pointed out in that paper, the argument is essentially local and goes through for a general symplectic manifold. Namely, by the results of Section 2.3, the statement reduces to the case where $\bar{x}$ is a constant orbit $p$ with trivial capping. The rest of the proof concerns only the behavior of $H$ on a neighborhood of $p$ [24].

\subsection{Proof of Theorem 1.4}

Similarly to its predecessors from [20;24], the argument is based on the analysis of two cases. Namely, since $\mathrm{HF}_{2 n}(H) \neq 0$, there exists a capped one-periodic orbit $\bar{x}$ of $H$ with $\operatorname{HF}_{2 n}(H, \bar{x}) \neq 0$. Thus, $\Delta_{H}(\bar{x}) \geq 0$. The first, "degenerate", case is where $\Delta_{H}(\bar{x})=0$, ie, $\bar{x}$ is a symplectically degenerate maximum, while in the second, "nondegenerate", case $\Delta_{H}(\bar{x})>0$. (Since, in general, $\bar{x}$ is not unique, the two cases are not mutually exclusive for a given Hamiltonian $H$.)

4.3.1 The "degenerate" case: $\operatorname{HF}_{2 n}(H, \bar{x}) \neq 0$ and $\Delta_{H}(\bar{x})=0$ This is the case where $\bar{x}$ is a symplectically degenerate maximum of $H$. By Theorem 1.17, for every sufficiently small $\epsilon>0$ there exists an integer $k_{\epsilon}>0$ such that for all $k>k_{\epsilon}$ we have

$$
\mathrm{HF}_{2 n+1}^{(k c, k c+\epsilon)}\left(H^{(k)}\right) \neq 0,
$$


where $c=A_{H}(\bar{x})$. Hence, $H$ has a $k$-periodic orbit $\bar{z}_{k}$ with

$$
0<\mathcal{A}_{H^{(k)}}\left(\bar{z}_{k}\right)-\mathcal{A}_{H^{(k)}}\left(\bar{x}^{k}\right)<\epsilon
$$

and

$$
1 \leq \Delta_{H^{(k)}}\left(\bar{z}_{k}\right)-\Delta_{H^{(k)}}\left(\bar{x}^{k}\right) \leq 2 n+1 .
$$

Note that once $\epsilon$ is smaller than the rationality constant $\lambda_{0}$ of $M$, the orbit $\bar{z}_{k}$ cannot be a recapping of $\bar{x}^{k}$. It remains to set $v_{i}=k_{\epsilon}+i$ and $\bar{y}_{i}=\bar{z}_{k_{\epsilon}+i}$.

4.3.2 The "nondegenerate" case: $\operatorname{HF}_{2 n}(H, \bar{x}) \neq 0$ and $\Delta_{H}(x)>0$ In this case, $x$ is weakly nondegenerate [24]. Furthermore, it is easy to see that there exists an infinite, quasi-arithmetic sequence $l_{1}<l_{2}<\cdots$ of admissible iterations such that

$$
l_{i}+1<l_{i+1}
$$

and the local Floer homology jumps from $l_{i}$ to $l_{i+1}$, ie,

$$
0 \neq \operatorname{HF}_{*}\left(H^{\left(l_{i}\right)}, \bar{x}^{l_{i}}\right) \neq \operatorname{HF}_{*}\left(H^{\left(l_{i}+1\right)}, \bar{x}^{l_{i}+1}\right) .
$$

Here the group $\mathrm{HF}_{*}\left(H^{\left(l_{i}\right)}, \bar{x}^{l_{i}}\right)$ is nontrivial by Theorem 4.3 and, by (LF5), supported in the interval $\left(l_{i} \Delta_{H}(\bar{x}), l_{i} \Delta_{H}(\bar{x})+2 n\right)$, while the group $\operatorname{HF}_{*}\left(H^{\left(l_{i}+1\right), x^{l_{i}+1}}\right)$ may possibly be trivial.

Let us normalize $H$ by requiring it to have zero mean. Then $E_{0}=\|H\|$ as has been pointed out above. Set $c=\mathcal{A}_{H}(\bar{x})$. Thus, $\mathcal{A}_{H^{(k)}}\left(\bar{x}^{k}\right)=k c$ for all $k$. Recall also that, by the hypotheses of the theorem, we have

(i) $E_{0}=\|H\|<\lambda_{0}$, where $\lambda_{0}>0$ is the rationality constant of $M$, or

(ii) $N \geq 2 n$, where $N$ is the minimal Chern number of $M$.

Fix $\alpha>E_{0}>0$ and $\beta>0$. When condition (i) is satisfied, we also require that

$$
\max \left\{\alpha, \beta+E_{0}\right\}<\lambda_{0} .
$$

The $i$-th entry in the sequence $v_{i}$ is equal either to $l_{i}$ or to $l_{i}+1$. Assume that $v_{1}, \ldots, v_{i-1}$ and the periodic orbits $\bar{y}_{1}, \ldots, \bar{y}_{i-1}$ have been chosen. Our goal is then to find a $v_{i}$-periodic orbit $\bar{y}=\bar{y}_{i}$ with either $v_{i}=l_{i}$ or $v_{i}=l_{i}+1$ satisfying the requirements of Theorem 1.4. (The first orbit $y_{1}$ and the period $v_{1}$ are chosen in a similar fashion.) Note that, since $x$ is one-periodic, $\bar{y}_{i}$ and $\bar{x}^{v_{i}}$ are geometrically distinct if and only if $y_{i} \neq x^{v_{i}}$, ie, $\bar{y}_{i}$ is not a recapping of $\bar{x}^{v_{i}}$.

Set $l=l_{i}$ to simplify the notation, and $a=l c-\alpha$ and $b=l c+\beta$. Fix $m$ such that $\operatorname{HF}_{m}\left(H^{(l)}, \bar{x}^{l}\right) \neq 0$, but $\operatorname{HF}_{m}\left(H^{(l+1)}, \bar{x}^{l+1}\right)=0$. Under the above assumptions, $\bar{y}_{i}$ and $v_{i}$ are chosen differently in each of the following three cases. 
Case $1 \mathrm{HF}_{m}^{(a, b)}\left(H^{(l)}\right)=0$. It is easy to see that in this case $H$ has an $l$-periodic orbit $\bar{y}$ which kills the contribution of $\operatorname{HF}_{m}\left(H^{(l)}, \bar{x}^{l}\right)$ to $\mathrm{HF}_{m}^{(a, b)}\left(H^{(l)}\right)$, such that $\left|\Delta_{H^{(l)}}(\bar{y})-m\right| \leq n+1$ and the action $\mathcal{A}_{H^{(l)}}(\bar{y}) \neq k c$ is in the interval $(a, b)$. It is clear that the action and mean index gaps for $\bar{x}^{l}$ and $\bar{y}$ are bounded from above by $\max \{\alpha, \beta\}$ and, respectively, $2 n+1$ and the action gap is strictly positive. Hence, in particular, $\bar{y} \neq \bar{x}^{k}$.

If condition (i) is satisfied, $\bar{y}$ is not a nontrivial recapping of $\bar{x}^{l}$ due to (4-3). Under condition (ii), the same is true since the mean index gap does not exceed $2 n+1$ which is smaller than $2 N$. (Here it would be sufficient to assume $N>n$ in (ii).) Thus $y \neq x^{l}$. We set $v_{i}=l$ and $\bar{y}_{i}=\bar{y}$.

Case $2 \mathrm{HF}_{m}^{\left(a+E_{0}^{+}, b+E_{0}^{+}\right)}\left(H^{(l+1)}\right) \neq 0$. In this case, there exists an $(l+1)$-periodic orbit $\bar{y}$ with action in the interval $\left(a+E_{0}^{+}, b+E_{0}^{+}\right)$and $\mathrm{HF}_{m}\left(H^{(l+1)}, \bar{y}\right) \neq 0$. We set $v_{i}=l+1$ and $\bar{y}_{i}=\bar{y}$. To verify the requirements of the theorem, we first note that

$$
\left|\mathcal{A}_{H^{(l+1)}}\left(\bar{x}^{l+1}\right)-\mathcal{A}_{H^{(l+1)}}(\bar{y})\right| \leq \max \left\{\alpha-E_{0}^{+}, \beta+E_{0}^{+}\right\} .
$$

Furthermore, since $\left|\Delta_{H^{(l+1)}}(\bar{y})-m\right| \leq n$ and $\left|\Delta_{H^{(l)}}\left(\bar{x}^{l}\right)-m\right| \leq n$, we have

$$
\left|\Delta_{H^{(l+1)}}\left(\bar{x}^{l+1}\right)-\Delta_{H^{(l+1)}}(\bar{y})\right| \leq \Delta_{H}(\bar{x})+2 n<4 n .
$$

The last inequality in (4-5) follows from the fact that $\operatorname{HF}_{2 n}(H, \bar{x}) \neq 0$, and hence, by (LF5), $\Delta_{H}(x)<2 n$ since $x$ is weakly nondegenerate. The inequalities (4-4) and (4-5) give upper bounds on the action and mean index gaps.

To show that $y \neq x^{l+1}$, ie, $\bar{y}$ is not a recapping of $\bar{x}^{l+1}$, we argue as follows. First note that $\bar{y} \neq \bar{x}^{l+1}$, since $\mathrm{HF}_{m}\left(H^{(l+1)}, \bar{x}^{l+1}\right)=0$ while $\mathrm{HF}_{m}\left(H^{(l+1)}, \bar{y}\right) \neq 0$. Thus, it remains to prove that $\bar{y}$ cannot be a nontrivial recapping of $\bar{x}^{l+1}$. When condition (i) is satisfied this follows immediately from (4-3) and (4-4). If condition (ii) holds, this follows from (4-5).

Case $3 \mathrm{HF}_{m}^{(a, b)}\left(H^{(l)}\right) \neq 0$, but $\mathrm{HF}_{m}^{(a+E, b+E)}\left(H^{(l+1)}\right)=0$. First note that throughout the entire argument we might have assumed that $H_{0} \equiv 0 \equiv H_{1}$, and thus $H^{\# k}$ is also one-periodic for all $k$. Now we can identify one-periodic orbits of $H^{\# k}$ with $k$-periodic orbits of $H$ and the Floer homology of $H^{\# k}$ and of $H^{(k)}$. Using stability of filtered Floer homology as in Section 4.1 with $K=H^{\# l}$ and $F=H$, we see that the quotient-inclusion map

$$
\kappa: \mathrm{HF}_{m}^{(a, b)}\left(H^{\# l}\right) \rightarrow \mathrm{HF}_{m}^{(a+E, b+E)}\left(H^{\# l}\right)
$$


is necessarily zero, for $\mathrm{HF}_{m}^{\left(a+E_{0}^{+}, b+E_{0}^{+}\right)}\left(H^{\#(l+1)}\right)=0$. Since $\operatorname{HF}_{m}^{(a, b)}\left(H^{\# l}\right) \neq 0$, we infer by a simple exact sequence argument that

or/and

$$
\begin{aligned}
& \operatorname{HF}_{m}^{(a, a+E)}\left(H^{(l)}\right)=\operatorname{HF}_{m}^{(a, a+E)}\left(H^{\# l}\right) \neq 0 \\
& \mathrm{HF}_{m+1}^{(b, b+E)}\left(H^{(l)}\right)=\mathrm{HF}_{m+1}^{(b, b+E)}\left(H^{\# l}\right) \neq 0 .
\end{aligned}
$$

In the former case, there exists an $l$-periodic orbit $\bar{y}$ with action in the range $(a, a+E)$ and $\left|m-\Delta_{H^{(l)}}(\bar{y})\right| \leq n$. In the latter case, there exists an $l$-periodic orbit $\bar{y}$ with action in the range $(b, b+E)$ and $\left|m+1-\Delta_{H^{(l)}}(\bar{y})\right| \leq n$. Then

$$
0<\min \{\alpha-E, \beta\}<\left|\mathcal{A}_{H^{(l)}}(\bar{y})-\mathcal{A}_{H^{(l)}}\left(\bar{x}^{l}\right)\right| \leq \max \{\alpha, \beta+E\}
$$

and

$$
\left|\Delta_{H^{(l)}}\left(\bar{x}^{l}\right)-\Delta_{H^{(l)}}(\bar{y})\right| \leq 2 n+1 .
$$

Since the action gap is positive, $\bar{y} \neq \bar{x}^{l}$. Furthermore, when (i) holds, $\bar{y}$ is not a nontrivial recapping $\bar{x}^{l}$ by (4-3) and (4-6). If condition (ii) is satisfied, $\bar{y}$ is not a nontrivial recapping $\bar{x}^{l}$ since the mean index gap does not exceed $2 n+1<2 N$. (Here, as in Case 1, it would be sufficient to assume that $N>n$.) Thus $y \neq x^{l}$. We set $v_{i}=l$ and $\bar{y}_{i}=\bar{y}$.

This completes the proof of Theorem 1.4.

Remark 4.4 It is easy to infer from the proof of the theorem that the upper bound on the action gap is in fact $E_{0}=\|H\|$ when $\alpha$ and $\beta$ satisfy (4-3), for $\beta>0$ and $\alpha-E_{0}>0$ can be made arbitrarily small, and the upper bound on the mean index gap is $4 n$.

\subsection{The action-index gap and the Conley conjecture}

In this section, we will briefly discuss the relation between Theorem 1.4 and the Conley conjecture.

As is pointed out in Section 1.3, the hypotheses of Theorem 1.4 are automatically satisfied for any Hamiltonian $H$ with isolated periodic orbits when $M$ is symplectically aspherical. In this case the theorem was proved in [24] in a stronger form. Namely, the sequence $v_{i}$ can be chosen to be a subsequence of any quasi-arithmetic sequence of admissible iterations and the action-index gap (1-1) is nonzero. With these two amendments - the second one is particularly important - the theorem readily implies the Conley conjecture for symplectically aspherical manifolds [24].

However, to the best of our understanding, once $N<\infty$ and/or $\lambda_{0}<\infty$, Theorem 1.4 , even if it were proved in this stronger form, would not directly imply the Conley 
conjecture in the cases where it is expected to hold, eg, for manifolds with large $N$. For instance, we have been unable to infer Theorem 1.1 from Theorem 1.4. Furthermore, even though the assertion of Theorem 1.7 is at least as strong as the aspherical counterpart of Theorem 1.4, the Conley conjecture fails in general under the hypotheses of Theorem 1.7 as becomes clear by comparing Example 1.2 and Example 1.10. Yet, Theorems 1.4 and 1.7 yield a criterion for the existence of infinitely many periodic orbits; see Corollary 1.11 .

\section{Periodic orbits in the presence of a symplectically degener- ate maximum}

The main objective of this section is to prove Theorem 1.17. The proof follows the same line of reasoning as the argument from [20] where the theorem is established for symplectically aspherical manifolds. The idea of the proof is the squeezing trick [4; 26; 35]: the Hamiltonian $H$ is bounded from above and below by functions $H_{ \pm}$such that the monotone homotopy map for the iterated Hamiltonians $H_{ \pm}^{(k)}$ is nonzero for the action interval in question. Since this map factors through the Floer homology of $H^{(k)}$, the latter Floer homology group is also nontrivial.

The key to realizing this idea is a geometrical characterization of symplectically degenerate maxima, building on the results of $[20 ; 24 ; 30]$. Namely, we show that a Hamiltonian diffeomorphism $\varphi_{H}$ with a symplectically degenerate maximum $\bar{x}$ can be generated by a Hamiltonian $K$ with a strict local maximum at $p=x(0)$, for all times, and with arbitrarily small Hessian at $p$; see Proposition 5.8. Moreover, $\varphi_{H}=\varphi_{K}$ in the universal covering of the group of Hamiltonian diffeomorphisms, and hence $H$ and $K$ have the same filtered Floer homology. The problem is thus reduced to proving the theorem for a Hamiltonian $H=K$ with a genuine maximum at $\bar{x}=p$. Then the construction of $H_{ \pm}$and the analysis of the monotone homotopy map can be carried out by fairly standard methods.

The main difference between the proof of Theorem 1.17 given below and the argument for symplectically aspherical manifolds is that in the latter case the Floer homology in question and the monotone homotopy map can be determined explicitly [20; 26] while in the setting of Theorem 1.17 such a calculation looks problematic. To circumvent this difficulty, we isolate a direct summand in the Floer homology groups of $H_{ \pm}$which behaves exactly as if the manifold $M$ were symplectically aspherical, eg, $\mathbb{R}^{2 n}$. As a consequence, the monotone homotopy map between the corresponding summands for $H_{ \pm}$is nonzero. This procedure is of rather general nature and independent interest and is described in detail in the next section. 


\subsection{Direct sum decomposition of filtered Floer homology}

Throughout this section, we assume that $M$ is weakly monotone and geometrically bounded and rational; see Audin and Lafontaine [2] and Cieliebak, Ginzburg and Kerman [6] for the definition and a detailed discussion of geometrically bounded manifolds. Let $U \subset W \subset M$ be open sets with smooth boundary and compact closure. For the sake of simplicity, we will assume that the closed domains $\bar{U}$ and $\bar{W}$ are isotopic in $M$, eg, $W$ is a small neighborhood of $\bar{U}$. Consider a Hamiltonian $F$, which is constant on $M \backslash U$, and set $C:=\left.F\right|_{M \backslash U}$. Let $(a, b)$ be an interval such that

$$
a \text { and } b \text { are outside } \mathcal{S}(F) \text { and }(a, b) \cap\left(C+\lambda_{0} \mathbb{Z}\right)=\varnothing,
$$

where $\lambda_{0}$ is the rationality constant. (The set $C+\lambda_{0} \mathbb{Z}$ is formed by action values of $F$ on trivial orbits outside $U$ with arbitrary cappings.) For instance, if $\left.\omega\right|_{\pi_{2}(M)}=0$, the second part of (5-1) simply asserts that $C \notin(a, b)$. Note that under these conditions the Floer homology $\operatorname{HF}_{*}^{(a, b)}(F)$ is defined (see Remark 2.2) and that (5-1) implies, in particular, that $b-a<\lambda_{0}$.

Assume now that all one-periodic orbits of $F$ with action in $(a, b)$ are nondegenerate. Denote by $\mathrm{CF}_{*}^{(a, b)}(F ; U)$ the vector space (over $\mathbb{F}$ ) generated by such orbits with cappings contained in $U$ or, more precisely, with cappings equivalent to those contained in $U$. (Since $W \supset U$ is isotopic to $U$, we can equivalently require the cappings to be contained in $W$.) In particular, the orbits in question must also be contained in $U$. Let $\mathrm{CF}_{*}^{(a, b)}(F ; M, U)$ be the vector space generated by the remaining capped one-periodic orbits with action in the interval $(a, b)$. (These orbits, but not their cappings, are also contained in $U$ by (5-1).) Thus, we have the direct sum decomposition

$$
\mathrm{CF}_{*}^{(a, b)}(F)=\mathrm{CF}_{*}^{(a, b)}(F ; U) \oplus \mathrm{CF}_{*}^{(a, b)}(F ; M, U)
$$

on the level of vector spaces. Fix now an arbitrary, not necessarily $F$-regular, almost complex structure $J_{0}$ compatible with $\omega$.

Lemma 5.1 There exists a constant $\epsilon(U, W, M)>0$, independent of $F$, such that whenever $b-a<\epsilon(U, W, M)$ the decomposition (5-2) is a direct sum of complexes for any $F$-regular almost complex structure $J$ sufficiently close to $J_{0}$ and compatible with $\omega$. Thus, we have, in the obvious notation, the decomposition of Floer homology

$$
\mathrm{HF}_{*}^{(a, b)}(F)=\mathrm{HF}_{*}^{(a, b)}(F ; U) \oplus \mathrm{HF}_{*}^{(a, b)}(F ; M, U) .
$$

The direct sum decompositions (5-2) and (5-3) are preserved by the long exact sequence maps, provided that all action intervals are shorter than $\epsilon(U, W, M)$, and by monotone decreasing homotopy maps, as long as the Hamiltonians are constant on $M \backslash U$. 
Note that the constant $\epsilon(U, W, M)$ also depends on $J_{0}$ even though this dependence is suppressed in the notation. However, $\epsilon(U, W, M)$ is bounded away from zero when $J_{0}$ varies within a compact set. Furthermore, as is clear from the proof of the lemma, the constant $\epsilon(U, W, M)$ is completely determined by the restriction of $J_{0}$ to $\bar{W} \backslash U$.

The proof of Lemma 5.1 is essentially the standard argument that a Floer connecting trajectory with small energy connecting orbits from $\mathrm{CF}_{*}^{(a, b)}(F ; U)$ must lie in $W$, based on the Gromov compactness theorem. Postponing the proof, let us show how the lemma extends, essentially by continuity, to degenerate Hamiltonians; cf Remark 2.2. Observe first that, as readily follows from the last assertion of the lemma, the decomposition (5-3) is independent of $J$ and, in fact, of $J_{0}$ when $J_{0}$ varies within a fixed compact set and $b-a$ is sufficiently small.

Let $F$ be an arbitrary Hamiltonian which is constant on $M \backslash U$ and meets the requirement of (5-1). Due to the latter condition, nondegeneracy is a generic requirement on the Hamiltonian. More precisely, $F$ can be $C^{2}$-approximated by Hamiltonians $\widetilde{F}$ satisfying (5-1) and such that all one-periodic orbits of $\widetilde{F}$ with action in $(a, b)$ are nondegenerate. Fix $J_{0}$ and set, assuming that $b-a<\epsilon(U, W, M)$,

and

$$
\begin{aligned}
\mathrm{HF}_{*}^{(a, b)}(F ; U) & =\mathrm{HF}_{*}^{(a, b)}(\widetilde{F} ; U) \\
\mathrm{HF}_{*}^{(a, b)}(F ; M, U) & =\mathrm{HF}_{*}^{(a, b)}(\widetilde{F} ; M, U) .
\end{aligned}
$$

Clearly, these groups are independent of $\widetilde{F}$ and $J$, when $\widetilde{F}$ is $C^{2}$-close to $F$ and $J$ is close to $J_{0}$. As an immediate consequence of Lemma 5.1, we obtain:

Proposition 5.2 There exists a constant $\epsilon(U, W, M)>0$, independent of $F$, such that the direct sum decomposition (5-3) holds and is preserved by the long exact sequence and decreasing monotone homotopy maps, as long as all action intervals are shorter than $\epsilon(U, W, M)$ and the Hamiltonians are constant on $M \backslash U$.

Remark 5.3 It is essential that in the last assertion of the proposition and of the lemma where a monotone decreasing homotopy $F^{s}$ is considered, the constant value $C_{s}=$ $\left.F^{s}\right|_{M \backslash U}$ may depend on $s$ and condition (5-1) is imposed only on the Hamiltonians $F^{0}$ and $F^{1}$, but not on all Hamiltonians $F^{s}$. In particular, the set $C_{s}+\lambda_{0} \mathbb{Z}$ is not required to have trivial intersection with the interval $(a, b)$ for all $s \in[0,1]$, but only for $s=0$ and $s=1$.

Proof of Lemma 5.1 The set $Z=\bar{W} \backslash U$ is a "shell" bounded by $S_{0}=\partial \bar{U}$ and $S_{1}=\partial \bar{W}$. Let $J$ be an almost complex structure sufficiently close to $J_{0}$. Consider $J$-holomorphic curves $v: \Sigma \rightarrow Z$ with boundary on $S_{0} \cup S_{1}=\partial Z$ such that $v(\Sigma) \cap S_{0}$ 
and $v(\Sigma) \cap S_{1}$ are both nonempty. By the Gromov compactness theorem, there exists a constant $\epsilon(U, W, M)>0$ such that

$$
\int_{v} \omega>\epsilon(U, W, M)
$$

for all $v$ and all $J$ close to $J_{0}$.

Let $\bar{x}$ and $\bar{y}$ be two capped one-periodic orbits of $F$ with action in $(a, b)$, connected by a Floer antigradient trajectory $u$, and such that one of these orbits, say $\bar{x}$, has capping in $U$. To prove that (5-2) is a direct sum of complexes, we need to show that the capping of the second orbit $\bar{y}$ is also in $U$, provided that $b-a<\epsilon(U, W, M)$. To this end, it suffices to prove that $u$ is contained in $W$, for then the capping of $\bar{y}$ is homotopic to one contained in $U$.

The difference of actions $\left|\mathcal{A}_{F}(\bar{x})-\mathcal{A}_{F}(\bar{y})\right|$ is equal to the energy

$$
E(u)=\int_{-\infty}^{\infty} \int_{S^{1}}\left\|\partial_{s} u\right\|^{2} d t d s .
$$

Note that the part of $u$ that is not contained in $U$ is a holomorphic curve in $M$ with boundary on $S_{0}=\partial \bar{U}$. Let $\Sigma \subset \mathbb{R} \times S^{1}$ be the collection of all $(s, t)$ such that $u(s, t) \in Z$. Without loss of loss of generality, we may assume that $u$ is transverse to $S_{0}$ and $S_{1}$, by perturbing these hypersurfaces slightly, and thus $\Sigma$ is a closed domain in $\mathbb{R} \times S^{1}$ with smooth boundary. Clearly, $v:=\left.u\right|_{\Sigma}$ is a holomorphic curve with values in $Z$ and boundary in $\partial Z$. Thus,

$$
\epsilon(U)>b-a \geq E(u) \geq \int_{\Sigma}\left\|\partial_{s} u\right\|^{2} d t d s=\int_{\Sigma} v .
$$

Arguing by contradiction, assume that $u$ is not entirely contained in $W$. Then $v(\Sigma) \cap S_{0}$ and $v(\Sigma) \cap S_{1}$ are nonempty. Hence, the upper bound (5-4) holds, which is impossible by $(5-5)$.

The fact that the decomposition (5-3) is preserved by the exact sequence maps is a formal consequence of the definitions and of the complex decomposition (5-2).

The proof of the assertion that (5-3) is preserved by the monotone homotopy maps is identical to the above argument. (The only modification is that, when $u$ is a homotopy connecting trajectory, we have the inequality $\left|\mathcal{A}_{F}(\bar{x})-\mathcal{A}_{F}(\bar{y})\right| \geq E(u)$ rather than an equality.)

Furthermore, the proof of Lemma 5.1 yields:

Proposition 5.4 The Floer homology $\mathrm{HF}_{*}^{(a, b)}(F ; U)$ and the monotone homotopy maps are independent of the ambient manifold $M$ when $b-a$ is sufficiently small. 
More precisely, this proposition should be read as follows. Let a closed symplectic manifold $\bar{W}$ with boundary be embedded as a closed domain into weakly monotone, rational, geometrically bounded symplectic manifolds $M_{1}$ and $M_{2}$. Assume that $F$ is a Hamiltonian on $\bar{W}$, constant on $\bar{W} \backslash U$. Thus, $F$ extends naturally to $M_{1}$ and to $M_{2}$ as a function constant outside $U$. Furthermore, assume that (5-1) is satisfied for both $M_{1}$ and $M_{2}$, and $b-a<\min \left\{\epsilon\left(U, W, M_{1}\right), \epsilon\left(U, W, M_{2}\right)\right\}$. Then, there is a canonical isomorphism between the groups $\operatorname{HF}_{*}^{(a, b)}(F ; U)$ for the two ambient manifolds $M_{1}$ and $M_{2}$. Furthermore, $\epsilon\left(U, W, M_{1}\right)=\epsilon\left(U, W, M_{2}\right)$ when the complex structure on $M_{1}$ and the complex structure on $M_{2}$ coincide on $\bar{W} \backslash U$.

Example 5.5 Assume that $M$ is symplectically aspherical. Then, regardless of the length of the interval $(a, b)$, by the very definition of $\mathrm{CF}_{*}^{(a, b)}(F ; U)$ we have $\mathrm{CF}_{*}^{(a, b)}(F)=\mathrm{CF}_{*}^{(a, b)}(F ; U)$ and $\mathrm{HF}_{*}^{(a, b)}(F)=\mathrm{HF}_{*}^{(a, b)}(F ; U)$ if $(5-1)$ is satisfied. However, this homology group depends on $M$, unless $b-a$ is small.

Example 5.6 Assume that $\bar{W}$ is a Darboux ball embedded in $M$ and $F$ is as above. Then, combining Example 5.5 with Proposition 5.4, we conclude that $\mathrm{HF}_{*}^{(a, b)}(F ; U)$, when $b-a$ is sufficiently small, is equal to the filtered Floer homology of $F$ viewed as a Hamiltonian on $\mathbb{R}^{2 n}$. In particular, when $M$ itself is symplectically aspherical, we obtain equality between the filtered Floer homology of $F$ on $M$ and $\mathbb{R}^{2 n}$ for small action intervals. (This observation provides a shortcut to the calculation of the Floer homology in [20, Section 7] reducing the problem to the case of functions on $\mathbb{R}^{2 n}$, analyzed in [26].) In the proof of Theorem 1.17, Propositions 5.2 and 5.4 are utilized in a similar fashion.

Remark 5.7 The proof of Lemma 5.1 also shows that $\mathrm{CF}_{*}^{(a, b)}(F ; U)$ equipped with the usual Floer differential is a complex with well-defined homology even when $M$ is not geometrically bounded; cf [25].

\subsection{Geometrical characterization of symplectically degenerate maxima}

In this section, we give a geometrical characterization of symplectically degenerate maxima, following [20; 24].

Recall that the norm $\|v\|_{\Xi}$ of a tensor $v$ on a finite-dimensional vector space $V$ with respect to a basis $\Xi$ is, by definition, the norm of $v$ with respect to the Euclidean inner product for which $\Xi$ is an orthonormal basis. For instance, let $A: V \rightarrow V$ be a linear map with all eigenvalues equal to zero. Then $\|A\|_{\Xi}$ can be made arbitrarily small by suitably choosing $\Xi$. In other words, for any $\sigma>0$, there exists $\Xi$ such that $\|A\|_{\Xi}<\sigma$. (Indeed, in some basis, $A$ is given by an upper triangular matrix with zeros 
on the diagonal; $\Xi$ is then obtained by appropriately scaling the elements of this basis.) When $\xi$ is a coordinate system near $p \in M$, we denote by $\xi_{p}$ the natural coordinate basis in $T_{p} M$ arising from $\xi$.

Proposition 5.8 Assume that a capped one-periodic orbit $\bar{x}$ of $H$ is a symplectically degenerate maximum. Fix a point $p \in M$, eg, $p=x(0)$. Then there exists a sequence of contractible loops $\eta_{i}$ of Hamiltonian diffeomorphisms generated by Hamiltonians $G^{i}$ such that $\Phi_{G^{i}}(p)=\bar{x}$, ie, $\eta_{i}$ sends $p$ with trivial capping to $\bar{x}$, and the following conditions are satisfied:

(K1) The point $p$ is a strict local maximum of $K_{t}^{i}$ for all $t \in S^{1}$ and all $i$, where $K^{i}$ is given by $H=G^{i} \# K^{i}$.

(K2) There exist symplectic bases $\Xi^{i}$ in $T_{p} M$ such that

$$
\left\|d^{2}\left(K_{t}^{i}\right)_{p}\right\|_{\Xi^{i}} \rightarrow 0 \text { uniformly in } t \in S^{1} .
$$

(K3) The linearization of the loop $\eta_{i}^{-1} \circ \eta_{j}$ at $p$ is the identity map for all $i$ and $j$ (ie, $d\left(\left(\eta_{i}^{t}\right)^{-1} \circ \eta_{j}^{t}\right)_{p}=I$ for all $\left.t \in S^{1}\right)$ and, moreover, the loop $\left(\eta_{i}^{t}\right)^{-1} \circ$ $\eta_{j}^{t}$ is contractible to id in the class of loops fixing $p$ and having the identity linearization at $p$.

Remark 5.9 As is easy to see, this proposition gives, in fact, a necessary and sufficient condition for $\bar{x}$ to be a symplectically degenerate maximum. Moreover, requiring (K1) and (K2) is already sufficient, and thus (K3) is a formal consequence of these two conditions [24, Remark 5.5].

Proof By Proposition 2.9, we may assume without loss of generality that $\bar{x}$ is already a constant orbit $p$ of $H$ with trivial capping. Then, the existence of local loops $\eta_{i}$ generated by Hamiltonians $G_{i}$ defined on neighborhoods of $p$ is a consequence of [24, Propositions 5.3 and 5.4] and Remark 5.9; see also [20, Proposition 4.5]. The loops $\eta_{i}$ have zero Maslov index. To see this, first note that by (K3) all loops $\eta_{i}$ have the same Maslov index. Furthermore, by (MI7),

$$
2 \mu_{G^{i}}(p)+\Delta_{K^{i}}(p)=\Delta_{H}(p)=0 .
$$

Here, $\mu_{G^{i}}(p) \in \mathbb{Z}$ and $\Delta_{K^{i}}(p) \rightarrow 0$ as $i \rightarrow \infty$ by (K2). Thus, $\mu_{G^{i}}(p)=0$. It follows now from Proposition 2.10 and Remark 2.11 that the local loops $\eta_{i}$ extend to global loops with required properties. 


\subsection{Proof of Theorem 1.17}

By Proposition 5.8, it is sufficient to prove the theorem for the Hamiltonian $K^{1}$ in place of $H$ and the constant orbit $p$ of $K^{1}$ with trivial capping in place of $\bar{x}$. Thus, keeping the notation $H$ for the Hamiltonian $K^{1}$, we may assume throughout the proof that

- the point $p$ is a strict local maximum of $H_{t}$ for all $t \in S^{1}$ and

- $d\left(\eta_{i}^{t}\right)_{p}=I$ for all $t \in S^{1}$.

Furthermore, without loss of generality, we may assume that $H \geq 0$ and $c=H_{t}(p)$ is independent of $t$.

Our goal is to construct functions $H_{ \pm}$such that $H_{+} \geq H \geq H_{-}$and the monotone homotopy map

$$
\Psi: \operatorname{HF}_{2 n+1}^{\left(k c+\delta_{k}, k c+\epsilon\right)}\left(H_{+}^{(k)}\right) \rightarrow \operatorname{HF}_{2 n+1}^{\left(k c+\delta_{k}, k c+\epsilon\right)}\left(H_{-}^{(k)}\right)
$$

is nonzero, provided that $k$ is large enough; see Figure 1. Then, since this map factors through $\mathrm{HF}_{2 n+1}^{\left(k c+\delta_{k}, k c+\epsilon\right)}\left(H^{(k)}\right)$, the latter group is also nontrivial.

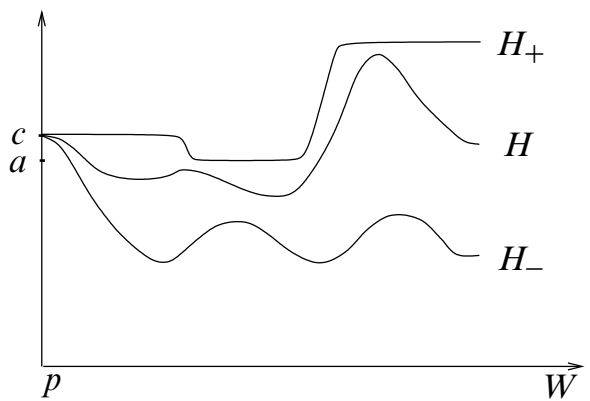

Figure 1: The functions $H$ and $H_{ \pm}$

5.3.1 The functions $H_{ \pm}$Fix a system of canonical coordinates on small Darboux balls $U \Subset W$ centered at $p$ and let the function $\rho$ on $W$ be the square of the distance to $p$ with respect to this coordinate system. The neighborhood $W$ is required to be so small that $c=H(p)$ is a strict global maximum of $H$ on $W$ and $W$ is contained in a larger Darboux ball. Fix also a compatible with $\omega$ almost complex structure $J_{0}$ on $M$ coinciding with the standard complex structure on a neighborhood of $\bar{W}$. In this case, as was pointed out in Section 5.1, $\epsilon(U, W, M)=\epsilon\left(U, W, \mathbb{R}^{2 n}\right)$. The parameter $\epsilon>0$ from the assertion of the theorem is required to satisfy

$$
\epsilon<\epsilon(U, W, M)=\epsilon\left(U, W, \mathbb{R}^{2 n}\right) .
$$


Pick four balls centered at $p$ in $U$ :

$$
B_{r_{-}} \subset B_{r} \subset B_{R} \subset B_{R_{+}} \Subset U .
$$

Let $H_{+}$be a function of $\rho$, also treated as a function on $U$, with the following properties (see Figure 1):

- $H_{+} \geq H$,

- $\left.H_{+}\right|_{B_{r_{-}}} \equiv c=H(p)$,

- on the shell $B_{r} \backslash B_{r_{-}}$the function $H_{+}$is monotone decreasing,

- $H_{+} \equiv a$ on the shell $B_{R} \backslash B_{r}$,

- $H_{+}$is monotone increasing on the shell $B_{R_{+}} \backslash B_{R}$,

- on $U \backslash B_{R_{+}}$, the function $H_{+}$is constant and equal to max $H_{+}$.

Furthermore, we extend $H_{+}$to $M$ by setting it to be constant and equal to max $H_{+}$ on the complement of $U$. The constant max $H_{+}$is chosen so that $H \leq H_{+}$on $M$ and $\max H_{+}>c+\epsilon$.

The precise description of $H_{+}$and the choice of the parameters in its construction are not explicitly used in this proof. It is essential though that the function $H_{+}$is defined exactly as in [20, Section 7]. At this stage, let us fix $H_{+}$.

Assume that $k$, depending on $H_{+}$, is sufficiently large and $\delta_{k}>0$, depending on $H_{+}$ and $k$, is sufficiently small. Fix $k$ and $\delta_{k}$.

Then the Hamiltonian $H_{-} \geq 0$, depending on $\mathrm{H}^{+}$and $k$, is constructed as follows. Recall that there exist

- a loop $\eta^{t}=\eta_{i}^{t}, t \in S^{1}$, of Hamiltonian diffeomorphisms fixing $p$ and

- a system of canonical coordinates $\xi=\xi^{i}$ on a small neighborhood $V \subset U$ of $p$

such that the Hamiltonian $K=K^{i}$ generating the flow $\left(\eta^{t}\right)^{-1} \circ \varphi_{H}^{t}$ has strict local maximum at $p$ and $\max _{t}\left\|d^{2}\left(K_{t}\right)_{p}\right\|_{\xi_{p}} \rightarrow 0$ as $i \rightarrow \infty$. Moreover, the loop $\eta$ has identity linearization at $p$, ie, $d\left(\eta^{t}\right)_{p}=I$ for all $t \in S^{1}$, and is contractible to id in the class of such loops. Let $\eta_{s}$ be a homotopy from $\eta$ to id such that $d\left(\eta_{s}^{t}\right)_{p} \equiv I$ and let $G_{t}^{s}$ be the one-periodic Hamiltonian generating $\eta_{s}^{t}$ and normalized by $G_{t}^{s}(p) \equiv 0$. The condition $d\left(\eta_{s}^{t}\right)_{p}=I$ is equivalent to $d^{2}\left(G_{t}^{s}\right)_{p}=0$. We normalize $K$ by requiring that $K_{t}(p) \equiv c$ or, equivalently, by $H=G \# K$.

When $i$ is sufficiently large and hence $k \max _{t}\left\|d^{2}\left(K_{t}\right)_{p}\right\|_{\xi_{p}}$ is sufficiently small, there exists a "bump function" $F$ (with respect to the coordinate system $\xi$ ) on a neighborhood $V \subset B_{r_{-}}$of $p$ such that 
- $k\left\|d^{2} F_{p}\right\|_{\xi_{p}}$ is sufficiently small,

- $F \leq K$ and $F(p)=c=K(p)$ is the absolute maximum of $F$,

- $F^{s}:=G^{s} \# F \leq H_{+}$for all $s$.

Note that here we do not require $F$ to be supported in $V$, but only to be constant and equal to $\min F$ outside $V$. By Example $2.5, F^{s}$ is an isospectral homotopy beginning with

$$
H_{-}:=G^{0} \# F \leq G^{0} \# K=H
$$

and ending with $F^{1}=F$. Throughout this homotopy, $F^{s} \leq H_{+}$.

Furthermore, the homotopy $\left(F^{s}\right)^{(k)}$ from $H_{-}^{(k)}$ to $F^{(k)}$ is isospectral and $\left(F^{s}\right)^{(k)} \leq$ $H_{+}^{(k)}$ for all $s$. (Note in this connection that in general an iteration of an isospectral homotopy need not be isospectral. However, an iteration of a homotopy of the form $G^{s} \# K$, where all $G^{s}$ generate loops of Hamiltonian diffeomorphisms and are normalized, is automatically isospectral.)

\subsubsection{The Floer homology of $H_{ \pm}$and the monotone homotopy map When the} manifold $M$ is symplectically aspherical, the Floer homology groups of the functions $H_{+}^{(k)} \geq F^{(k)}$, for the intervals in question, and the monotone homotopy map are determined in [20]. In fact, it suffices to carry out the calculation for $M=\mathbb{R}^{2 n}$, which is done in [26], for then, by Example 5.6, the result holds for any symplectically aspherical manifold whenever $\epsilon$ is small. When $M=\mathbb{R}^{2 n}$ and $\epsilon<\epsilon\left(U, W, \mathbb{R}^{2 n}\right)$, it is shown that the map

$$
\mathbb{F} \cong \mathrm{HF}_{2 n+1}^{\left(k c+\delta_{k}, k c+\epsilon\right)}\left(H_{+}^{(k)}\right) \stackrel{\cong}{\rightarrow} \mathrm{HF}_{2 n+1}^{\left(k c+\delta_{k}, k c+\epsilon\right)}\left(F^{(k)}\right) \cong \mathbb{F}
$$

is an isomorphism. (At this point we use the requirement that $k\left\|d^{2} F_{p}\right\|_{\xi_{p}}$ is sufficiently small.)

Thus, returning to the setting of Theorem 1.17, we have the isomorphism

$$
\mathbb{F} \cong \mathrm{HF}_{2 n+1}^{\left(k c+\delta_{k}, k c+\epsilon\right)}\left(H_{+}^{(k)} ; U\right) \stackrel{\cong}{\rightrightarrows} \mathrm{HF}_{2 n+1}^{\left(k c+\delta_{k}, k c+\epsilon\right)}\left(F^{(k)} ; U\right) \cong \mathbb{F},
$$

by (5-6) and Proposition 5.4. Since $\epsilon<\epsilon(U, W, M)$ and hence these groups enter the filtered Floer homology of $H_{+}^{(k)}$ and $F^{(k)}$ as direct summands, we conclude that the map

$$
\mathrm{HF}_{2 n+1}^{\left(k c+\delta_{k}, k c+\epsilon\right)}\left(H_{+}^{(k)}\right) \stackrel{\neq 0}{\longrightarrow} \mathrm{HF}_{2 n+1}^{\left(k c+\delta_{k}, k c+\epsilon\right)}\left(F^{(k)}\right)
$$

is nonzero. 
Consider the diagram

$$
\begin{aligned}
& \mathrm{HF}_{2 n+1}^{\left(k c+\epsilon, k c+\delta_{k}\right)}\left(H_{+}^{(k)}\right)
\end{aligned}
$$

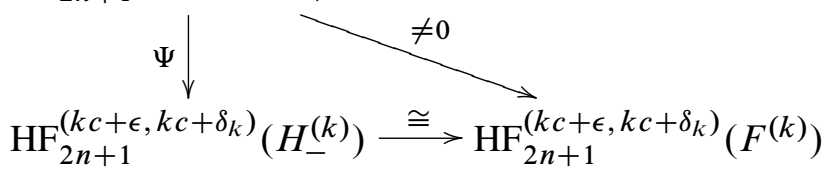

where the horizontal isomorphism is induced by the isospectral homotopy $\left(F^{s}\right)^{(k)}$ from $H_{-}^{(k)}$ to $F^{(k)}$ and the remaining two arrows are monotone homotopy maps. Since $\left(F^{s}\right)^{(k)} \leq H_{+}^{(k)}$ for all $s$, the diagram commutes; see Section 2.2.2 and, in particular, Example 2.5 and the diagram (2-5). As we have just shown, the diagonal arrow is a nonzero map, and hence so is the map $\Psi$. This concludes the proof of the theorem.

Remark 5.10 Note that this argument, reducing the calculation to the case of $M=$ $\mathbb{R}^{2 n}$, also provides a simple ending of the original proof from [20].

\section{The Ljusternik-Schnirelman theory}

Our goal of this section is to prove Theorems 1.7 and 1.12. The proofs differs significantly from the proofs of other theorems in this paper and rely on the machinery of action selectors and the Hamiltonian Ljusternik-Schnirelman theory.

\subsection{The classical Ljusternik-Schnirelman theory: critical value selectors}

To set the stage for an overview of the theory of Hamiltonian action selectors and for the proof of Theorem 1.7, let us briefly recall the definition and basic properties of classical critical value selectors in the Ljusternik-Schnirelman theory.

Let $M$ be a closed, $m$-dimensional manifold and $f \in C^{\infty}(M)$. To $u \in H_{*}(M)$, we associate the critical value selector by the formula

$$
\mathrm{c}_{u}^{\mathrm{LS}}(f)=\inf \left\{a \mid u \in \operatorname{im}\left(i^{a}\right)\right\}=\inf \left\{a \mid j^{a}(u)=0\right\},
$$

where $i^{a}: H_{*}(\{f \leq a\}) \rightarrow H_{*}(M)$ and $j^{a}: H_{*}(M) \rightarrow H_{*}(M,\{f \leq a\})$ are the natural "inclusion" and "quotient" maps. By definition, $\mathrm{c}_{0}^{\mathrm{LS}}(f)=-\infty$. As is well known, $\mathrm{c}_{u}^{\mathrm{LS}}(f)$ has the following properties:

- $\quad \mathrm{c}_{u}^{\mathrm{LS}}($ const $)=$ const $($ normalization $)$ and

$$
\mathrm{c}_{[\mathrm{pt}]}^{\mathrm{LS}}(f)=\min f \leq \mathrm{c}_{u}^{\mathrm{LS}}(f) \leq \max f=\mathrm{c}_{[M]}^{\mathrm{LS}}(f) .
$$


- $\mathrm{c}_{u}^{\mathrm{LS}}(f)$ is Lipschitz in $f$ with respect to the $C^{0}$-topology (continuity).

- $\mathrm{c}_{u \cap w}^{\mathrm{LS}}(f+g) \leq \mathrm{c}_{u}^{\mathrm{LS}}(f)+\mathrm{c}_{w}^{\mathrm{LS}}(g)$ (the triangle inequality) and $\mathrm{c}_{\alpha u}^{\mathrm{LS}}(f)=\mathrm{c}_{u}^{\mathrm{LS}}(f)$ for any nonzero $\alpha \in \mathbb{F}$.

- $\mathrm{c}_{u}^{\mathrm{LS}}(f)$ is a critical value of $f$ (criticality or the minimax principle).

- $\mathrm{c}_{u \cap w}^{\mathrm{LS}}(f) \leq \mathrm{c}_{u}^{\mathrm{LS}}(f)$ and, moreover, if $w \neq[M]$ and the critical points of $f$ are isolated, we have strict inequality

$$
\mathrm{c}_{u \cap w}^{\mathrm{LS}}(f)<\mathrm{c}_{u}^{\mathrm{LS}}(f) .
$$

The proofs of these well-known facts can be easily extracted from any standard treatment of the Ljusternik-Schnirelman theory.

As an immediate consequence of (6-1), we obtain the classical result of Ljusternik and Schnirelman asserting that the number of distinct critical values of $f$ is no smaller than $\operatorname{cl}(M)+1$, whenever the critical points of $f$ are isolated; see, eg, Hofer and Zehnder [34] and references therein. Here, $\operatorname{cl}(M)$ stands for the cup-length of $M$ over $\mathbb{F}$, ie, the maximal number $k$ of elements $u_{1}, \ldots, u_{k}$ in $H_{*<m}(M)$ such that $u_{1} \cap \cdots \cap u_{k} \neq 0$. For this reason, we will refer to (6-1) as the Ljusternik-Schnirelman inequality.

Remark 6.1 The Ljusternik-Schnirelman inequality (6-1) can be refined as follows. Assume that $\mathrm{c}_{u \cap w}^{\mathrm{LS}}(f)=\mathrm{c}_{u}^{\mathrm{LS}}(f)$ and let $\Sigma$ be the set of critical points of $f$ on the level $\left\{f=\mathrm{c}_{u}^{\mathrm{LS}}(f)\right\}$. Denote by $w^{*} \in H^{*}(M)$ the Poincare dual of $w$. Then the restriction of $w^{*}$ to any neighborhood of $\Sigma$ is nonzero. In particular, the image of $w^{*}$ in the Alexander-Spanier cohomology of $\Sigma$ is also nonzero. When $w=[M]$ this implies that $\Sigma \neq \varnothing$ (criticality). If $w \in H_{*<m}(M)$, we infer that for the strict inequality (6-1) to fail, the critical set $\Sigma$ must contain a cycle of degree $m-\operatorname{deg} w>0$ comprised of critical points, and hence $\Sigma$ must be infinite.

\subsection{The Hamiltonian Ljusternik-Schnirelman theory: action selectors}

The theory of Hamiltonian action selectors or spectral invariants, as they are usually referred to, can be viewed as the theory of critical value selectors applied to the action functional $\mathcal{A}_{H}$ in place of a smooth function $f$. A complete Floer-theoretic version of such a theory for symplectically aspherical manifolds was developed by Schwarz [52] although the first versions of the theory go back to Hofer and Zehnder [34] and Viterbo [56]. (Applications of action selectors extend far beyond the scope of our discussion here; see, eg, Frauenfelder and Schlenk [19], Entov and Polterovich [10; 9], Ginzburg [21], Gürel [29], Hofer and Zehnder [34], McDuff and Salamon [42], 
Schwarz [52] and Viterbo [56] and references therein.) In this section, we mainly follow Oh [44], where the action selectors are studied for Hamiltonians on closed, weakly monotone manifolds.

6.2.1 Basic properties of the action selectors Let $M$ be such a manifold and let $H$ be a Hamiltonian on $M$. To a nonzero element $u \in \mathrm{HQ}_{*}(M) \cong \mathrm{HF}_{*}(H)$, we associate the action selector $\mathrm{c}_{u}$ by a formula virtually identical to the definition of $\mathrm{c}^{\mathrm{LS}}$ :

$$
\mathrm{c}_{u}(H)=\inf \left\{a \in \mathbb{R} \backslash \mathcal{S}(H) \mid u \in \operatorname{im}\left(i^{a}\right)\right\}=\inf \left\{a \in \mathbb{R} \backslash \mathcal{S}(H) \mid j^{a}(u)=0\right\},
$$

where now $i^{a}: \mathrm{HF}_{*}^{(-\infty, a)}(H) \rightarrow \mathrm{HF}_{*}(H)$ and $j^{a}: \mathrm{HF}_{*}(H) \rightarrow \mathrm{HF}_{*}^{(a, \infty)}(H)$ are the natural "inclusion" and "quotient" maps; see Section 2.2.1. Then $\mathrm{c}_{u}(H)>-\infty$. (This is a nontrivial statement unless $M$ is assumed to be rational; see Oh [44] for a proof.) As in the finite-dimensional case, by definition, $\mathrm{c}_{0}(H)=-\infty$. In what follows, for the sake of convenience, we will always assume that $u$ is homogeneous. (This assumption is not really necessary: $u$ could be a sum of homology classes of different degrees.) When $H$ is nondegenerate,

$$
\mathrm{c}_{u}(H)=\inf _{[\sigma]=u} \mathcal{A}_{H}(\sigma),
$$

where we set $\mathcal{A}_{H}(\sigma)=\max \left\{\mathcal{A}_{H}(\bar{x}) \mid \alpha_{\bar{x}} \neq 0\right\}$ for $\sigma=\sum \alpha_{\bar{x}} \bar{x} \in \mathrm{CF}_{*}(H)$.

The action selectors $\mathrm{c}_{u}$ have the following properties:

(AS1) Normalization: $\mathrm{c}_{u}(H)=\mathrm{c}_{u}^{\mathrm{LS}}(H)$ if $u \in H_{*}(M)$ and $H$ is autonomous and $C^{2}$-small.

(AS2) Continuity: $\mathrm{c}_{u}$ is Lipschitz in $H$ in the $C^{0}$-topology.

(AS3) Monotonicity: $\mathrm{c}_{u}(H) \geq \mathrm{c}_{u}(K)$, whenever $H \geq K$ pointwise.

(AS4) Hamiltonian shift: $\mathrm{c}_{u}(H+a(t))=\mathrm{c}_{u}(H)+\int_{0}^{1} a(t) d t$, where $a: S^{1} \rightarrow \mathbb{R}$.

(AS5) Symplectic invariance: $\mathrm{c}_{\varphi_{*}(u)}(H)=\mathrm{c}_{u}\left(\varphi^{*} H\right)$ for any symplectomorphism $\varphi$, and hence $\mathrm{c}_{u}(H)=\mathrm{c}_{u}\left(\varphi^{*} H\right)$ if $\varphi$ is isotopic to id in the group of symplectomorphisms.

(AS6) Homotopy invariance: $\mathrm{c}_{u}(H)=\mathrm{c}_{u}(K)$, when $\varphi_{H}=\varphi_{K}$ in the universal covering of the group of Hamiltonian diffeomorphisms, both $H$ and $K$ are normalized, and $M$ is rational.

(AS7) Quantum shift: $\mathrm{c}_{\alpha u}(H)=\mathrm{c}_{u}(H)+I_{\omega}(\alpha)$, where $\alpha \in \Lambda$.

(AS8) Valuation inequality: $\mathrm{c}_{u+w}(H) \leq \max \left\{\mathrm{c}_{u}(H), \mathrm{c}_{w}(H)\right\}$ and, moreover, the inequality is strict if $\mathrm{c}_{u}(H) \neq \mathrm{c}_{w}(H)$.

(AS9) Triangle inequality: $\mathrm{c}_{u * w}(H \# K) \leq \mathrm{c}_{u}(H)+\mathrm{c}_{w}(K)$. 
(AS10) Spectrality: $\mathrm{c}_{u}(H) \in \mathcal{S}(H)$, provided that $M$ is rational or otherwise $H$ is nondegenerate. More specifically, there exists a capped one-periodic orbit $\bar{x}$ of $H$ such that $\mathrm{c}_{u}(H)=\mathcal{A}_{H}(\bar{x})$ and $0 \leq \operatorname{deg} u-\Delta_{H}(\bar{x}) \leq 2 n$. Furthermore, $\operatorname{HF}_{\operatorname{deg} u}(H, \bar{x}) \neq 0$ if $x$ is isolated. In particular, $\mu_{\mathrm{CZ}}(\bar{x})+n=\operatorname{deg} u$ when $H$ is nondegenerate.

We refer the reader to [44] for the proofs of (AS1)-(AS9); see also Entov [8], Entov and Polterovich [9], Schwarz [52] and McDuff and Salamon [42]. Some of these properties (eg, (AS7) and (AS8)) follow immediately from the definitions, while some (eg, (AS9)) are quite nontrivial to establish. The spectrality property (AS10) is proved in [44] in the rational case and in [54] for nondegenerate Hamiltonians.

6.2.2 The Hamiltonian Ljusternik-Schnirelman inequality The additional property of the action selector that is needed for the proof of Theorem 1.7 is an analogue of the Ljusternik-Schnirelman inequality (6-1). Set

$$
\mathrm{HQ}_{*}^{-}(M)=H_{*<2 n}(M) \otimes \Lambda .
$$

Thus, $\mathrm{HQ}_{*}(M)=\mathrm{HQ}_{*}^{-}(M) \oplus[M] \Lambda$, but, in general, $\mathrm{HQ}_{*}^{-}(M)$ is not a subalgebra of $\mathrm{HQ}_{*}(M)$.

Proposition 6.2 (Hamiltonian Ljusternik-Schnirelman inequality) For any $H$ and $u$ and $w$ in $\mathrm{HQ}_{*}(M)$ we have $\mathrm{c}_{u * w}(H) \leq \mathrm{c}_{u}(H)+I_{\omega}(w)$. Moreover,

$$
\mathrm{c}_{u * w}(H)<\mathrm{c}_{u}(H)+I_{\omega}(w),
$$

provided that $w \in \mathrm{HQ}_{*}^{-}(M)$ and the periodic orbits of $H$ are isolated.

The authors are not aware of any reference to a proof of this proposition although the result bears a similarity to arguments from, eg, $[14 ; 31 ; 37 ; 51]$. (For instance, for rational manifolds, the result appears to be implicitly contained in [51] and the symplectically aspherical case is treated in [52].) Since Proposition 6.2 is particularly important for what follows, we outline its proof, which is reminiscent of the arguments in [29].

Proof Observe that $\mathrm{c}_{u}(0)=I_{\omega}(u)$ as is clear from (AS1) and (AS7). Then the nonstrict inequality follows immediately from the triangle inequality (AS9) with $K \equiv 0$. The proof of the strict inequality (6-2) is based on several preliminary observations. Consider first an arbitrary class $w=\sum_{A} w_{A} e^{A} \in \mathrm{HQ}_{*}(M)$. We claim that

$$
\mathrm{c}_{w}(g) \leq \sup _{A}\left\{\mathrm{c}_{w_{A}}^{\mathrm{LS}}(g)\right\}+I_{\omega}(w)
$$


when $g$ is a sufficiently $C^{2}$-small function on $M$. (Here we omitted the requirement $w_{A} \neq 0$ since $\mathrm{c}_{0}^{\mathrm{LS}}(g)=-\infty$.) To prove (6-3), note that, by (AS1) and (AS8),

$$
\begin{aligned}
\mathrm{c}_{w}(g) & \leq \sup _{A, w_{A} \neq 0}\left\{\mathrm{c}_{w_{A}}^{\mathrm{LS}}(g)+I_{\omega}(A)\right\} \\
& \leq \sup _{A, w_{A} \neq 0}\left\{\mathrm{c}_{w_{A}}^{\mathrm{LS}}(g)\right\}+\sup _{A, w_{A} \neq 0}\left\{I_{\omega}(A)\right\} \\
& =\sup _{A}\left\{\mathrm{c}_{w_{A}}^{\mathrm{LS}}(g)\right\}+I_{\omega}(w) .
\end{aligned}
$$

Fix now a finite collection of points $p_{1}, \ldots, p_{m}$ in $M$ and assume that $w \in \mathrm{HQ}_{*}^{-}(M)$. Let $g \leq 0$ be a $C^{2}$-small function on $M$ which is equal to zero on small neighborhoods of these points and otherwise strictly negative. Then, clearly, $\mathrm{c}_{v}^{\mathrm{LS}}(g)<-\delta_{g}<0$ for all $v \in H_{*<2 n}(M)$ and some $\delta_{g}>0$ depending of $g$. Thus, by (6-3), we have

$$
\mathrm{c}_{w}(g) \leq I_{\omega}(w)-\delta_{g}<I_{\omega}(w) .
$$

Furthermore, let $a: S^{1} \rightarrow \mathbb{R}$ be a nonnegative, $C^{2}$-small function, equal to zero outside a small neighborhood of $0 \in S^{1}$. Set $f=a g$. The Hamiltonian flow of $f$ is a reparametrization of the flow of $g$ through time $\epsilon=\int_{0}^{1} a(t) d t$. Hence, when $g$ and $a$ are sufficiently $C^{2}$-small, $\epsilon g$ and $f$ have the same periodic orbits (the critical points of $g$ ) and the same action spectrum. Clearly, this is also true for every function in the linear family $f_{s}=(1-s) \epsilon g+s f$, with $s \in[0,1]$, connecting $\epsilon g$ and $f$. By the continuity of $\mathrm{c}_{w}$ (see (AS2)), we conclude that $\mathrm{c}_{w}(\epsilon g)=\mathrm{c}_{w}(f)$ and (6-4) turns into

$$
\mathrm{c}_{w}(f) \leq I_{\omega}(w)-\delta_{\epsilon g}<I_{\omega}(w) .
$$

Now we are in a position to finish the proof. Let $p_{1}, \ldots, p_{m}$ be the fixed points of $\varphi_{H}$. Consider the Hamiltonian $H \# f$. Assume that the neighborhoods of the fixed points on which $g \equiv 0$ are sufficiently small, the interval on which $a>0$ is sufficiently short, and also $g$ and $a$ are $C^{2}$-small. Then it is not hard to see that $H \# f$ has the same periodic orbits as $H$ and, moreover, $\mathcal{S}(H \# f)=\mathcal{S}(H)$. As above, the same holds for all Hamiltonians $H \#(s f)$ with $s \in[0,1]$. Again, by the continuity property (AS2), we have $\mathrm{c}_{u * w}(H)=\mathrm{c}_{u * w}(H \# f)$. Finally, using the triangle inequality (AS9) and (6-5), we obtain

$$
\mathrm{c}_{u * w}(H)=\mathrm{c}_{u * w}(H \# f) \leq \mathrm{c}_{u}(H)+\mathrm{c}_{w}(f)<\mathrm{c}_{u}(H)+I_{\omega}(w) .
$$

6.2.3 Digression: The Arnold conjecture Proposition 6.2 provides a natural framework for a discussion of the degenerate case of the Arnold conjecture asserting that the number of one-periodic orbits of $H$ is bounded from below by $\operatorname{cl}(M)+1$. (The conjecture is still open for a general rational, weakly monotone manifold $M$.) For instance, the proposition immediately implies the degenerate case of the conjecture 
when $M$ is symplectically aspherical, originally proved by Floer [13] and Hofer [31]; see also Hofer and Zehnder [34]. Although in the presence of nontrivial cappings Proposition 6.2 does not address one of the main difficulties - showing that the orbits found are geometrically distinct - it allows one to easily reprove some of the known results. Here, for the sake of completeness, we illustrate this utilization of Proposition 6.2 by several examples.

The general line of reasoning is absolutely standard. Namely, assume that we have $m+1$ nonzero homology classes $[M]=u_{0}, u_{1}, \ldots, u_{m}$ in $\mathrm{HQ}_{*}(M)$ such that $u_{k}=$ $u_{k-1} * w_{k}$ with $w_{k} \in \mathrm{HQ}_{*}^{-}(M)$ and $I_{\omega}\left(w_{k}\right) \leq 0$. Then, by Proposition 6.2, $\mathrm{c}_{u_{k}}(H)<$ $\mathrm{c}_{u_{k-1}}(H)$ for any Hamiltonian $H$ on $M$ with isolated fixed points. (Every weakly monotone $M$ admits $\mathrm{cl}(M)+1$ such classes.) Thus, when the spectrality property (AS10) holds, we have $m+1$ capped one-periodic orbits $\bar{x}_{0}, \ldots, \bar{x}_{m}$ such that

$$
\mathcal{A}_{H}\left(\bar{x}_{k}\right)<\mathcal{A}_{H}\left(\bar{x}_{k-1}\right) \text { and }\left|\Delta_{H}\left(\bar{x}_{k}\right)-\operatorname{deg} u_{k}\right| \leq 2 n .
$$

In general, the orbits $x_{k}$ need not be distinct, and showing that at least some of the orbits are requires additional assumptions on $M$ and presents the main difficulty in proving the Arnold conjecture even when $M$ is rational and weakly monotone. Below, obtaining a lower bound on the number of geometrically distinct one-periodic orbits, we closely follow the original sources, with some simplifications afforded by Proposition 6.2 and the usage of the mean index and restrict our attention only to the examples where this lower bound is nearly obvious.

Among the results that readily follow from Proposition 6.2 are, for instance:

(i) The Arnold conjecture for rational manifolds, when $\|H\|<\lambda_{0}[51]$.

(ii) The Arnold conjecture for negatively monotone manifolds with $N \geq n$ [37].

(iii) The Arnold conjecture for $\mathbb{C P}^{n}[16 ; 17 ; 14]$ or, more generally, the existence of at least $n+1$ distinct orbits when $M=\mathbb{C} \mathbb{P}^{n} \times P$, where $P$ is symplectically aspherical [43; 51], and the existence of $\operatorname{gcd}\left(n_{1}+1, \ldots, n_{l}+1\right)$ distinct orbits on a monotone product $M=\mathbb{C} \mathbb{P}^{n_{1}} \times \cdots \times \mathbb{C P}^{n_{l}}[14]$.

(iv) The existence of at least $2 N /(2 n-d)$ distinct one-periodic orbits when $M$ is monotone with $N>0$ and there is a nonnilpotent element $w \in \mathrm{HQ}_{d<2 n}(M)$ [51].

A brief note on the proofs In all four cases, the choice of the elements $u_{k}$ is the most straightforward. For instance, in (i) and in (ii), we can take $w_{k} \in H_{*<2 n}(M)$ such that $w_{1} \cap \cdots \cap w_{m}=1$ and $m=\operatorname{cl}(M)$. Then, in (i), the assertion that the orbits $x_{k}$ are geometrically distinct follows immediately from the well-known bound $0 \leq \mathrm{c}_{[M]}(H)-\mathrm{c}_{1}(H) \leq\|H\|$; see, eg, Oh [44] and Schwarz [52]. In (ii), this is a consequence of the assumption that $M$ is negative monotone and $N \geq n$ and of the 
bound $\Delta_{H}\left(\bar{x}_{j}\right)-\Delta_{H}\left(\bar{x}_{i}\right) \geq d_{i}-d_{j}-2 n>-2 n$, where $j>i$ and $d_{k}=\operatorname{deg} u_{k}$, which, in turn, follows from the second part of (6-6).

In (iii), with $M=\mathbb{C} \mathbb{P}^{n} \times P$, we have $u_{k}=w^{k} \otimes[P]$, where $w$ is the generator of $H_{2 n-2}\left(\mathbb{C} \mathbb{P}^{n}\right)$, and $m=n$; cf Example 2.7. The fact that all orbits are geometrically distinct can be easily seen from, eg, Lemma 6.6. The case of the monotone product of projective spaces is treated in a similar way, keeping track of both the action and the mean index. Alternatively, (iii) follows immediately from (iv).

In (iv), the argument is slightly different $[16 ; 51]$. Set $u_{k}=w^{k}$ with $k=0,1, \ldots$ and consider the infinite collection of the orbits $\bar{x}_{k}$. Note that deg $u_{k}=d k-2 n(k-1)$, when $k \geq 1$. Thus, by (6-6), this collection contains $L /(2 n-d)$ orbits with mean index in the range $[-L, 0]$, up to an error independent of $L \gg 0$. Assume that there are exactly $l$ geometrically distinct orbits within the collection $\left\{\bar{x}_{k}\right\}$. Each of these orbits contributes at most $L / 2 N$ capped orbits to the list of orbits with mean index in $[-L, 0]$, again up to a bounded error. Thus, we can totally have at most $l L / 2 N$ orbits with action in $[-L, 0]$. Dividing by $L \rightarrow \infty$, we conclude that $l \geq 2 N /(2 n-d)$. $\square$

Remark 6.3 All of the above arguments concern, in fact, the action or/and mean index spectrum of $H$. For instance, in (i) and in (iii) with $M=\mathbb{C} \mathbb{P}^{n} \times P$, we showed that $\mathcal{S}(H)$ contains at least $\operatorname{cl}(M)+1$ distinct points in the interval $\left[\mathrm{c}_{1}(H), \mathrm{c}_{[M]}(H)\right]$ of length not exceeding $\lambda_{0}$; cf Theorem 1.7 and Theorem 6.4. The proof of (ii) is based on a lower bound on the mean index gap $\Delta_{H}\left(\bar{x}_{j}\right)-\Delta_{H}\left(\bar{x}_{i}\right)$. The assertion (iv) relies on an estimate of the asymptotic density of the mean index spectrum. Namely, we showed that the asymptotic density (defined in the obvious way) is bounded from below by $1 /(2 n-d)$. On the other hand, every periodic orbit generates, via recapping, a set of orbits with density $1 / 2 N$. Hence, the number of distinct orbits is at least $2 N /(2 n-d)$.

\subsection{The a priori bound on the action-index gap}

The goal of this section is to prove Theorem 1.7. We establish the following slightly more general result:

Theorem 6.4 Let $\left(M^{2 n}, \omega\right)$ be a closed, weakly monotone symplectic manifold such that there exist $u \in \mathrm{HQ}_{*}^{-}(M)$ and $w \in \mathrm{HQ}_{*}^{-}(M)$ and an element $\alpha \in \Lambda$ satisfying the homological condition of Theorem 1.7:

$$
[M]=(\alpha u) * w .
$$

Let $H$ be a Hamiltonian on $M$ with isolated one-periodic orbits. Assume in addition that $I_{\omega}(u) \leq 0$ and one of the following conditions holds: 
(a) $\quad M$ is rational and $I_{\omega}(\alpha)+I_{\omega}(w) \leq \lambda_{0}$.

(b) $0<|2 n-\operatorname{deg} u|<2 N$ and $H$ is nondegenerate.

Then the flow of $H$ has two geometrically distinct (capped) one-periodic orbits $\bar{x}$ and $\bar{y}$ such that

$$
\begin{aligned}
& 0 \leq-I_{\omega}(u)<\mathcal{A}_{H}(\bar{x})-\mathcal{A}_{H}(\bar{y})<I_{\omega}(\alpha)+I_{\omega}(w), \\
& \left|\Delta_{H}(\bar{x})-n\right| \leq n \quad \text { and } \quad 0 \leq \operatorname{deg} u-\Delta_{H}(\bar{y}) \leq 2 n .
\end{aligned}
$$

Remark 6.5 When the Hamiltonian $H$ is nondegenerate, the mean index bounds (6-8) can be replaced by

$$
\mu_{\mathrm{CZ}}(\bar{x})=n \quad \text { and } \quad \mu_{\mathrm{CZ}}(\bar{y})+n=\operatorname{deg} u .
$$

Furthermore, if $H$ is weakly nondegenerate, all inequalities in (6-8) are strict.

To obtain Theorem 1.7 as a consequence of Theorem 6.4, it suffices to notice that $\left.I_{\omega}\right|_{H_{*}(M)}=0$.

Proof of Theorem 6.4 The first step in the argument is the following simple observation:

Lemma 6.6 Assume that quantum homology classes $u \in \mathrm{HQ}_{*}^{-}(M)$ and $w \in \mathrm{HQ}_{*}^{-}(M)$ and $\alpha \in \Lambda$ meet the homological condition of Theorem 1.7 and Theorem 6.4. Let $H$ be a Hamiltonian on $M$ with isolated one-periodic orbits. Then

$$
-I_{\omega}(u)<\mathrm{c}_{[M]}(H)-\mathrm{c}_{u}(H)<I_{\omega}(\alpha)+I_{\omega}(w) .
$$

Proof Since $u=u *[M]$, where $u \in \mathrm{HQ}_{*}^{-}(M)$, and since the periodic orbits of $H$ are isolated, we have, by Proposition 6.2,

$$
\mathrm{c}_{u}(H)<\mathrm{c}_{[M]}(H)+I_{\omega}(u),
$$

which implies the first inequality in (6-10). Likewise, using again Proposition 6.2, we infer from the homological condition that

$$
\mathrm{c}_{[M]}(H)<\mathrm{c}_{\alpha u}(H)+I_{\omega}(w)=\mathrm{c}_{u}(H)+I_{\omega}(\alpha)+I_{\omega}(w) .
$$

Hence, the second inequality in (6-10) also holds. 
Returning to the proof of the theorem, recall that the spectrality condition, (AS10), holds due to the assumption that $M$ is rational or, otherwise, $H$ is nondegenerate. Hence, there exist capped one-periodic orbits $\bar{x}$ and $\bar{y}$ satisfying (6-8) and such that

$$
\mathcal{A}_{H}(\bar{x})=\mathrm{c}_{[M]}(H) \text { and } \mathcal{A}_{H}(\bar{y})=\mathrm{c}_{u}(H) .
$$

Moreover, if $H$ is nondegenerate, we have (6-9). Now the action bound (6-7) follows from (6-10) and the condition that $I_{\omega}(u) \leq 0$.

It remains to show that $x$ and $y$ are geometrically distinct, ie, $\bar{x} \neq \bar{y}$ and $\bar{y}$ is not a nontrivial recapping of $\bar{x}$. The fact that $\bar{x} \neq \bar{y}$ follows immediately from the first inequality in (6-7): $\mathcal{A}_{H}(\bar{x})-\mathcal{A}_{H}(\bar{y})>0$.

Moreover, if $\bar{x}=\bar{y} \# A$, we necessarily have $I_{\omega}(A)>0$. Thus $\mathcal{A}_{H}(\bar{x})-\mathcal{A}_{H}(\bar{y})=\lambda_{0} k$, where $k$ is a positive integer, when $M$ is rational. In case (a), this is impossible since $\mathcal{A}_{H}(\bar{x})-\mathcal{A}_{H}(\bar{y})<\lambda_{0}$ by the second inequality of (6-7).

Next, let us consider case (b), when $H$ is nondegenerate but $M$ is not necessarily rational. Then (6-9) holds, and

$$
0<\left|\mu_{\mathrm{CZ}}(\bar{x})-\mu_{\mathrm{CZ}}(\bar{y})\right|<2 N .
$$

Thus, if $\bar{x}=\bar{y} \# A$, we have $0<\left|I_{c_{1}}(A)\right|=\left|\mu_{\mathrm{CZ}}(\bar{x})-\mu_{\mathrm{CZ}}(\bar{y})\right|<2 N$, which is impossible.

Remark 6.7 Note that in the last argument the nondegeneracy assumption on $H$ is used twice: the first time to ensure the spectrality condition and the second time to prove, using (6-11), that $\bar{x}$ is not a recapping of $\bar{y}$. Hence, even if spectrality for degenerate Hamiltonian were established, the proof would still rely on the nondegeneracy assumption.

Remark 6.8 As is easy to see, Theorem 6.4 can be further generalized as follows. Let $M$ be closed and weakly monotone. Assume that there exists $v \in \mathrm{HQ}_{*}(M)$ and three classes $u, w$ and $w^{\prime}$ in $\mathrm{HQ}_{*}^{-}(M)$, and also $\alpha \in \Lambda$, such that

$$
u=v * w^{\prime} \quad \text { and } \quad v=(\alpha u) * w
$$

and $I_{\omega}\left(w^{\prime}\right) \leq 0$. Let the Hamiltonian $H$ have isolated one-periodic orbits. Assume furthermore that $M$ is rational and $I_{\omega}(\alpha)+I_{\omega}(w)<\lambda_{0}$ or $0<|\operatorname{deg} v-\operatorname{deg} u|<2 N$ and $H$ is nondegenerate. Then $H$ has two geometrically distinct (capped) one-periodic orbits $\bar{x}$ and $\bar{y}$ such that the inequality (6-7) holds with $u$ replaced by $w^{\prime}$, and (6-8) holds with the first bound replaced by $\left|\Delta_{H}(\bar{x})-\operatorname{deg} v\right| \leq 2 n$. When $H$ is nondegenerate, we have $\mu_{\mathrm{CZ}}(\bar{x})+n=\operatorname{deg} v$ and $\mu_{\mathrm{CZ}}(\bar{y})+n=\operatorname{deg} u$; cf Remark 6.5. Setting $v=[M]$ and $w^{\prime}=u$, we obtain Theorem 6.4. 


\subsection{Proof of Theorem 1.12}

By Example 2.7, $\mathrm{HQ}_{*}\left(\mathbb{C} \mathbb{P}^{n}\right)=\mathbb{F}$, when the degree $*$ is even and zero otherwise. Let $u_{n-1}$ be a generator of $\mathrm{HQ}_{2 n-2}\left(\mathbb{C} \mathbb{P}^{n}\right)$. Then $u_{n-i}:=u_{n-1}^{i}$ is a generator of $\mathrm{HQ}_{2(n-i)}\left(\mathbb{C P}^{n}\right)$. In particular, $u_{n}=[M]$ and $u_{0}=[\mathrm{pt}]$ and $u_{-1}=q^{-1}[M]$, etc., where $q=e^{A}$ with $I_{\omega}(A)=\lambda_{0}$ and $I_{c_{1}}(A)=2(n+1)$.

Consider first a Hamiltonian $H$ on $\mathbb{C P}^{n}$ with exactly $n+1$ distinct one-periodic orbits. Then, by Proposition 6.2, there is a one-to-one correspondence between its capped one-periodic orbits $\bar{x}$ and the generators $u_{j}$ given by

$$
\mathcal{A}_{H}(\bar{x})=\mathrm{c}_{u_{j}}(H) \text {. }
$$

Set $\mu_{H}(\bar{x}):=2 j=\operatorname{deg} u_{j}$. For instance, if $\bar{x}$ is nondegenerate, then $\mu_{H}(\bar{x})=$ $\mu_{\mathrm{CZ}}(H, \bar{x})+n$. Thus, the collection of capped orbits is strictly ordered by the index $\mu_{H}$. Furthermore, as readily follows from Proposition 6.2 and Example 2.7, this collection is also strictly ordered by the action and these two orderings coincide:

$$
\mu_{H}(\bar{x})>\mu_{H}(\bar{y}) \text { if and only if } \quad \mathcal{A}_{H}(\bar{x})>\mathcal{A}_{H}(\bar{y}) .
$$

This observation is in fact the key point of the proof. Note also that, again by (AS10), we have

$$
0 \leq \mu_{H}(\bar{x})-\Delta_{H}(\bar{x}) \leq 2 n .
$$

Assume now that $H$ is as in the statement of the theorem: for every $k$, the flow of $H$ has exactly $n+1$ distinct $k$-periodic orbits. (Then every such periodic orbit is necessarily the $k$-th iteration of a one-periodic orbit of $H$.) The above considerations apply to $H^{(k)}$ for all $k>0$. Recall also that

$$
\mathcal{A}_{H^{(k)}}\left(\bar{x}^{k}\right)=k \mathcal{A}_{H}(\bar{x}) \quad \text { and } \quad \Delta_{H^{(k)}}\left(\bar{x}^{k}\right)=k \Delta_{H}(\bar{x})
$$

by, eg, (MI). Hence, as follows from (6-13),

$$
\lim _{k \rightarrow \infty} \frac{\mu_{H^{(k)}}\left(\bar{x}^{k}\right)}{k}=\Delta_{H}(\bar{x}) .
$$

Fix two capped one-periodic orbits $\bar{x}$ and $\bar{y}$ of $H$ with, say,

$$
\mathcal{A}_{H}(\bar{x})>\mathcal{A}_{H}(\bar{y}) \quad \text { and } \quad \mu(\bar{x})>\mu(\bar{y}) .
$$

Our goal is to show that $\tilde{\mathcal{A}}_{H}(x)=\tilde{\mathcal{A}}_{H}(y)$, where $\tilde{\mathcal{A}}_{H}=\mathcal{A}_{H}-\lambda \Delta_{H}$. Without loss of generality, we may assume that $\mathcal{A}_{H}(\bar{y})>0$.

Let $l$ be a positive integer such that

$$
\mathcal{A}_{H}(\bar{y} \#(l A))=\mathcal{A}_{H}(\bar{y})+l \lambda_{0}>\mathcal{A}_{H}(\bar{x}) .
$$


The sequence $\mathcal{A}_{H^{(k)}}\left(\bar{y}^{k} \#(l A)\right)=k \mathcal{A}_{H}(\bar{y})+l \lambda_{0}$ grows slower, as a function of $k$, than the sequence $\mathcal{A}_{H^{(k)}}\left(\bar{x}^{k}\right)=k \mathcal{A}_{H}(\bar{x})$. Hence, there exists a unique integer $k_{l}>1$ such that

$$
\mathcal{A}_{H^{\left(k_{l}\right)}}\left(\bar{y}^{k_{l}} \#(l A)\right) \leq \mathcal{A}_{H^{\left(k_{l}\right)}}\left(\bar{x}^{k_{l}}\right) \text {, but } \mathcal{A}_{H^{\left(k_{l}-1\right)}}\left(\bar{y}^{k_{l}-1} \#(l A)\right)>\mathcal{A}_{H^{\left(k_{l}-1\right)}}\left(\bar{x}^{k_{l}-1}\right) .
$$

A straightforward calculation yields that

$$
\frac{k_{l}-1}{l}<\frac{\lambda_{0}}{\mathcal{A}_{H}(\bar{x})-\mathcal{A}_{H}(\bar{y})} \leq \frac{k_{l}}{l}
$$

and hence

$$
\lim _{l \rightarrow \infty} \frac{k_{l}}{l}=\frac{\lambda_{0}}{\mathcal{A}_{H}(\bar{x})-\mathcal{A}_{H}(\bar{y})} .
$$

Due to (6-12), $k_{l}$ can be equivalently characterized by the requirement

$$
\mu_{H^{\left(k_{l}\right)}}\left(\bar{y}^{k_{l}} \#(l A)\right) \leq \mu_{H^{\left(k_{l}\right)}}\left(\bar{x}^{k_{l}}\right) \text {, but } \mu_{H^{\left(k_{l}-1\right)}}\left(\bar{y}^{k_{l}-1} \#(l A)\right)>\mu_{H^{\left(k_{l}-1\right)}}\left(\bar{x}^{k_{l}-1}\right) \text {. }
$$

Again, by a straightforward calculation, we have

and

$$
\begin{gathered}
\frac{\mu_{H^{\left(k_{l}-1\right)}}\left(\bar{y}^{k_{l}-1}\right)}{l}+2(n+1)>\frac{\mu_{H^{\left(k_{l}-1\right)}}\left(\bar{x}^{k_{l}-1}\right)}{l} \\
\frac{\mu_{H^{\left(k_{l}\right)}}\left(\bar{y}^{k_{l}}\right)}{l}+2(n+1) \leq \frac{\mu_{H^{\left(k_{l}\right)}}\left(\bar{x}^{k_{l}}\right)}{l} .
\end{gathered}
$$

Letting $l \rightarrow \infty$ and taking into account (6-14) and (6-15), we obtain

$$
\frac{\Delta_{H}(\bar{y}) \lambda_{0}}{\mathcal{A}_{H}(\bar{x})-\mathcal{A}_{H}(\bar{y})}+2(n+1)=\frac{\Delta_{H}(\bar{x}) \lambda_{0}}{\mathcal{A}_{H}(\bar{x})-\mathcal{A}_{H}(\bar{y})} .
$$

Since $\lambda_{0}=2(n+1) \lambda$, this readily implies the required equality

$$
\mathcal{A}_{H}(\bar{x})-\lambda \Delta_{H}(\bar{x})=\mathcal{A}_{H}(\bar{y})-\lambda \Delta_{H}(\bar{y}),
$$

concluding the proof of the theorem.

\section{References}

[1] P Albers, A note on local Floer homology arXiv:math.SG/0606600

[2] M Audin, J Lafontaine, editors, Holomorphic curves in symplectic geometry, Progress in Math. 117, Birkhäuser Verlag, Basel (1994) MR1274923

[3] J-F Barraud, O Cornea, Lagrangian intersections and the Serre spectral sequence, Ann. of Math. (2) 166 (2007) 657-722 MR2373371 
[4] P Biran, L Polterovich, D Salamon, Propagation in Hamiltonian dynamics and relative symplectic homology, Duke Math. J. 119 (2003) 65-118 MR1991647

[5] Y V Chekanov, Hofer's symplectic energy and Lagrangian intersections, from: "Contact and symplectic geometry (Cambridge, 1994)", (C B Thomas, editor), Publ. Newton Inst. 8, Cambridge Univ. Press (1996) 296-306 MR1432467

[6] K Cieliebak, V L Ginzburg, E Kerman, Symplectic homology and periodic orbits near symplectic submanifolds, Comment. Math. Helv. 79 (2004) 554-581 MR2081726

[7] C C Conley, Lecture, University of Wisconsin (1984)

[8] M Entov, K-area, Hofer metric and geometry of conjugacy classes in Lie groups, Invent. Math. 146 (2001) 93-141 MR1859019

[9] M Entov, L Polterovich, Rigid subsets of symplectic manifolds arXiv:0704.0105

[10] M Entov, L Polterovich, Calabi quasimorphism and quantum homology, Int. Math. Res. Not. (2003) 1635-1676 MR1979584

[11] A Floer, Morse theory for Lagrangian intersections, J. Differential Geom. 28 (1988) 513-547 MR965228

[12] A Floer, The unregularized gradient flow of the symplectic action, Comm. Pure Appl. Math. 41 (1988) 775-813 MR948771

[13] A Floer, Cuplength estimates on Lagrangian intersections, Comm. Pure Appl. Math. 42 (1989) 335-356 MR990135

[14] A Floer, Symplectic fixed points and holomorphic spheres, Comm. Math. Phys. 120 (1989) 575-611 MR987770

[15] A Floer, Witten's complex and infinite-dimensional Morse theory, J. Differential Geom. 30 (1989) 207-221 MR1001276

[16] B Fortune, A symplectic fixed point theorem for $\mathbf{C P}^{n}$, Invent. Math. 81 (1985) 29-46 MR796189

[17] B Fortune, A Weinstein, A symplectic fixed point theorem for complex projective spaces, Bull. Amer. Math. Soc. (N.S.) 12 (1985) 128-130 MR766969

[18] J Franks, M Handel, Periodic points of Hamiltonian surface diffeomorphisms, Geom. Topol. 7 (2003) 713-756 MR2026545

[19] U Frauenfelder, F Schlenk, Hamiltonian dynamics on convex symplectic manifolds, Israel J. Math. 159 (2007) 1-56 MR2342472

[20] V L Ginzburg, The Conley conjecture arXiv:math.SG/0610956

[21] V L Ginzburg, The Weinstein conjecture and theorems of nearby and almost existence, from: "The breadth of symplectic and Poisson geometry", (JE Marsden, T S Ratiu, editors), Progr. Math. 232, Birkhäuser, Boston (2005) 139-172 MR2103006

[22] V L Ginzburg, Coisotropic intersections, Duke Math. J. 140 (2007) 111-163 MR2355069 
[23] V L Ginzburg, B Z Gürel, Generic existence of infinitely many periodic orbits in Hamiltonian dynamics, in preparation

[24] V L Ginzburg, BZ Gürel, Local Floer homology and the action gap arXiv: 0709.4077v2

[25] V L Ginzburg, B Z Gürel, Periodic orbits of twisted geodesic flows and the WeinsteinMoser theorem, to appear in Comment. Math. Helv. arXiv:0705.1818

[26] V L Ginzburg, B Z Gürel, Relative Hofer-Zehnder capacity and periodic orbits in twisted cotangent bundles, Duke Math. J. 123 (2004) 1-47 MR2060021

[27] V L Ginzburg, E Kerman, Homological resonances for Hamiltonian diffeomorphisms and Reeb flows, Int. Math. Res. Not. (2009) Art. ID rnp 120, 16 pp

[28] V Guillemin, VL Ginzburg, Y Karshon, Moment maps, cobordisms, and Hamiltonian group actions, Math. Surveys and Monogr. 98, Amer. Math. Soc. (2002) MR1929136 Appendix J by M Braverman

[29] B Z Gürel, Totally non-coisotropic displacement and its applications to Hamiltonian dynamics, Commun. Contemp. Math. 10 (2008) 1103-1128 MR2483254

[30] N Hingston, Subharmonic solutions of Hamiltonian equations on tori, to appear in Ann. of Math. Available at http://comet.lehman.cuny.edu/sormani/others/ hingston.html

[31] H Hofer, Lusternik-Schnirelman-theory for Lagrangian intersections, Ann. Inst. H. Poincaré Anal. Non Linéaire 5 (1988) 465-499 MR970850

[32] H Hofer, D A Salamon, Floer homology and Novikov rings, from: "The Floer memorial volume", (H Hofer, CH Taubes, A Weinstein, E Zehnder, editors), Progr. Math. 133, Birkhäuser, Basel (1995) 483-524 MR1362838

[33] H Hofer, C Viterbo, The Weinstein conjecture in the presence of holomorphic spheres, Comm. Pure Appl. Math. 45 (1992) 583-622 MR1162367

[34] H Hofer, E Zehnder, Symplectic invariants and Hamiltonian dynamics, Birkhäuser Advanced Texts: Basler Lehrbücher, Birkhäuser Verlag, Basel (1994) MR1306732

[35] E Kerman, Squeezing in Floer theory and refined Hofer-Zehnder capacities of sets near symplectic submanifolds, Geom. Topol. 9 (2005) 1775-1834 MR2175157

[36] E Kerman, Hofer's geometry and Floer theory under the quantum limit, Int. Math. Res. Not. (2008) Art. ID rnm 137, 36pp MR2417790

[37] H V Lê, K Ono, Cup-length estimates for symplectic fixed points, from: "Contact and symplectic geometry (Cambridge, 1994)", (C B Thomas, editor), Publ. Newton Inst. 8, Cambridge Univ. Press (1996) 268-295 MR1432466

[38] G Liu, G Tian, Weinstein conjecture and GW-invariants, Commun. Contemp. Math. 2 (2000) 405-459 MR1806943 
[39] G Lu, Gromov-Witten invariants and pseudo symplectic capacities, Israel J. Math. 156 (2006) 1-63 MR2282367

[40] D McDuff, Hamiltonian $S^{1}$-manifolds are uniruled, Duke Math. J. 146 (2009) 449507 MR2484280

[41] D McDuff, D Salamon, Introduction to symplectic topology, Oxford Math. Monogr., Oxford Science Publ., The Clarendon Press, Oxford Univ. Press, New York (1995) MR1373431

[42] D McDuff, D Salamon, J-holomorphic curves and symplectic topology, Amer. Math. Soc. Coll. Publ. 52, Amer. Math. Soc. (2004) MR2045629

[43] Y-G Oh, A symplectic fixed point theorem on $T^{2 n} \times \mathbf{C P}^{k}$, Math. Z. 203 (1990) 535-552 MR1044063

[44] Y-G Oh, Construction of spectral invariants of Hamiltonian paths on closed symplectic manifolds, from: "The breadth of symplectic and Poisson geometry", (J E Marsden, T S Ratiu, editors), Progr. Math. 232, Birkhäuser, Boston (2005) 525-570 MR2103018

[45] K Ono, On the Arnold conjecture for weakly monotone symplectic manifolds, Invent. Math. 119 (1995) 519-537 MR1317649

[46] Y Ostrover, Calabi quasi-morphisms for some non-monotone symplectic manifolds, Algebr. Geom. Topol. 6 (2006) 405-434 MR2220683

[47] S Piunikhin, D Salamon, M Schwarz, Symplectic Floer-Donaldson theory and quantum cohomology, from: "Contact and symplectic geometry (Cambridge, 1994)", (C B Thomas, editor), Publ. Newton Inst. 8, Cambridge Univ. Press (1996) 171-200 MR1432464

[48] M Poźniak, Floer homology, Novikov rings and clean intersections, from: "Northern California Symplectic Geometry Seminar”, (Y Eliashberg, D Fuchs, T Ratiu, A Weinstein, editors), Amer. Math. Soc. Transl. Ser. 2 196, Amer. Math. Soc. (1999) 119-181 MR1736217

[49] D A Salamon, Lectures on Floer homology, from: "Symplectic geometry and topology", (Y Eliashberg, L Traynor, editors), IAS/Park City Math. Series 7, Amer. Math. Soc. (1999) xiv+430 MR1702940

[50] D A Salamon, E Zehnder, Morse theory for periodic solutions of Hamiltonian systems and the Maslov index, Comm. Pure Appl. Math. 45 (1992) 1303-1360 MR1181727

[51] M Schwarz, A quantum cup-length estimate for symplectic fixed points, Invent. Math. 133 (1998) 353-397 MR1632778

[52] M Schwarz, On the action spectrum for closed symplectically aspherical manifolds, Pacific J. Math. 193 (2000) 419-461 MR1755825

[53] A Cannas da Silva, Lectures on symplectic geometry, Lecture Notes in Math. 1764, Springer, Berlin (2001) MR1853077 
[54] M Usher, Spectral numbers in Floer theories, Compos. Math. 144 (2008) 1581-1592 MR2474322

[55] M Usher, Floer homology in disk bundles and symplectically twisted geodesic flows, J. Mod. Dyn. 3 (2009) 61-101 MR2481333

[56] C Viterbo, Symplectic topology as the geometry of generating functions, Math. Ann. 292 (1992) 685-710 MR1157321

[57] C Viterbo, Functors and computations in Floer homology with applications. I, Geom. Funct. Anal. 9 (1999) 985-1033 MR1726235

Department of Mathematics, UC Santa Cruz

Santa Cruz, CA 95064, USA

Department of Mathematics, Vanderbilt University

Nashville, TN 37240, USA

ginzburg@math.ucsc.edu, basak.gurel@vanderbilt.edu

http://count.ucsc.edu/ ginzburg/,

http://www. math.vanderbilt.edu/ gurelzb/

Proposed: Yasha Eliashberg

Seconded: Leonid Polterovich, Danny Calegari

Received: 5 January 2009 Accepted: 29 June 2009 